\title{
Integrated Indium Micromirrors for Optofluidics 2013
}

\author{
Rafael Gomez Bule
}




\section{Table of Contents}

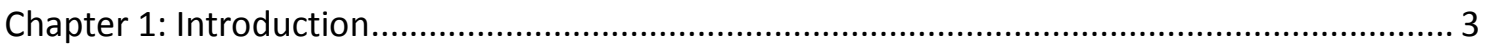

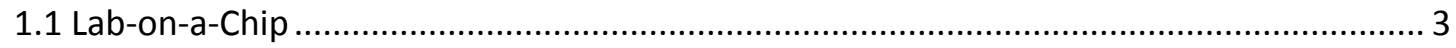

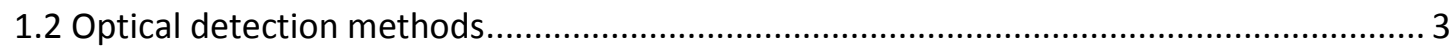

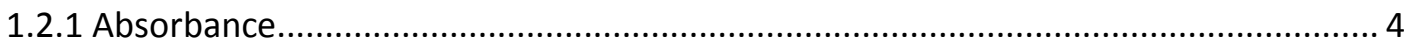

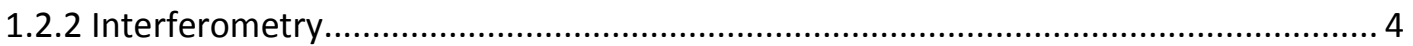

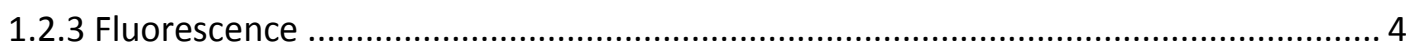

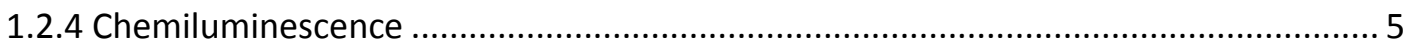

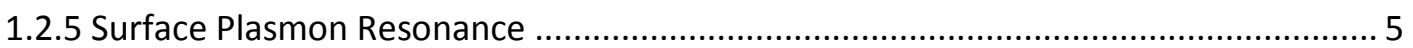

1.3 Selection of absorbance measurements ...................................................................... 5

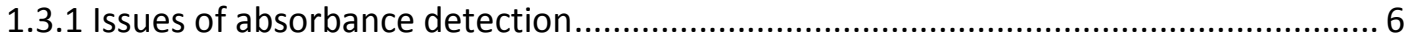

1.4 Previous research on improving absorbance measurements........................................... 7

1.5 Kinds of micromirrors that have been developed ......................................................... 10

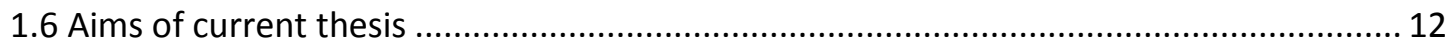

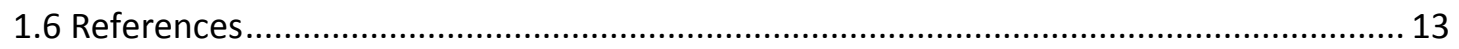

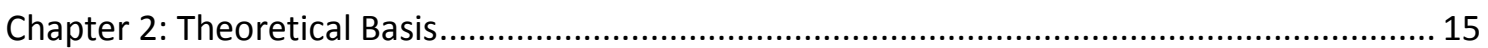

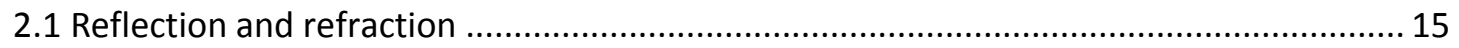

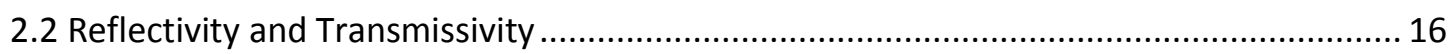

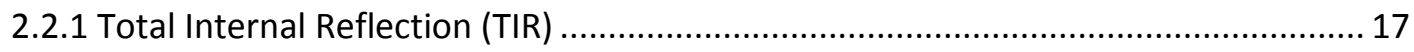

2.3 Optical properties of metals....................................................................................... 17

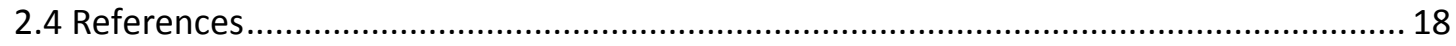

Chapter 3: Design and Theoretical Analysis of the Device....................................................... 19

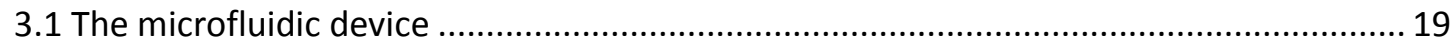

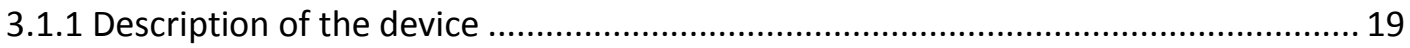

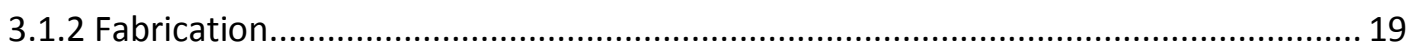

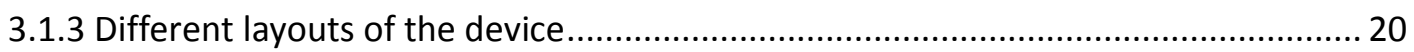

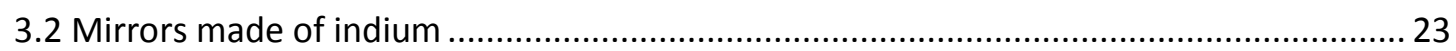

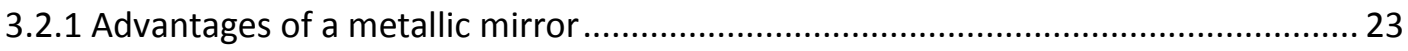

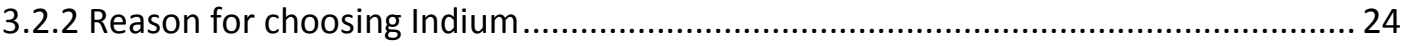

3.2.3 Theoretical reflectivity of the indium micromirrors .................................................. 25

3.3 Measurement Configuration ................................................................................. 30

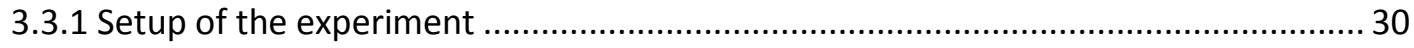

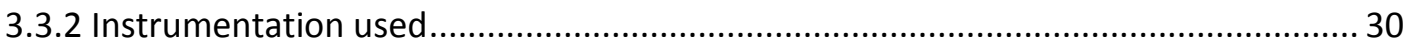

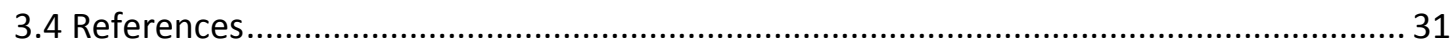




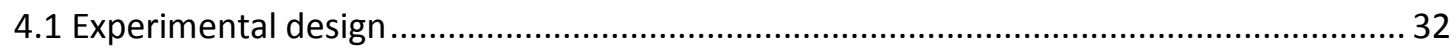

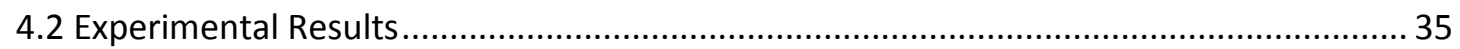

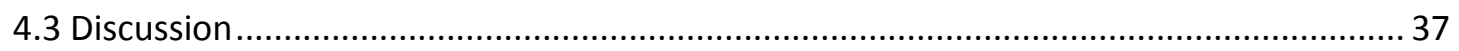

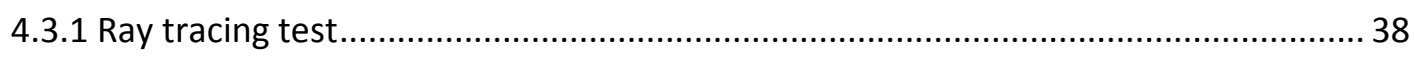

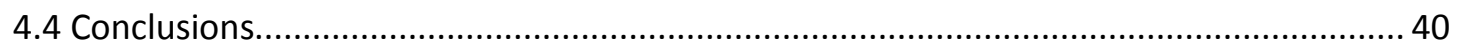

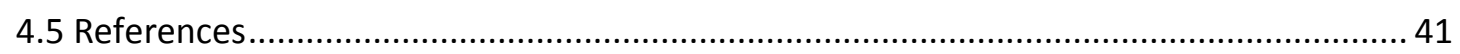

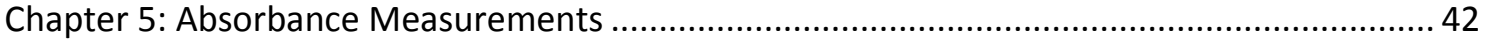

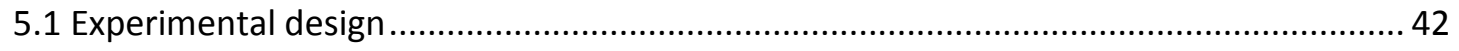

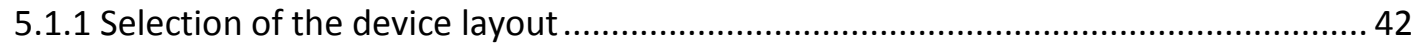

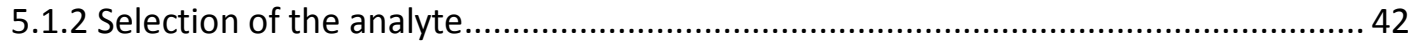

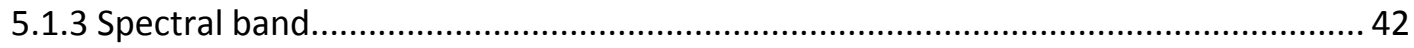

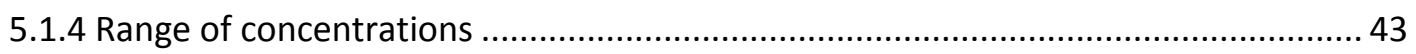

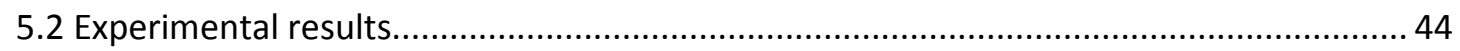

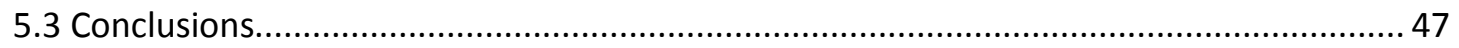

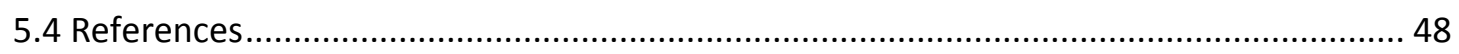

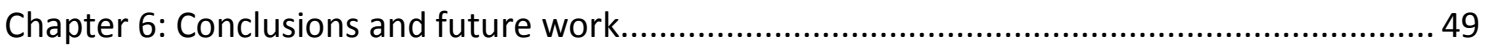

6.1 Conclusions on indium micromirrors ............................................................................... 49

6.2 Conclusions on absorbance measurements................................................................... 49

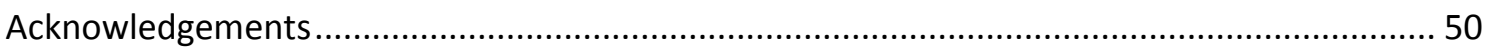




\section{Chapter 1: Introduction}

\subsection{Lab-on-a-Chip}

Lab-on-a-chip (LOC) devices make possible to perform one or some laboratory functions and processes in a miniaturized chip format. Mostly, LOC devices are used in biomedical assays and point-of-care diagnostics (POC), whose applications include rapid pathogen detection, clinical and forensic diagnosis, blood chemistry and DNA analysis, electrophoresis, etc.

LOC devices, are not also intended to miniaturize the size of the instrumentation but also the amount of analyte to perform a measurement. That is why they often have to deal with performing good quality measurements with a few microliters or even picoliters of the sampling fluid. This gives place to what is called microfluidics.

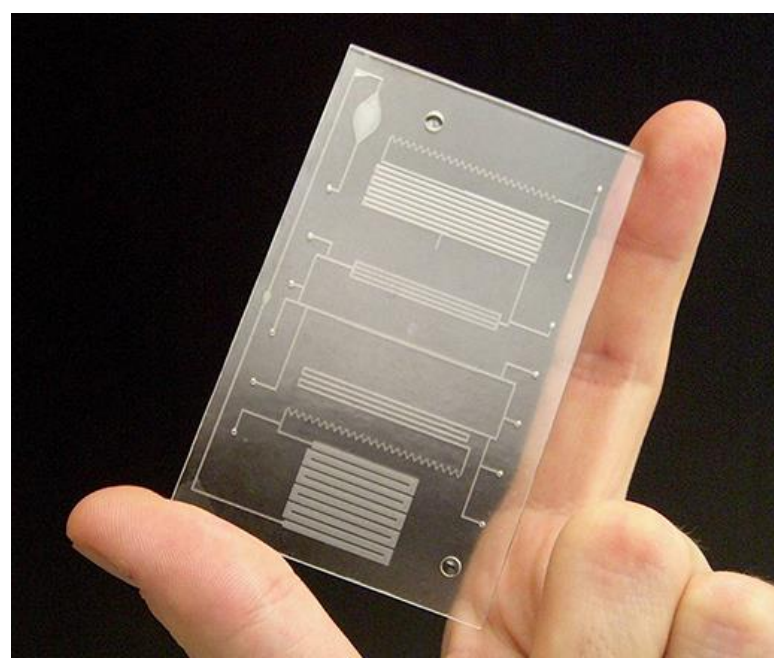

Fig. 1.1 Lab-on-a-chip Source:http://www.fhcmi.org/LSE/includes/assets/ images/Project-08b/lab-on-a-chip-in-hand.jpg

There are many kinds of materials to fabricate LOC devices, including silicon, glass, or several types of polymers -being polydimethylsiloxane (PDMS) the most commonly used-. Also a broad variety of fabrication techniques are being used, but the most usual is photolithography, due to its good results in miniaturizing precise layouts[1].

\subsection{Optical detection methods}

Optical detection methods applied to microfluidics have the advantages of both high accuracy and cost efficiency, since there is no need of electrodes to be used and optical devices are cheaper and give high quality and precise results. Furthermore, using light to perform measurements in biomedical applications has the advantage of being harmless and less invasive for cells in in-vivo measurements as long as the correct wavelength is used. 


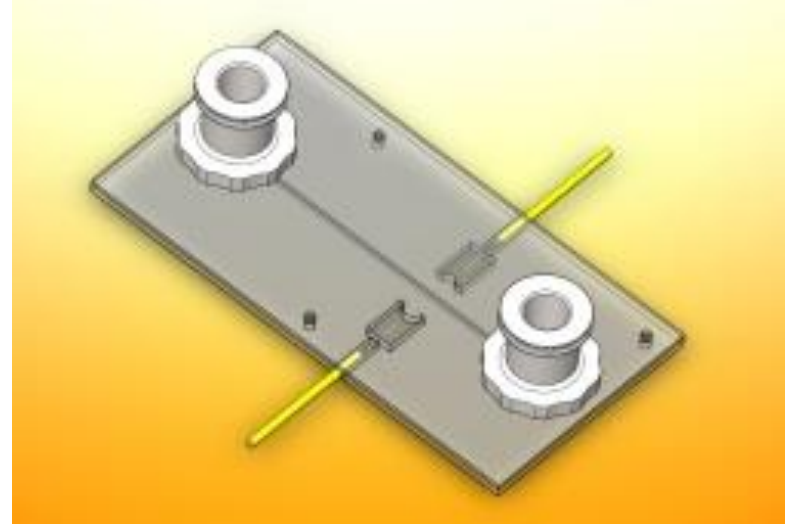

Fig. 1.2 Optical detection microfluidic device Source: http://www.translume.com/images/IntFib.JPG

Herein we present and describe the most common optical detection methods that are currently being used in microfluidic biosensors, which are: Absorbance, interferometry, fluorescence, chemiluminescence and surface plasmon resonance[2]. The most important handicap on measuring these parameters at the scale of microfluidics is the short optical path length, which limits the sensitivity of the device. To solve this issue many components such as mirrors, lenses or filters are currently being developed for microfluidic biosensors, giving place to actual LOCs.

\subsubsection{Absorbance}

Absorbance measures the logarithmic ratio between the incident and transmitted radiation upon a material. In spectroscopy, this parameter, measured as a function of wavelength, is really useful, revealing peaks of absorption at certain frequencies that can help to identify the composition and concentration of the studied substance[3]. It is calculated by the following formula, being ' $\mathrm{A}$ ' the absorbance, ' $\mathrm{I}_{0}$ ' the reference intensity -i.e. the intensity received from a zero concentration reference sample- and ' $\mathrm{l}$ ' the received intensity:

$$
A=\log _{10} \frac{I_{0}}{I}
$$

Eq. 1.1 Absorbance formula. A: Absorbance; $\mathrm{I}_{0}$ : reference intensity; I: received intensity

\subsubsection{Interferometry}

Interferometry refers to a family of techniques that make use of the concept of superposition to combine identical signals with different optical paths and extract data and conclusions from the resulting phase shift[4].

\subsubsection{Fluorescence}

Fluorescence is an optical method that measures the emission of light by a substance that has absorbed some kind of electromagnetic radiation, including light itself. Usually, if the absorbed radiation is not too intense, the emitted light has a longer wavelength (lower energy), thus allowing to be filtered and measured. 


\subsubsection{Chemiluminescence}

Chemilumenescence measures the emission of light as a result of a chemical reaction[5]. These methods have the particularity that no excitation instrumentation is needed.

\subsubsection{Surface Plasmon Resonance}

Surface plasmon resonance (SPR) detection allows observing the binding events between an analyte and an immobilized biomolecule by measuring the refractive index change at a metal surface functionalized with probe molecules. As the resonance frequency of a thin metal layer is highly sensitive to any variation at its boundary, when it is radiated with light, the presence of a sample at the other side may cause a shifting of this frequency. This will allow measuring a change in the refractive index[6].

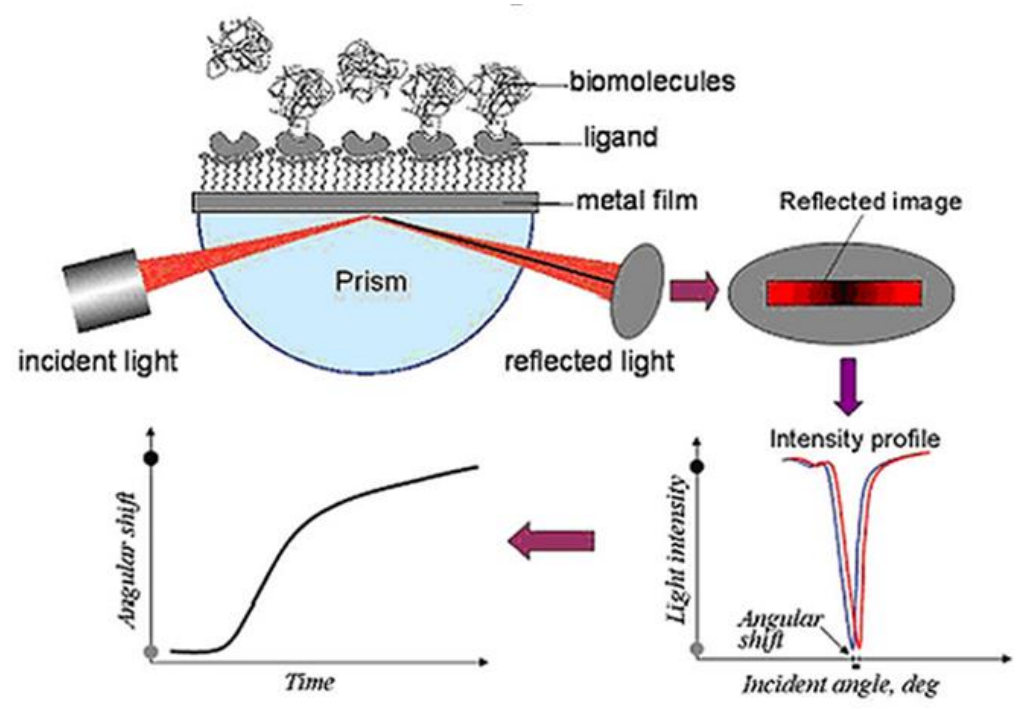

Fig. 1.3 Surface plasmon resonance detection Source: http://www.biosensingusa.com/images/application101_fig2.png

\subsection{Selection of absorbance measurements}

In this thesis we are going to develop and present an optical detection microfluidic device that meets the following approaches:

- Monolithic integration: In microfluidics typically soft lithography makes it possible, since devices are casted on a master and fabricated in one single step.

- Inexpensive fabrication: Again, soft lithography helps with this aim, since one single master allows replicating as many devices as wanted. In addition, the fact that elastomeric materials -most notably PDMS- and optical fibers are relatively cheap also favors an economic fabrication process.

- Simple and ubiquitous measuring process: Further from just taking measurements in an academic or research environment, lab-on-chips are intended to be a portable lab.

Among all the methods described in Section 1.2, fluorescence detection is the most sensitive optical method [7], allowing single molecule detection in some circumstances[8]. However it has the disadvantage that most molecules do not naturally fluoresce, thus requiring the introduction of a labeling step before any separation, which requires sample manipulation, which may introduce interferences in the measurements. 
Furthermore, when an excited fluorescent molecule relaxes to its ground energy level, the direction of the light emitted photon is completely random, regardless the direction of the exciting wave. This randomness is what would make most of the emitted light not to be detected by a simple optical fiber, but only those photons directed towards it. Thus, if a better quality measurement is intended, the device design gets more complex and expensive, having to involve photosensitive materials and surfaces.

Chemiluminescence has the same issues than fluorescence as regards the percentage of the emitted light that can be detected with simple reception instrumentation, since chemically excited molecules emit light also isotropically. Besides, once the sample is mixed with the chemical catalyzer, it is contaminated and no further measurements can be taken.

Surface plasmon resonance measurements need a very concrete structure which is not easy to implement with a low cost and simple fabrication process. Same thing happens to interferometry, which requires the integration of beam splitters and other path-tracing elements.

As for optical detection based on the measurement of absorbance, it is remarkable because of its simple yet sensitive operation. In order to measure this parameter we only need to inject light at one side of the sample and collect it at the other side, and very simple calculations are needed to get the actual absorbance value and the final concentration of the sample (equations 1.1 and 1.2). Due to this simplicity, a basic design to measure this parameter does not involve too complex or too many fabrication steps, allowing us to focus in improving the quality of the measurements.

After the discussion presented above, seems that the most suitable optical detection method for our purposes -set at the beginning of this section- is absorbance. That is why, among the five parameters described in Section 1.2, we will present a proposal for a microfluidic device intended for absorbance detection using an approach that is fast, simple and inexpensive.

\subsubsection{Issues of absorbance detection}

One of the major problems on absorbance detection at the scale of microfluidics is the shortness of the optical path length. The shorter it is the higher will be the limit of detection (LOD) -i.e. the minimum amount of concentration that can be sensed-. This means that, shortening the optical path through the analyte we will need more concentration of it to be detected by the device.

When dealing with microfluidics, the sample to analyze is scarce, and a good instrument will be the one that can make precise measurements (high sensitivity) with a lower concentration of analyte -i.e. low LOD[9]-. This can be achieved by increasing the optical path length through our scarce sample, as is stated by the Beer-Lambert law[3]. This law relates the absorbance of light to the properties of the material within it propagates, being the length one of these properties (see Equation 1.2).

$$
A=\varepsilon L c
$$


Eq. 1.2 Beer-Lambert law. A: Absorbance; $\varepsilon$ : molar absorbtivity with units of $\mathrm{L} \mathrm{mol}^{-1} \mathrm{~cm}^{-1}$; L: Optical path length; c: Analyte concentration in $\mathrm{mol} \mathrm{L}^{-1}$.

As can be seen, a very short optical path would lead to such a low absorbance reading that the instrument might not be able to detect. However, enlarging the optical path would increase the light absorbed, making it possible to be measured by the instrument without having increased the sample concentration.

Both the absorbance and the molar absorbtibity are wavelength dependent, so the spectrum of interest will be a plot of either ' $A$ ' or ' $E$ ' as a function of wavelength.

\subsection{Previous research on improving absorbance measurements}

Many methods have been proved effective and successful for increasing the sensitivity of absorbance measurements in very low concentrations of analyte, most of them using reflection and light guidance methods that increase the sensitivity of the system. However, very few satisfy all the requirements of good sensitivity, low sample amount, ubiquity and monolithic integration.

\section{Tinted substrate absorption cell}

There are research groups studying the possibility of minimizing the effects of extern noise interferences and light diffraction by building a micro-channel inside a tinted substrate[10] (see Figure 1.4). Thus, the substrate absorbs extern light as well as stray light from the source that might interfere in the measure, creating a pin-hole configuration over the entire length of the absorption cell. This enables long path lengths with an excellent signal to noise ratio and a higher sensitivity.

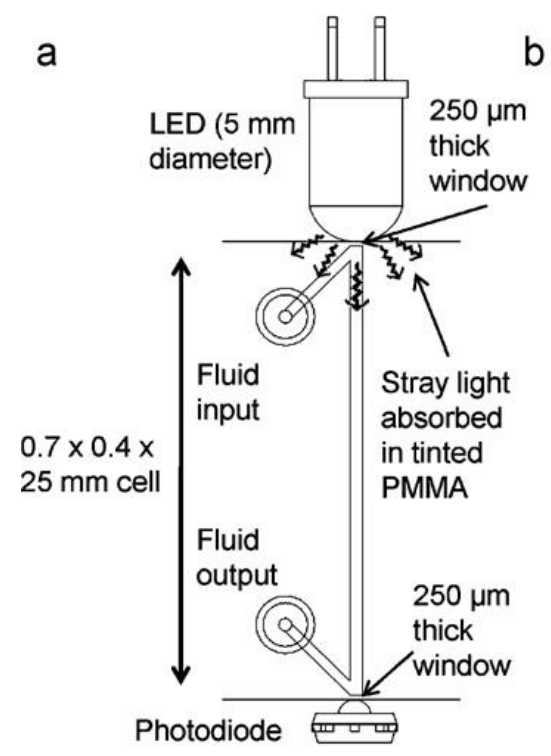

Fig. 1.4 Schematic of a tinted substrate absorption cell. Source: [10] 


\section{Cavity Ringdown Spectroscopy}

One method commonly used to enlarge the optical path in absorbance spectroscopy for gases at macroscopic scales (cavities in the order of meters) is Cavitiy Ringdown Spectroscopy (CRDS). However, some research groups have brought this concept to the area of microfluidics, as the high sensitivity and low LOD of these techniques make them an attractive option, heavily increasing the optical path length without increasing the sample volume. CRDS is a highly sensitive absorption technique based on the decay of light intensity inside an optical cavity. This method uses the comparison between the decay time constants -or ringdown times- measured in the absence and presence of the analyte to calculate the absorption of the sample.

Most attempts to merge CRDS with microfluidics have employed Fiber-loop cavities (FLRDS) [11], which confine light using an optical fiber loop and leaving a micro-gap between the fiber ends for the analyte (see Figure 1.5). Once a light pulse is coupled into the system by a fiber splice connector it performs many round trips before the intensity is below the detection threshold. The ringdown time difference between measures with or without the sample lets calculating the absorption of the analyte.

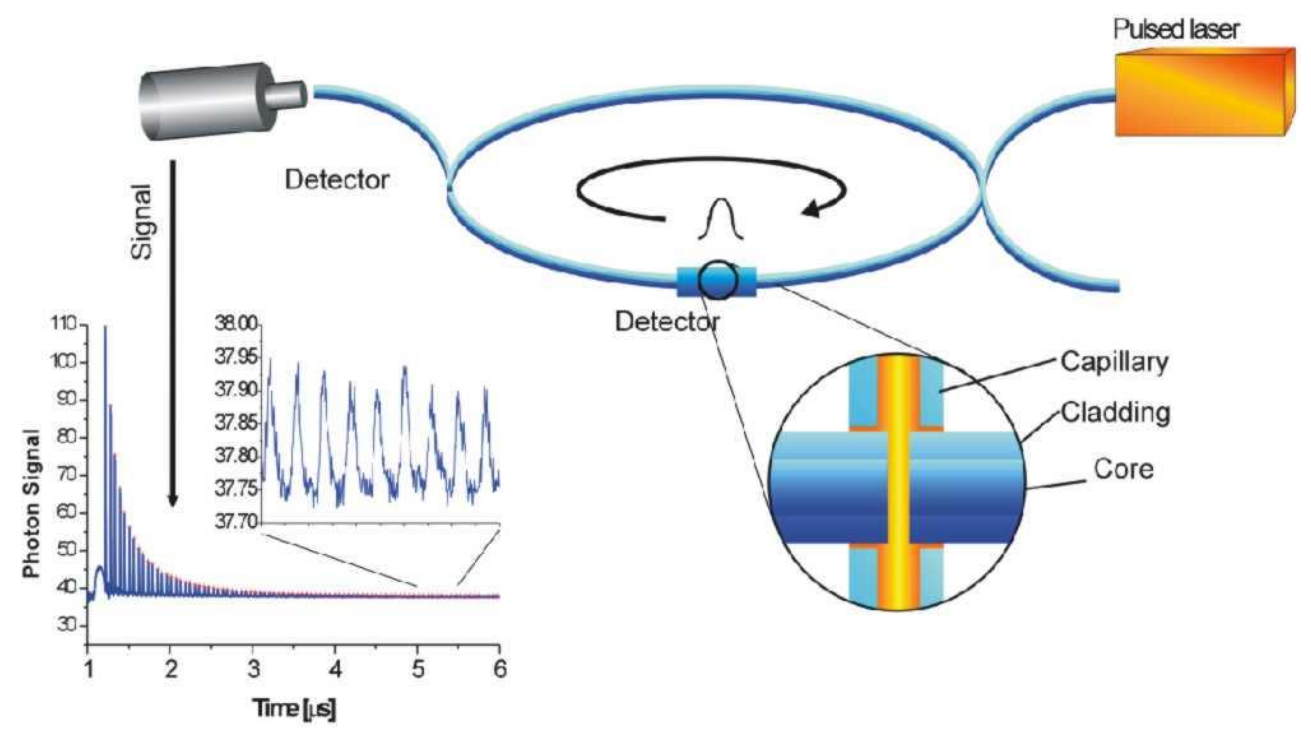

Fig. 1.5 Fiber-loop CRDS system diagram.

Source: http://www.chem.queensu.ca/people/faculty/loock/pulsed\%20FLRDS\%20setup.jpg

Nevertheless there have been groups that have also succeeded to perform a Fabry-Perot cavity for CRDS (the same kind used in first macroscopic models) using two micromirrors [12]. This has the advantage of a lower loss than FLRDS, achieving higher sensitivities. 
(a)

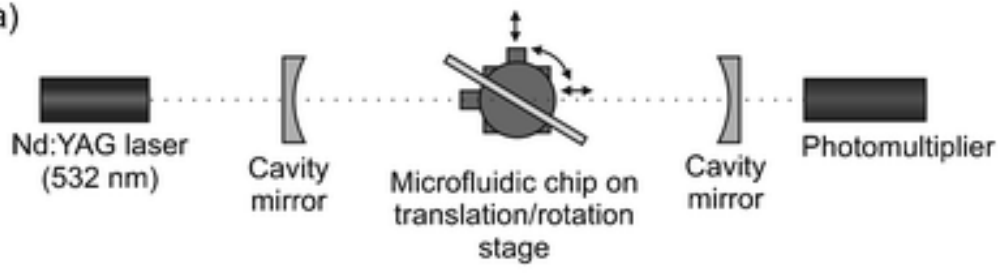

(b)

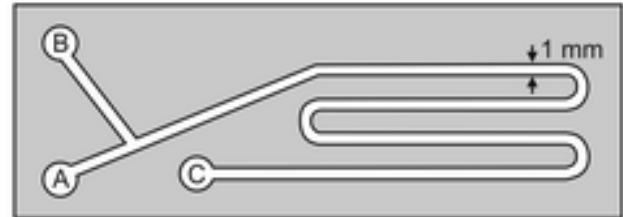

Fig. 1.6 (a) Schematic of the experimental setup of Fabry-Perot CRDS; (b) design of the microfluidic chip used in this example.

Source: [12]

Both of these CRDS methods are high quality efficient measures, which makes them of great interest. However, the two of them are not a monolithic solution, which affects the fabrication cost, the calibration issues and the ubiquity of the system.

\section{Embedded micro mirrors}

This might be the closest approach to what this thesis tries to achieve, being probably a further step for the evolution of an itinerary that has been settled mainly by the research group of Doctor A. Llobera. He developed and studied three different methods to measure absorbance in a microfluidic PDMS lab-on-a-chip cell (Figure 1.7). His contributions are based into taking profit of the internal reflection of the emitted light beam into the microfluidic cavity in order to increase the optical path length. Each one of the following methods is just one step more to its previous version.

a) PDMS hollow Abbe Prism [13]: A prism shaped microfluidic cavity was designed to take profit of the change of refractive index between air (or the analyte) and the PDMS walls. This would allow redirecting the light beam back to the cavity and thus, increment the optical path length. However, although the sample refractive index is usually unknown, design was made supposing a buffer solution ( $n=1.334)$. As the refractive index of PDMS $(n=1.41)$ is higher than the substance's, much of the light escapes when hitting the prism wall, and only a fraction of it is redirected.

b) PDMS hollow prism with air mirror [14]: In order to improve the results obtained in the Abbe prism, an air hollow mirror was placed after the PDMS wall. Thus, as air refractive index is lower than PDMS's, total internal reflection can be achieved, which introduced a great improvement in sensitivity and LOD. However, due to light dispersion, not all the light could be coupled back into the reception optical fiber.

c) Multiple internal reflection (MIR) systems using air concave mirrors[15]: Regarding the successful results of the previous device, Dr. A. Llobera's research group decided to take profit of the new concept of air micromirrors in order to achieve MIR and make the optical path even longer. That was made by introducing several mirrors along an outstretched micro-channel. Furthermore, the mirrors were designed with a concave shape to re-focus the light in order to solve the problem of dispersion. 
(a)

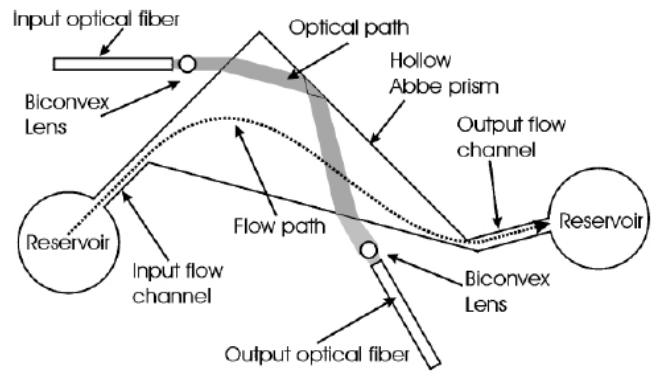

(b)

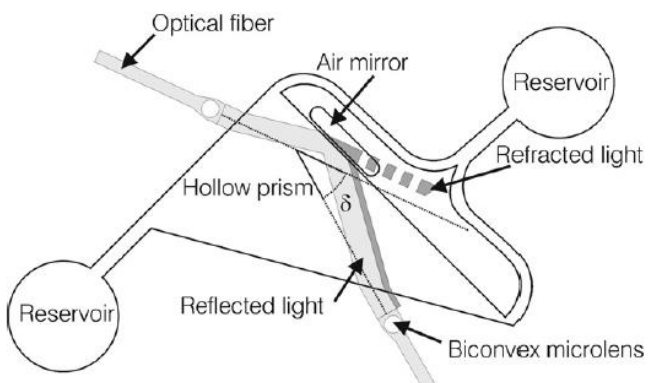

(c)

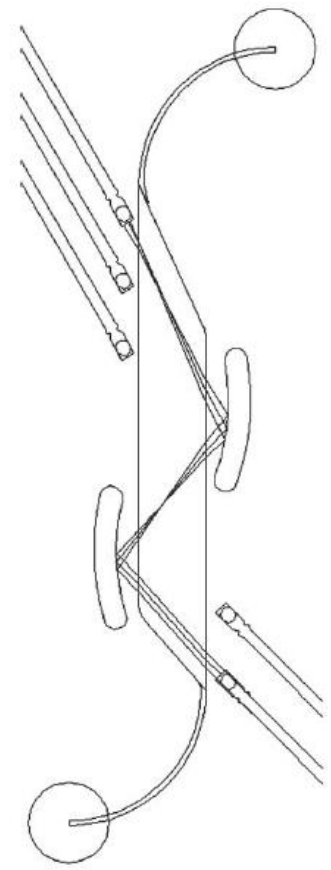

Fig. 1.7 (a) PDMS hollow Abbe Prism; (b) PDMS hollow prism with air mirror; (c) MIR system with air concave mirrors.

Source: [13-15]

As it can be observed, this latest improvement leads to a single-step fabricated portable device that can perform high-sensitivity measurements with low amount of analyte. That is, a device that meets the already set up requirements of monolithic integration, ubiquity, cheap fabrication and few sample needs. It seems then, that embedded micromirrors in a PDMS cell are a good way to achieve the objectives of this thesis. The next step will then be to take a look at the micromirrors already developed to see if any of them might be suitable to replace these air ones.

\subsection{Kinds of micromirrors that have been developed}

So far, there has been stated that a longer optical path would improve the absorbance measurements. However, there is also an interest in doing it without increasing the amount of sample under study. One way to achieve this enlargement of the optical path without increasing the analyte amount is using micromirrors.

Micromirrors are used to bounce the light beam back into the sample once it has passed all the way through, thus doubling or multiplying (depending on the number of mirrors implemented) the optical path length.

Herein we review some of the already developed micromirrors, examining their possibilities to be embedded in a PDMS absorption cell chip.

\section{Adjustable micro-concave mirror for bio-detection applications}

A research group of the National University of Formosa (Taiwan) has developed an adjustable micro-concave mirror to perform optical bio-detection applications[16]. This mirror is built in a 
polysilicon thin film over a silicon substrate, and has a thin gold film over it to increase reflectivity. Due to the properties of the polysilicon, its shape (focal length) can be adjusted by the voltage applied to an electrode located just under the mirror, and also by controlled temperature changes. The diameter achieved for this mirror was $800 \mu \mathrm{m}$.

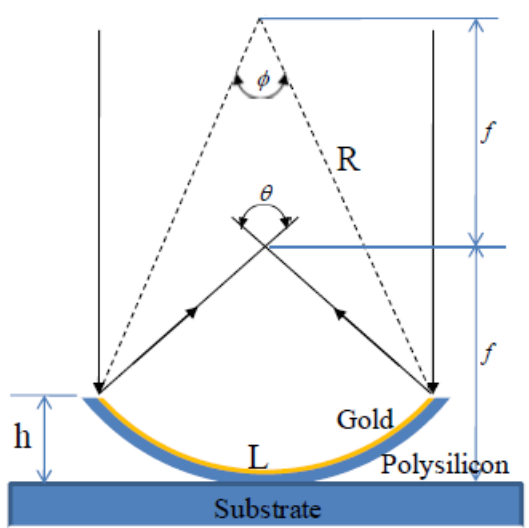

(a)

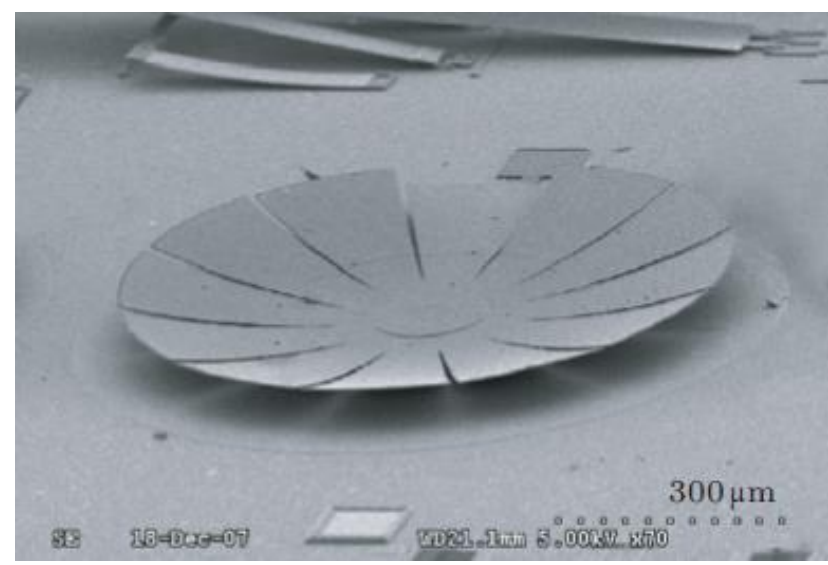

(b)

Fig. 1.8 Schematic composition (a) and picture (b) of the micromirror. Source: [16]

The micromirror was designed for bio-detection applications, especially for fluorescence. More than to bounce light beams, it was intended to focus them into one point. For absorbance detection, where the function of the mirrors would be also enlarging the path length by one or several reflections, these components may not be suitable.

Thus, as regards absorbance detection, this mirror would not be the best option. First, if it was intended to be embedded into a microfluidic device, it results to be too large. A diameter of $800 \mu \mathrm{m}$ is too much for a soft-lithography-built chip. Furthermore, the incorporation of this mirror to an absorbance detection system, either embedded or out of the microfluidic chip, would not result into a desired monolithic approach.

\section{Silicon micromirrors}

There have been also attempts to build concave micromirrors in silicon by ion beam irradiation of a wafer masked with photoresist material [17]. Although silicon is useful for integrating light beam guidance into micro-electro-mechanical-systems (MEMS), it might not be the best option for improving optical detection in a microfluidic chip. As regards size, since down to $20 \mu \mathrm{m}$ diameter mirrors can be achieved, it would not be a limiting factor. However, the integration of the micromirror into the chip would not be that easy. In addition, a mirror fabrication step would be added to the microfluidic cell fabrication process, thus affecting the monolithic aim.

\section{Elastomeric mirrors}

A research group of Harvard University developed in 1996 a method for fabricating deformable elastomeric optical components, including micromirrors among them [18]. They casted PDMS 
against a glass lens and after removing the master lens either gallium or mercury was poured into its place. Then they sealed it with a second layer of PDMS (Figure 1.9).

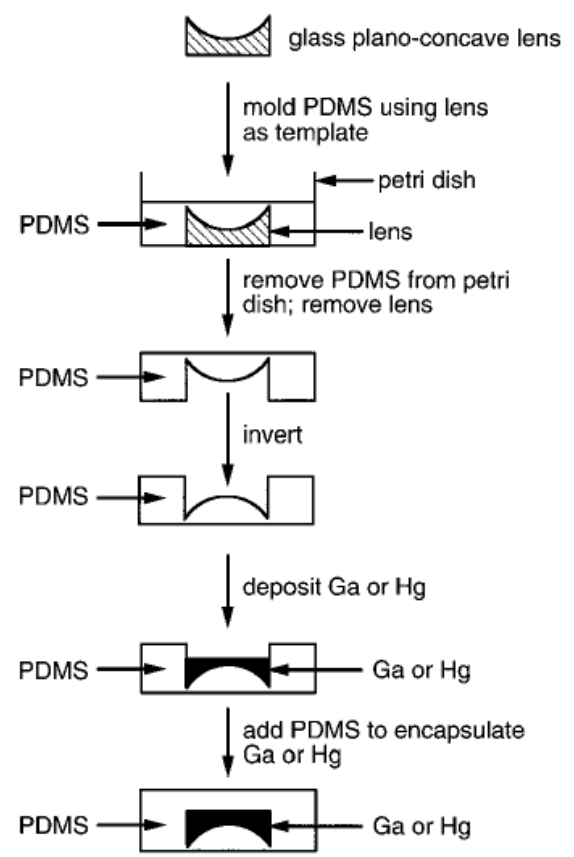

Fig. 1.9 Schematic of the elastomeric mirror fabrication process.

Source: [18]

This idea could be adapted and this kind of mirrors could be implemented into a chip similar to Dr. Llobera's last improvement [15], filling the air mirrors with $\mathrm{Hg}$ or Ga. However, these chips do not need to be deformable. In fact, it is better that they are rigid to make sure the light path is the same always and incident angles are not varied. That is why, if the chip is meant to be rigid, there is no need to use a liquid metal to fill the mirrors, which will complicate the fabrication process and probably compromise the monolithic integration of the system.

\subsection{Aims of current thesis}

After all the research that has been exposed above, we present here our own alternative to improve absorbance measurements, combining elements already existent with some selfdeveloped techniques.

We will inspire the design of our device in the one in [15]. However, in [15] micromirrors are made of air inside a PDMS stamp. These are both considered dielectric materials, which have the advantage of allowing a 100\% reflectance (Total Internal Reflection, TIR) for a determinate range on incidence angles; however reflectivity quickly drops when the incidence angle is out of that range. We want, then, to study the possibilities of embedding metallic mirrors in a PDMS device, since metals -opposite to dielectrics- provide high reflectance even for perpendicularly incident light. The interest on this is due to two main reasons: (i) having more freedom in the angle we input the light in our system we will be able to reach more compact layouts for our devices and (ii) there are also some applications in microfluidics that require for normal incidence [19]. 
Thus, we want to change the air mirrors in [15] by metallic ones and analyze the improvements and consequences of it. We propose in this thesis the integration of metallic (indium) mirrors to microfluidic devices using an approach that is fast, simple, and inexpensive.

\subsection{References}

[1] K. E. Herold and A. Rasooly, Lab-on-a-Chip Technology (Vol. 1): Fabrication and Microfluidics: Caister Academic Press, 2009.

[2] F. B. Myers and L. P. Lee, "Innovations in optical microfluidic technologies for point-ofcare diagnostics," Lab Chip, vol. 8, pp. 2015-2031, 2008.

[3] W. College. (2004). Absorption Spectrophotometry [Online]. Available: http://www.wellesley.edu/Biology/Concepts/Html/absorptionspectro.html

[4] A. Crespi, Y. Gu, B. Ngamsom, H. J. W. M. Hoekstra, C. Dongre, M. Pollnau, R. Ramponi, H. H. v. d. Vlekkert, P. Watts, G. Cerullo, and R. Osellame, "Three-dimensional MachZehnder interferometer in a microfluidic chip for spatially-resolved label-free detection," vol. 10, pp. 1167-1173, 2010.

[5] M. Kamruzzaman, A.-M. Alama, K. M. Kima, S. H. Lee, Y. H. Kimb, G.-M. Kimc, and T. D. Dang, "Microfluidic chip based chemiluminescence detection of L-phenylalanine in pharmaceutical and soft drinks," Food Chemistry, vol. 135, pp. 57-62, 2012.

[6] Biosensing-Instrument-Incorporated. (2006). Surface Plasmon Resonance Technology [Online]. Available:

http://www.biosensingusa.com/biosensing instrument technology.html

[7] Photon-Technology-International. (2005). The Measurement of Sensitivity in Fluorescence Spectroscopy [Online]. Available: http://www.ptinj.com/LaserStrobe/TechNotes/MeasurementSensitivity.pdf

[8] H. Li, X. Ren, L. Ying, S. Balasubramanian, and a. D. Klenerman, "Ultrasensitive coincidence fluorescence detection of single DNA molecules.," Analytical Chemistry, vol. 75, pp. 1664-1670, 2003.

[9] J. L. Rudy, "Differentiating between Sensitivity and Limit of Detection," Clinical Chemistry, vol. 35, p. 509, March 1989.

[10] C. F. A. Floqueta, V. J. Siebena, A. Milania, E. P. Joly, I. R. G. Ogilviea, H. Morganb, and M. C. Mowlema, "Nanomolar detection with high sensitivity microfluidic absorption cells manufactured in tinted PMMA for chemical analysis," Talanta, vol. 84, pp. 235239, 2011.

[11] N. Trefiak, J. Barnes, R. Li, Z. Tong, R. D. Oleschuk, and H.-P. Loock, "Optical Loss Measurements Using Photon Lifetimes in Optical Wave Guides," IEEE Leos Newsletter, 2005.

[12] D. James, B. Oag, C. M. Rushworth, J. W. L. Lee, J. Davies, J. T. Cabral, and C. Vallance, "High-sensitivity online detection for microfluidics via cavity ringdown spectroscopy," RSC Advances, vol. 2, pp. 5376-5384, 2012.

[13] A. Llobera, R. Wilke, and S. Büttgenbach, "Poly(dimethylsiloxane) hollow Abbe prism with microlenses for detection based on absorption and refractive index shift," Lab Chip, vol. 4, pp. 24-27, 2004.

[14] A. Llobera, R. Wilke, and S. Büttgenbach, "Enhancement of the response of poly(dimethylsiloxane) hollow prisms through air mirrors for absorbance-based sensing," Talanta, vol. 75, pp. 473-479, 2008.

[15] A. Llobera, S. Demming, R. Wilke, and S. Büttgenbach, "Multiple internal reflection poly(dimethylsiloxane) systems for optical sensing," Lab Chip, vol. 7, pp. 1560-1566, 2007.

[16] K. Ju-Nan(郭如男), C. Wei-Lun(陳維倫), and J. Wen-Yuh(覺文郁), "Surface Micromachined Adjustable Micro-Concave Mirror for Bio-Detection Applications," Chinese Physics Letters, vol. 26, 2009. 
[17] Y. S. Ow, M. B. H. Breese, and S. Azimi, "Fabrication of concave silicon micro-mirrors," Optics Express, vol. 18, pp. 14511-14518, 2010.

[18] J. L. Wilbur, R. J. Jackman, G. M. Whitesides, E. L. Cheung, L. K. Lee, and M. G. Prentiss, "Elastomeric Optics," Chem. Mater., vol. 8, pp. 1380-1385, 1996.

[19] D. V. Vezenov, B. T. Mayers, R. S. Conroy, G. M. Whitesides, P. T. Snee, Y. Chan, D. G. Nocera, and M. G. Bawendi, "A Low-Threshold, High-Efficiency Microfluidic Waveguide Laser," Journal of the Americal Chemical Society, vol. 127, pp. 8952-8953, 2005. 


\section{Chapter 2: Theoretical Basis}

\subsection{Reflection and refraction}

When light (or any kind of electromagnetic wave) propagating through a medium reaches the interface with a different medium, the phenomena known as reflection and refraction take place. Reflection refers to the fraction of light energy that is bounced back into the original medium [1, 2], while refraction refers to the energy that trespasses the interface [3] (see Figure 2.1).

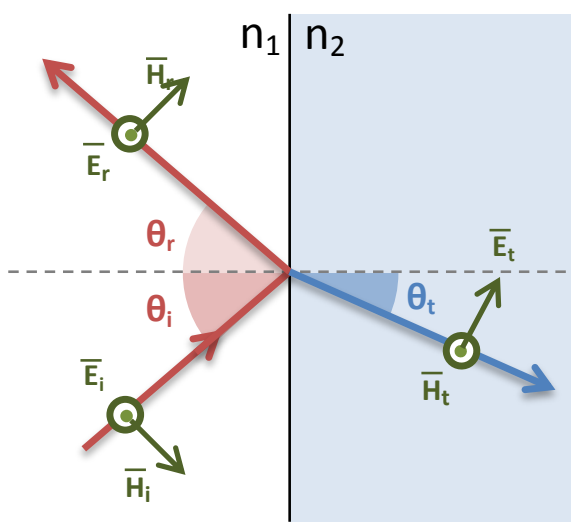

(a)

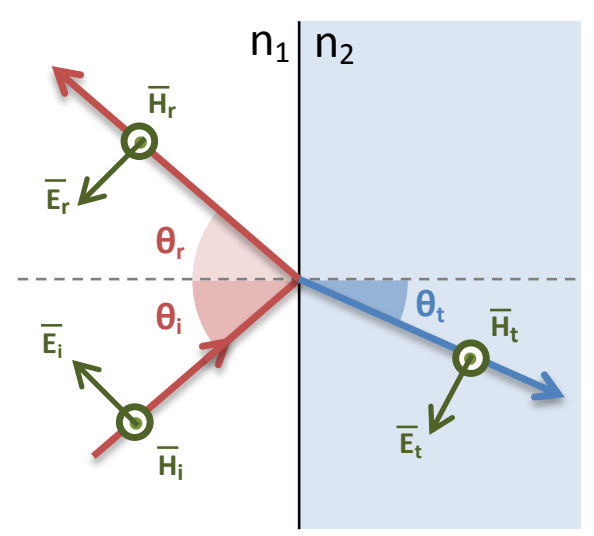

(b)

Fig. 2.1 Reflection and refraction scheme. (a) S-polarized wave; (b) p-polarized wave.

The fractions of the original electromagnetic field that bounce back and go forth are determined by two imaginary values called reflection and transmission coefficients. These values depend on the angle that light hits the interface (angle of incidence) the index of refraction of both mediums, and are calculated through Fresnel's equations (see Equation 2.1). Thus, the higher the angle of incidence, the more light reflects and less is transmitted, and the different indexes of refraction will set up the rate this happens as the angle is varied. Furthermore, we can differentiate between two types of Fresnel's coefficients regarding the polarization of light (S-polarized and P-polarized) [4].

S-polarized: The electric field vector is parallel to the plane of the interface or, in other words, perpendicular to the plane of the diagram in Figure 2.1 (see Figure 2.1.a)

P-polarized: The electric field vector belongs to a plane which is normal to the interface, such as the plane of the diagram in Figure 2.1 (see Figure 2.1.b) 


$$
\begin{array}{ll}
r_{s}=\frac{n_{1} \cos \theta_{i}-n_{2} \cos \theta_{t}}{n_{1} \cos \theta_{i}+n_{2} \cos \theta_{t}} & t_{s}=\frac{2 n_{1} \cos \theta_{i}}{n_{1} \cos \theta_{i}+n_{2} \cos \theta_{t}} \\
r_{p}=\frac{n_{1} \cos \theta_{t}-n_{2} \cos \theta_{i}}{n_{1} \cos \theta_{t}+n_{2} \cos \theta_{i}} & t_{p}=\frac{2 n_{1} \cos \theta_{i}}{n_{1} \cos \theta_{t}+n_{2} \cos \theta_{i}}
\end{array}
$$

Eq. 2.1 Fresnel equations for the reflection $(r)$ and transmission $(t)$ coefficients, in function of the polarization of the electrical field. S-polarized means that $E$ is in the plane of the interface while P-polarized means that $\mathrm{E}$ is in a plane perpendicular to the interface.

\subsection{Reflectivity and Transmissivity}

Furthermore, two more concepts can be defined, which are reflectance (or reflectivity) and transmittance (or transmissivity). They refer to the amount of light intensity (or power) that is reflected and transmitted, respectively. Reflectivity $(R)$ is calculated as the square module of the reflection coefficient, and thanks to the conservation of energy we can calculate transmittance as $T=1-R$ [4]. In case of working with a non-polarized wave, $R$ will be the arithmetic mean of $R_{s}$ and $R_{p}$ (see Equation 2.2)

$$
\begin{gathered}
R_{s}=\left|r_{s}\right|^{2}=\left|\frac{n_{1} \cos \theta_{i}-n_{2} \cos \theta_{t}}{n_{1} \cos \theta_{i}+n_{2} \cos \theta_{t}}\right|^{2}=\left|\frac{n_{1} \cos \theta_{i}-n_{2} \sqrt{1-\left(\frac{n_{1}}{n_{2}} \sin \theta_{t}\right)^{2}}}{n_{1} \cos \theta_{i}+n_{2} \sqrt{1-\left(\frac{n_{1}}{n_{2}} \sin \theta_{t}\right)^{2}}}\right|^{2} \\
R_{p}=\left|r_{p}\right|^{2}=\left|\frac{n_{1} \cos \theta_{t}-n_{2} \cos \theta_{i}}{n_{1} \cos \theta_{t}+n_{2} \cos \theta_{i}}\right|^{2}=\left|\frac{n_{1} \sqrt{1-\left(\frac{n_{1}}{n_{2}} \sin \theta_{t}\right)^{2}}-n_{2} \cos \theta_{i}}{n_{1} \sqrt{1-\left(\frac{n_{1}}{n_{2}} \sin \theta_{t}\right)^{2}}+n_{2} \cos \theta_{i}}\right|^{2} \\
R=\frac{R_{s}+R_{p}}{2}
\end{gathered}
$$

Eq. 2.2 Reflectivity calculated by Fresnel's coefficients.

As this thesis has to do with micro-mirrors and multiple internal reflection (MIR), it seems logical to focus on the concept of reflectivity (R). Thus, it will be of interest to achieve a $R$ as close as possible to $100 \%$, so the maximum light we put in the system stays in the system and 
few is lost. As has been said above, the bigger the angle of incidence into the new media is (in our case, the mirror), the more energy reflects ( $R$ increases) and the less goes through. This means that as the light hits the mirror with angles closer to 90 o the reflectivity approaches to $100 \%$. However, there is a situation in which the incident angle does not have to reach 90 o for all the light to reflect. That particular situation is known as total internal reflection (TIR).

\subsubsection{Total Internal Reflection (TIR)}

The phenomenon of TIR only takes place when light goes from a medium with a lower index of refraction $\left(n_{1}\right)$ into another one with a higher index of refraction $\left(n_{2}\right)$. When this happens, any incidence over a particular angle called critical angle will lead to a reflectivity of a $100 \%$ and no energy will be transmitted to the second medium [5]. This is the principle in which the previous devices that incorporated air mirrors were based in order to achieve high reflectivity. In fact, the value reached was a $100 \%$, since the incidence angle on the mirrors was always over the critical angle.

\subsection{Optical properties of metals}

Metals, or conducting media, having a number of free electric charges, present some differences towards light propagation compared to the rest of materials. Opposite to the dielectrics, conductivity $(\sigma)$ in metals cannot be considered zero, which causes the refractive index to be a complex number $[6,7]$.

$$
\tilde{\mathrm{n}}=n+i k
$$

Eq. 2.3 Complex index of refraction for absorbing media.

Despite this fact, Fresnel's equations remain valid, although the angles and the reflection and transmission coefficients will turn also complex, so the geometrical meaning of them will not be that obvious[7]. This complex index of refraction has also further implications which will be of interest.

The real part of the refractive index, $n$, expresses the phase speed of the wave related to its speed in vacuum, and it is the refractive index we are used to see when calculating propagation of waves through dielectric materials. The imaginary part $k$ expresses the amount of energy absorbed by the conductive medium and transformed into thermal energy. But $k$ not only limits the transmission of light along the absorbing material, but also has an effect on reflection. For $\mathrm{k}$ values that approximate to zero, we approach to a dielectric material, where $\tilde{n}=n$, while when $n$ goes to zero and $\tilde{n} \approx k$ we approach to a reflectivity $R=100 \%$ (we never reach this value since $n$ gets never completely zero). Thus, for high values of $k$ compared to $n, R$ gets large. So, in conclusion, metals, since they have a significantly big imaginary part in their index of refraction, present higher reflectivity values for all the incidence range. However, as $n$ cannot reach a value of absolute 0 , $R$ will never be equal to $100 \%$, so, in contrast, TIR will never take place (unless in the impracticable case of a 90 o angle of incidence)[6]. 


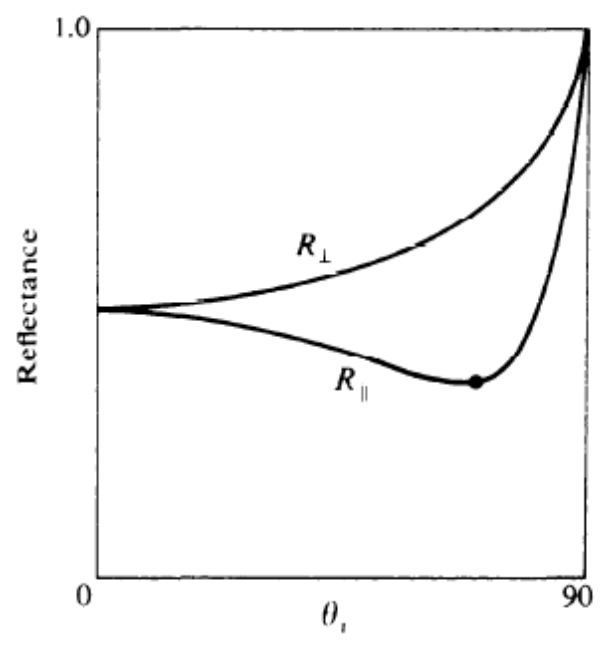

Fig. 2.2 Typical reflectance for a linearly polarized beam of white light incident on an absorbing medium.

Source: Reference [6]

\subsection{References}

[1] E. Hecht, "Reflection," in Optics, 4th ed San Francisco: Addison Wesley, 1987, pp. 95100.

[2] G. W. C. Kaye and T. H. Laby. (2005). "Light Reflection" in Tables of Physical \& Chemical Constants [Online].

Available:

http://www.kayelaby.npl.co.uk/general physics/2 $5 / 2 \quad 5 \quad 9 . h t m l$

[3] E. Hecht, "Refraction," in Optics, ed San Francisco: Addison Wesley, 1987, pp. 100-106.

[4] E. Hecht, "The Electromagnetic Approach," in Optics, ed San Francisco: Addison Wesley, 1987, pp. 111-122.

[5] E. Hecht, "Total Internal Reflection," in Optics, ed San Francisco: Addison Wesley, 1987, pp. 122-127.

[6] E. Hecht, "Optical Properties of Metals," in Optics, ed San Francisco: Addison Wesley, 1987, pp. 127-131.

[7] B. E. Sernelius. Reflection From a Metallic Surface. Electrodynamics [Online]. Available: http://www.ifm.liu.se/courses/TFYY67/Lect13.pdf 


\section{Chapter 3: Design and Theoretical Analysis of the Device}

\subsection{The microfluidic device}

\subsubsection{Description of the device}

The device that is going to be characterized in the current thesis consists on a PDMS lab-on-achip that, as has been explained in the introduction, allows performing optical detection concretely absorbance measurements-. In this section we describe the design and the different layouts of the chip, as well as we mention the techniques used for the fabrication process.

This PDMS device integrates in a monolithic design these several elements (See figure 3.1):

- Micro-fluidic channel: The micro-channel in which the sample to be analyzed is going to be poured. It is generally $1.2 \mathrm{~cm}$ long, $900 \mu \mathrm{m}$ wide and $250 \mu \mathrm{m}$ high.

- Optical fiber input and output channels: Two $250 \mu \mathrm{m}$ diameter channels intended to plug-in the input and output optical fibers.

- Optical lenses: At the end of the optical fiber channels there are two spherical lenses in order to focus the light coming out/into the fiber into parallel beams.

- Indium micro-mirrors: In the 8 different layouts of the chip, the number of mirrors intended to bounce the light back into the micro-fluidic channel in order to enlarge the optical path varies from 1 to 3 . These mirrors are made of indium to achieve a higher reflectivity and are parabolic in order to focus all the light back and avoid dispersion losses.

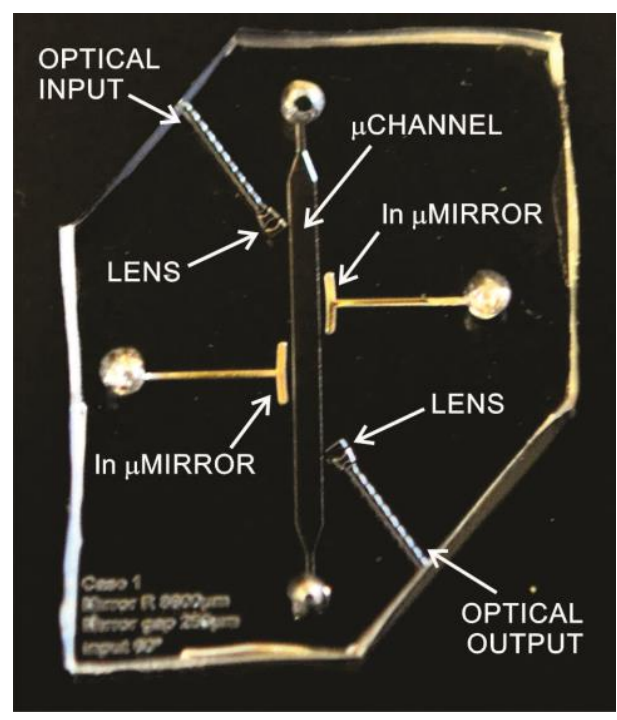

(a)

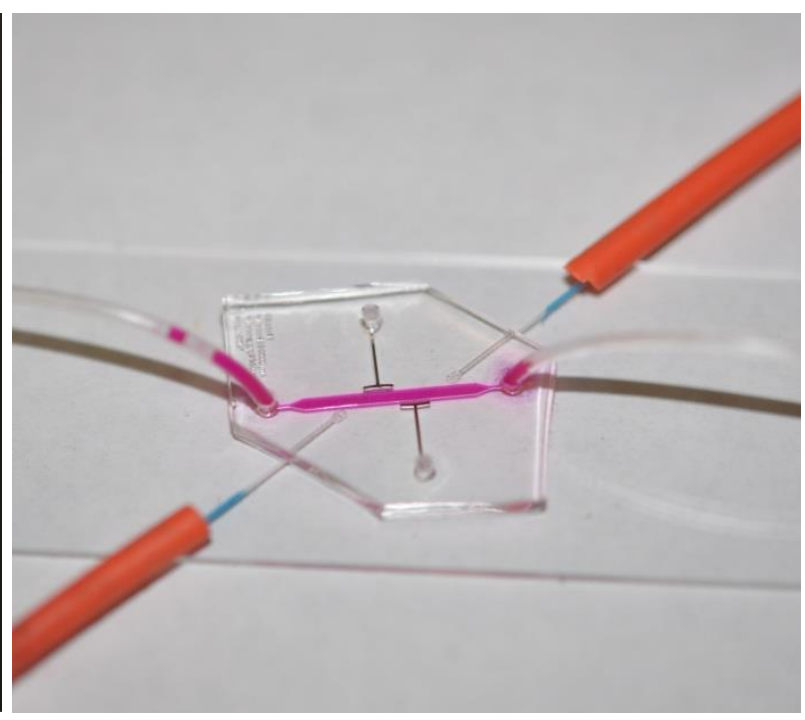

(b)

Fig. 3.1 Pictures of the device: (a) matching parts and (b) fully functional.

\subsubsection{Fabrication}

The description of the fabrication of the device is not the main aim of the current thesis. However, it is good to give the reader a basic look upon the fabrication process, which will be 
described here briefly and schematically. A more extensive and detailed description is described elsewhere[1, 2].

The device was fabricated by casting PDMS in a master made of SU-8 on a silicon wafer [3] process commonly known as soft lithography- (Figure 3.2.a). Then each chip was taken apart and sealed on a glass slide using an oxygen plasma treatment (Figure 3.2.b). Finally, the channels with the final shape of the mirrors were filled with molten indium [1] (Figure 3.2.c).

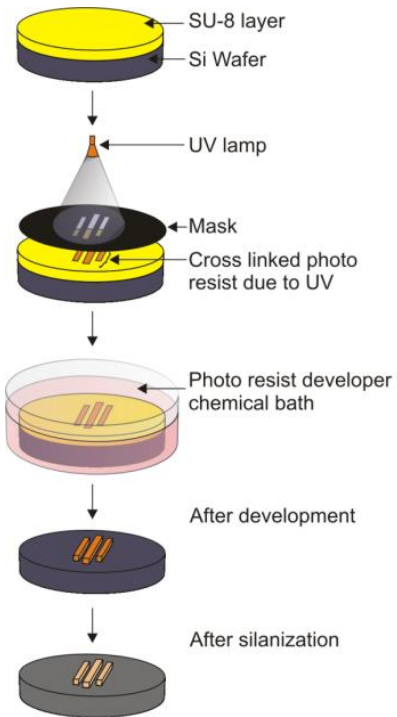

(a)

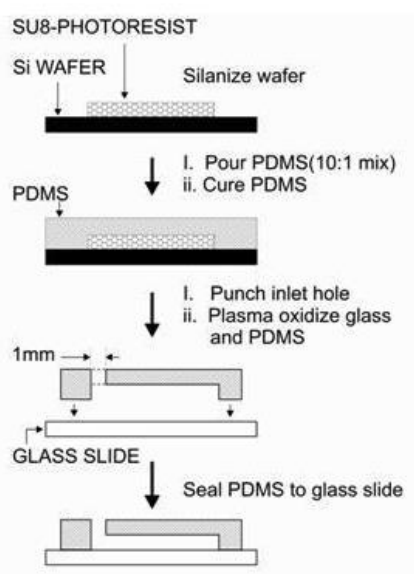

(b)

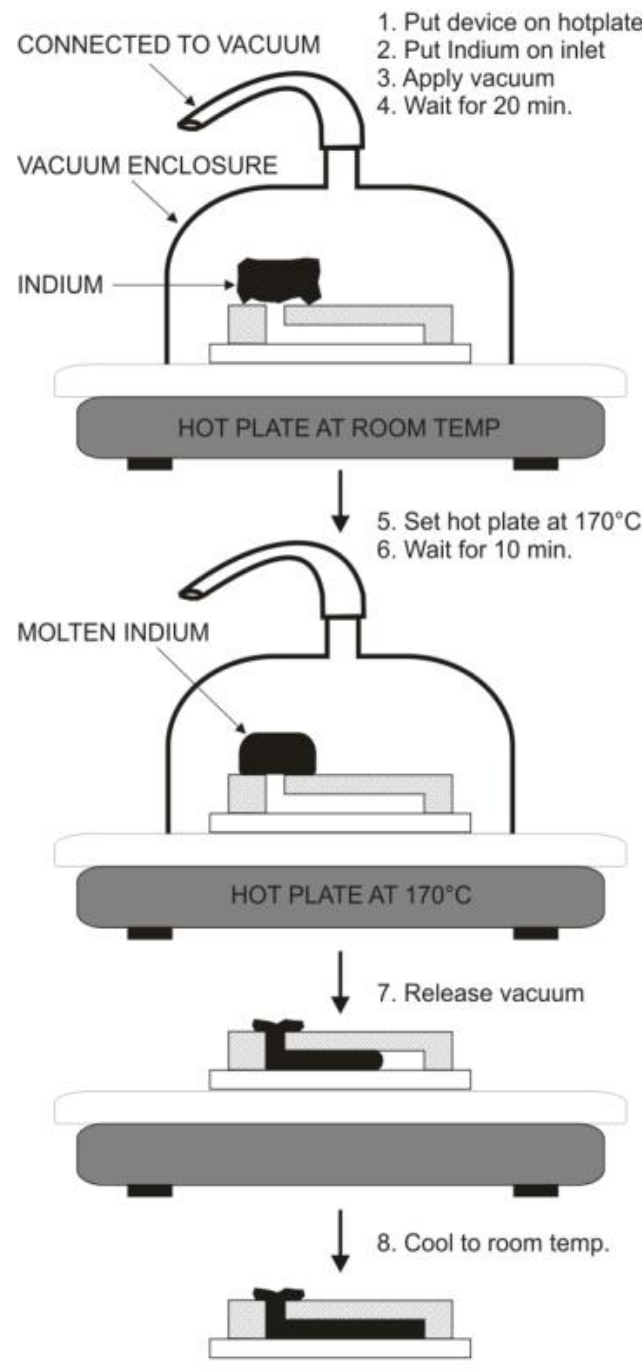

(c)

Fig. 3.2 (a) Schematic representation of the master fabrication; (b) Casting of the PDMS device and sealing of it on glass slide; (c) Process of melting the indium and filling the mirror hollows. Source: [4]

\subsubsection{Different layouts of the device}

Different configurations of the same kind of device were imprinted in the SU-8 master wafers. We varied factors such as the position and number of mirrors, the input and output angle of incidence, the micro-channel width, etc. Thus, a total number of 8 different layouts were designed and produced (the entire set is shown in Figure 3.3) 


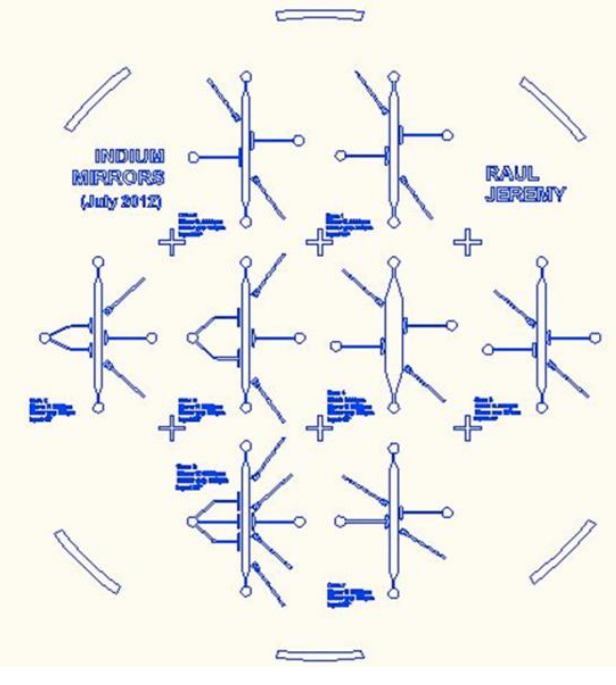

(a)

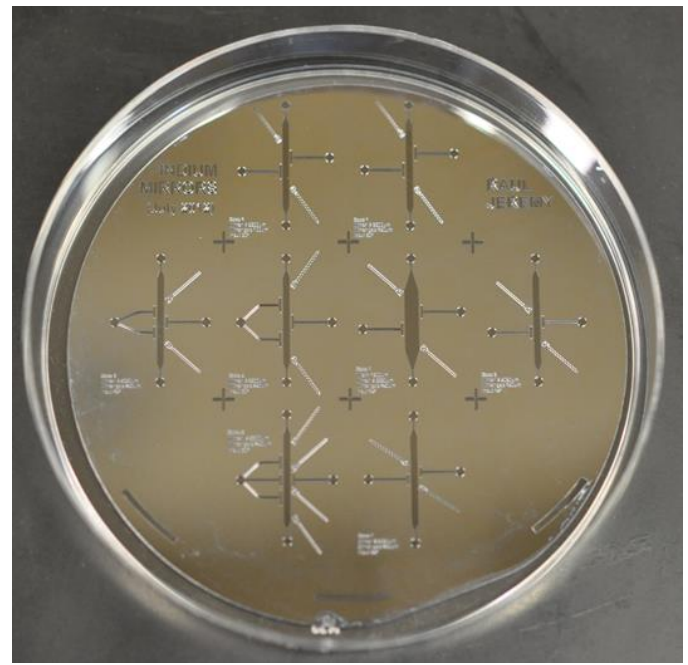

(b)

Fig. 3.3 (a) AutoCad design for photolithography mask; (b) fabricated master on a plate.

The following table contains a summary of the different parameters and its values for all the designed cases.

Table 3.1

Description of the eight layouts designed for the PDMS microfluidic device.

\begin{tabular}{llllll}
\hline Case & $\begin{array}{l}\text { Input/Output } \\
\text { Angle }\end{array}$ & Channel Width & $\begin{array}{l}\text { No. of } \\
\text { Mirrors }\end{array}$ & Optical Path Length & Necessary channel length \\
\hline $\mathbf{1}$ & $60^{\circ}$ & $900 \mu \mathrm{m}$ & 2 & $5040 \mu \mathrm{m}$ & $8000 \mu \mathrm{m}$ \\
$\mathbf{2}$ & $60^{\circ}$ & $900 \mu \mathrm{m}$ & 2 & $5165 \mu \mathrm{m}$ & $7400 \mu \mathrm{m}$ \\
$\mathbf{3}$ & $45^{\circ}$ & $900 \mu \mathrm{m}$ & 2 & $3755 \mu \mathrm{m}$ & $5200 \mu \mathrm{m}$ \\
$\mathbf{4}$ & $45^{\circ}$ & $1900 \mu \mathrm{m}$ & 2 & $5415 \mu \mathrm{m}$ & $8300 \mu \mathrm{m}$ \\
$\mathbf{5}$ & $60^{\circ}$ & $900 \mu \mathrm{m}$ & 3 & $6910 \mu \mathrm{m}$ & $10600 \mu \mathrm{m}$ \\
$\mathbf{6}$ & $45^{\circ}$ & $900 \mu \mathrm{m}$ & 3 & $5050 \mu \mathrm{m}$ & $6800 \mu \mathrm{m}$ \\
$\mathbf{7}$ & $35^{\circ}$ & $900 \mu \mathrm{m}$ & 2 & $3240 \mu \mathrm{m}$ & $3900 \mu \mathrm{m}$ \\
$\mathbf{8}$ & $60^{\circ} ; 45^{\circ}$ & $900 \mu \mathrm{m}$ & $3 ; 1$ & $6800 \mu \mathrm{m} ; 2470 \mu \mathrm{m}$ & $10600 \mu \mathrm{m} ; 3850 \mu \mathrm{m}$ \\
\hline
\end{tabular}

The first thing to notice about Table 3.1, is the similarity between cases 1 and 2, which have the same amount of mirrors and the same angle of incidence, but still, slight differences on the path length. What varies between these two layouts is the distance between the micromirrors and the channel (see Figure 3.4). All cases but number 2 are designed with a $250 \mu \mathrm{m}$ gap in order to follow have an aspect ratio of 1:1 in the soft lithography mask -i.e. a quotient between height and distance of elements big enough to assure that the PDMS does not collapse [3]-. However, case 2 was designed with a distance of only $100 \mu \mathrm{m}$ between the channel and the mirrors, expecting thus fewer losses in that transition. 


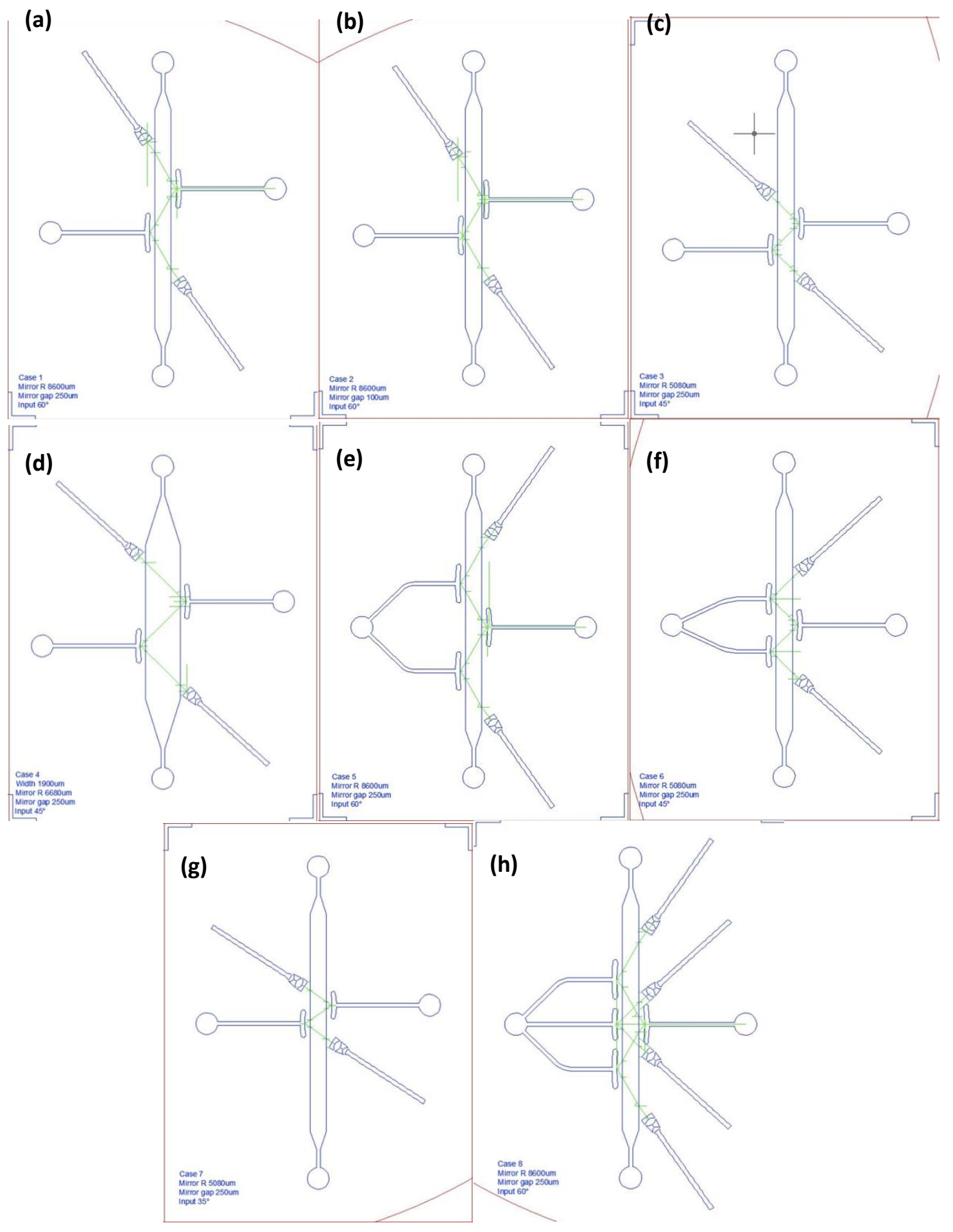

Fig. 3.4 Microfluidic device design with AutoCad for all the eight different layouts of the chip: (a) Case 1; (b) case 2; (c) case 3; (d) case 4; (e) case 5; (f) case6; (g) case 7; (h) case 8.

Source: [2]

The second thing that can be observed from Table 3.1 is that case number 8 has a pair of values for most of the parameters. The reason is that this layout incorporates two different configurations in one: three mirrors or one mirror (see Figure 3.4.h). So, case number eight is the result of merging two different layouts in one. This is a final design with the intention that the same device could be used to measure with different configurations. Thus, for lower 
concentrations where a longer optical path is needed, the three-reflection configuration can be used. However, for high concentrations likely to saturate the system with longer paths, the single-reflection configuration is more suitable.

The table also displays in the last column the necessary length of the channel. Due to the different angles of incidence of light at the input/output and to the number of mirrors integrated, the real needed length for each case is not the same, although during the design stage having a standard length for all of them was prioritized $(1.2 \mathrm{~cm})$. However, at the time of fabricating a final fully functional device, this parameter -the necessary length of the channelwill be very important in order to achieve a compact layout and to minimize the use of analyte.

These eight different layouts are meant to be tested and find out the optimal values of the limit of detection, sensitivity and compactness that can be achieved, and their dependence with the path-length, the amount of mirrors and the incidence angles.

\subsection{Mirrors made of indium}

\subsubsection{Advantages of a metallic mirror}

The device under study in this thesis, as has been said so far, incorporates indium mirrors to enlarge the optical path of light by generating multiple internal reflections. Some other research groups have developed this kind of device using air instead of metallic mirrors $[5,6]$ (see Figure 3.5).

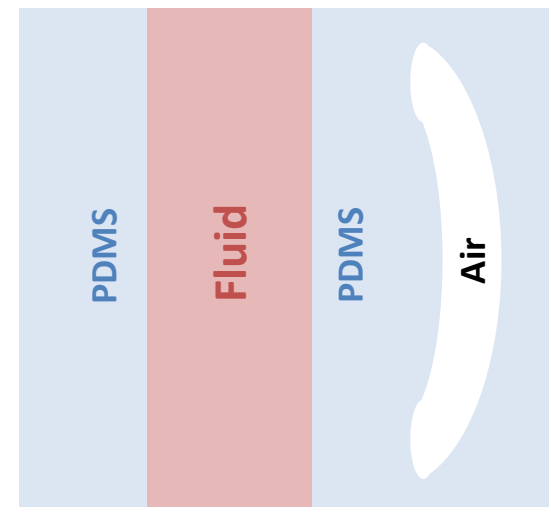

(a)

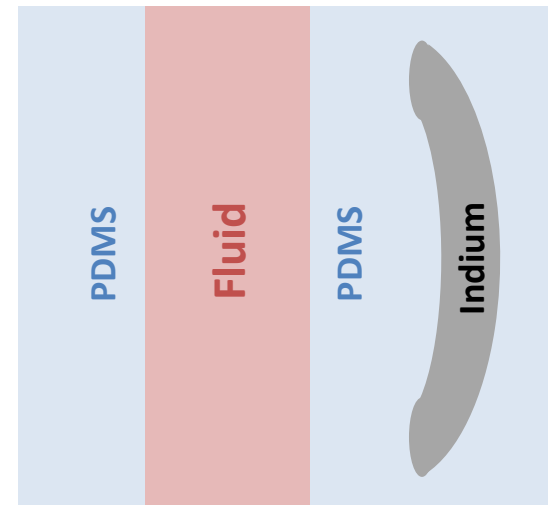

(b)

Fig. 3.5 Scheme of the micro mirrors inside the chip. (a) Air micro mirror of the elder model; (b) Indium mirror of the new model.

As explained in Chapter 2 (Section 2.3), a metallic mirror has both advantages and disadvantages compared to a non-conductive mirror like air.

Disadvantage: Metals, being an absorbing medium, do not have a region of incidence in which TIR can be achieved, so a reflectivity (R) of completely $100 \%$ will never be possible.

Advantage: Metallic mirrors, having a complex index of refraction ( $\tilde{n}=n+i k)$ with $k$ high enough compared to $\mathrm{n}$ (typically between 5 and 10 times bigger in the visible spectrum), present large values for $\mathrm{R}$ (typically around $85 \%$ for indium) no matter the angle of incidence (see Table 3.2 ). 
This is interesting since, reducing the angle of incidence leads to a more compact integration of the whole system and thus, among other advantages, less amount of fluid is needed to perform the analysis (see Figure 3.6).

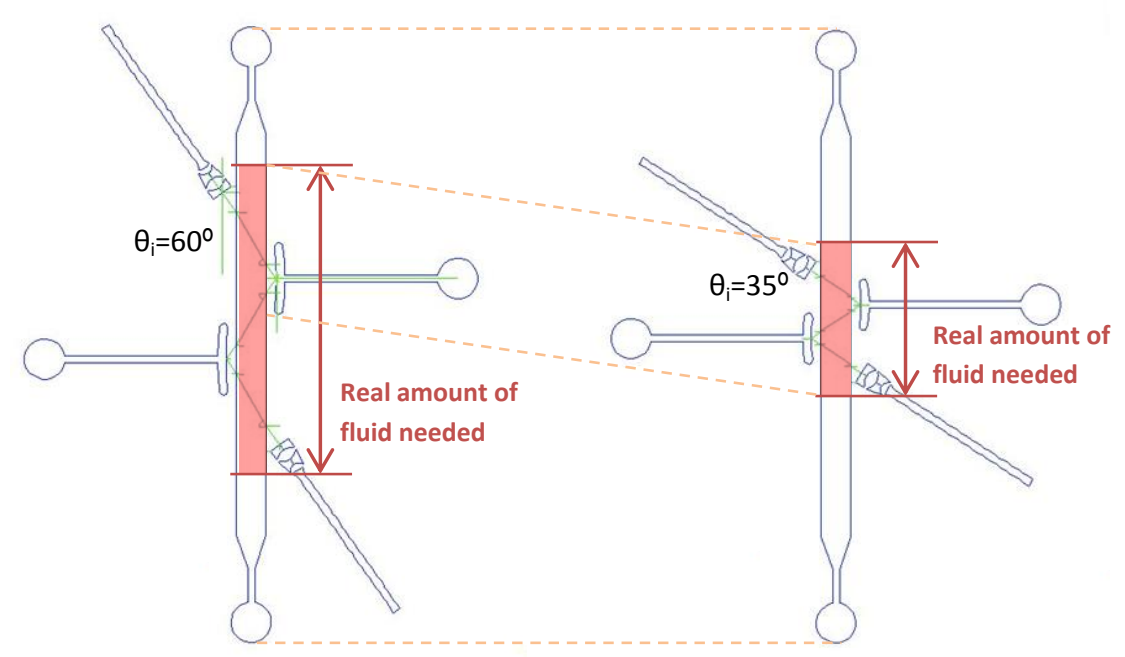

Fig. 3.6 Scheme of the chip matching the reduction of the amount of fluid needed (approximately half) if $\theta_{i}$ is reduced from $60^{\circ}$ to $35^{\circ}$.

This situation leads then to a question: ¿Do the advantages overcome the disadvantages of using metallic mirrors? The truth is that there are some applications that require mirrors that work with low or even normal angles of incidence [7] and that can only be achieved with metals. Furthermore, the goal of this thesis is not actually improving a previous design of an absorbance device [5] but researching on the feasibility of integrating metallic mirrors for microfluidic applications and the quality that can be achieved in them.

\subsubsection{Reason for choosing Indium}

There are several benefits to the selection of indium for fabricating micromirrors integrated to microfluidic devices:

- The reflectance of indium remains approximately constant for the wavelengths of interest-i.e., the visible spectrum $(400-800 \mathrm{~nm}$, Figure 3.7)-differently from common metals such as gold, silver, or aluminum.

- The cost of indium $(\sim 500 / \mathrm{kg})$ is similar to that of silver and much lower than that of aluminum $(\sim \$ 2300 / \mathrm{kg})$ or gold $(\sim \$ 30,500 / \mathrm{kg})[8]$.

- The melting point of indium $\left(\sim 156^{\circ} \mathrm{C}\right)$ is much lower than most metals: $1064^{\circ} \mathrm{C}, 962^{\circ} \mathrm{C}$, and $660^{\circ} \mathrm{C}$ for gold, silver, and aluminum, respectively. This characteristic makes it possible to form microstructures of indium simply by filling PDMS channels with molten indium [1]. 


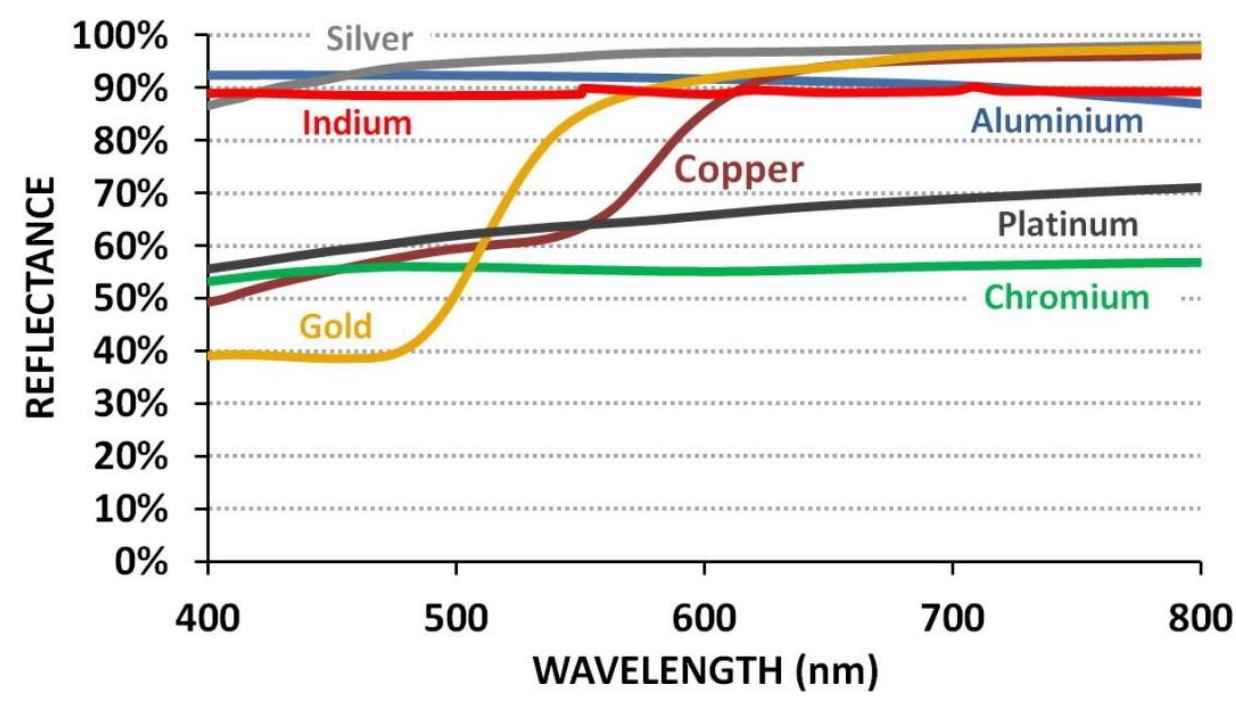

Fig. 3.7 Dependence of the reflectance $(R)$ of various metals upon the inciding wavelength. For this figure we assume that the light beam travels through air (nair=1) before inciding the metal surface perpendicularly. Curves in this figure were calculated using Fresnel equations for reflectivity explained in Chapter 2 (Equation 2.2) taking into account that $\mathrm{n}$ for metals is a complex number and it has to be plugged into the equations with its real and imaginary part.

\subsubsection{Theoretical reflectivity of the indium micromirrors}

Optical data for the whole fluid-PDMS-indium system (assuming $\mathrm{H}_{2} \mathrm{O}$ as the fluid since it is the solvent employed in the solutions under test) are going to be presented below, as well as the theoretical calculations for reflectivity $(R)$, transmittance $(T)$, and the different possible angles of the light beams (incident, reflected and refracted). These calculations only contemplate a single reflection on a mirror from the micro-fluidic channel, although they mean several media changes. Figure 3.8 shows a diagram with the different reflected and refracted beams and the name by which all the angles are going to be referenced.

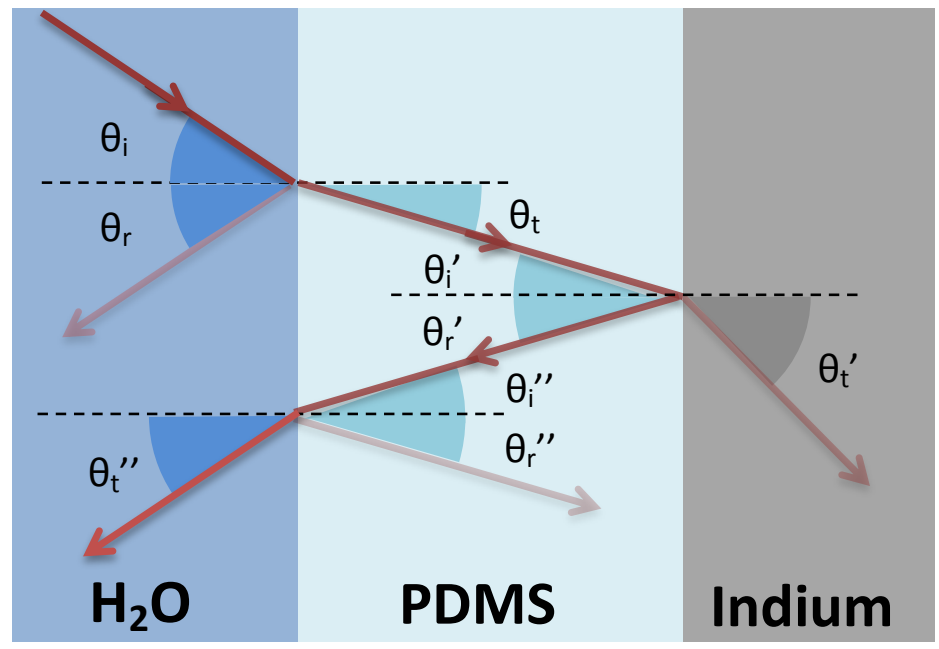

Fig. 3.8 Diagram of the water-PDMS-indium system that contemplates all the media changes that take place in a single reflection in the micro-channel. 
Table 3.2 shows the different values of the angles represented in Figure 3.8, as well as the transmittance from water to PDMS ( $T_{\text {H2O-PDMS }}$ ), the reflectance from indium to PDMS (Rs PDMS-In $_{\text {; }}$ $\mathrm{Rp}_{\text {PDMS -In }}$ ) and the transmittance back to water from PDMS ( $\left.T_{\text {PDMS-Hzo }}\right)$. The total percentage of light intensity that is not lost in this whole process is represented in the last column ( $\left.R_{\text {overall }}\right)$. All those values depend on the wavelength of light $(\lambda)$ and the first incidence angle $\left(\theta_{i}\right)$, which correspond to the first two columns.

All reflectivity and transmittance (1-R) values in Table 3.2 have been calculated using Fresnel's reflection coefficients in the form written in Equation 2.2. (Chapter 2, Section 2.2)

Table 3.2

Optical values on the reflection process from water on a two-layers system of PDMS and Indium. All the values are in function of the two parameters in the two first columns. The last column contains the value of the total reflectivity on de multi-layer system.

\begin{tabular}{|c|c|c|c|c|c|c|c|c|c|c|c|c|}
\hline $\begin{array}{l}\bar{\xi} \\
\underline{\underline{\varepsilon}}\end{array}$ & $0^{5}$ & 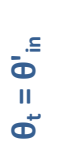 & $\begin{array}{c}\subseteq \\
\overline{0} \\
11 \\
\sigma^{2}\end{array}$ & $\bar{\theta}^{+}$ & ঙ & $\sum_{\substack{0 \\
\varrho}}^{n}$ & $\underset{\text { a }}{\text { a }}$ & 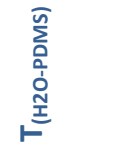 & 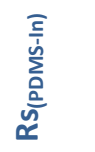 & 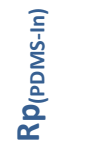 & 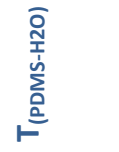 & 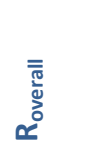 \\
\hline 310 & $0^{\circ}$ & $0.00^{\circ}$ & $0.00^{\circ}$ & $0^{\circ}$ & 1.3478 & 1.4 & $0.38+3 i$ & $99.96 \%$ & $82.5 \%$ & $82.5 \%$ & $99.96 \%$ & $82.45 \%$ \\
\hline 354 & $0^{\circ}$ & $0.00^{\circ}$ & $0.00^{\circ}$ & $0^{\circ}$ & 1.3427 & 1.4 & $0.4+3.4 i$ & $99.96 \%$ & $84.9 \%$ & $84.9 \%$ & $99.96 \%$ & $84.79 \%$ \\
\hline 381 & $0^{\circ}$ & $0.00^{\circ}$ & $0.00^{\circ}$ & $0^{\circ}$ & 1.3405 & 1.4 & $0.45+3.7 i$ & $99.95 \%$ & $85.3 \%$ & $85.3 \%$ & $99.95 \%$ & $85.19 \%$ \\
\hline 413 & $0^{\circ}$ & $0.00^{\circ}$ & $0.00^{\circ}$ & $0^{\circ}$ & 1.3385 & 1.4 & $0.5+4 i$ & $99.95 \%$ & $85.7 \%$ & $85.7 \%$ & $99.95 \%$ & $85.64 \%$ \\
\hline 451 & $0^{\circ}$ & $0.00^{\circ}$ & $0.00^{\circ}$ & $0^{\circ}$ & 1.3370 & 1.4 & $0.6+4.3 i$ & $99.95 \%$ & $85.1 \%$ & $85.1 \%$ & $99.95 \%$ & $84.97 \%$ \\
\hline 496 & $0^{\circ}$ & $0.00^{\circ}$ & $0.00^{\circ}$ & $0^{\circ}$ & 1.3352 & 1.4 & $0.75+4.8 \mathrm{i}$ & $99.94 \%$ & $84.8 \%$ & $84.8 \%$ & $99.94 \%$ & $84.72 \%$ \\
\hline 550 & $0^{\circ}$ & $0.00^{\circ}$ & $0.00^{\circ}$ & $0^{\circ}$ & 1.3330 & 1.4 & $0.7+4.7 i$ & $99.94 \%$ & $85.2 \%$ & $85.2 \%$ & $99.94 \%$ & $85.11 \%$ \\
\hline 551 & $0^{\circ}$ & $0.00^{\circ}$ & $0.00^{\circ}$ & $0^{\circ}$ & 1.3330 & 1.4 & $0.85+5.5 i$ & $99.94 \%$ & $86.5 \%$ & $86.5 \%$ & $99.94 \%$ & $86.42 \%$ \\
\hline 600 & $0^{\circ}$ & $0.00^{\circ}$ & $0.00^{\circ}$ & $0^{\circ}$ & 1.3320 & 1.4 & $0.8+5 i$ & $99.94 \%$ & $85.0 \%$ & $85.0 \%$ & $99.94 \%$ & $84.88 \%$ \\
\hline 620 & $0^{\circ}$ & $0.00^{\circ}$ & $0.00^{\circ}$ & $0^{\circ}$ & 1.3320 & 1.4 & $1.05+6 i$ & $99.94 \%$ & $86.0 \%$ & $86.0 \%$ & $99.94 \%$ & $85.89 \%$ \\
\hline 650 & $0^{\circ}$ & $0.00^{\circ}$ & $0.00^{\circ}$ & $0^{\circ}$ & 1.3310 & 1.4 & $0.9+5.42 i$ & $99.94 \%$ & $85.5 \%$ & $85.5 \%$ & $99.94 \%$ & $85.35 \%$ \\
\hline
\end{tabular}

\begin{tabular}{|c|c|c|c|c|c|c|c|c|c|c|c|c|}
\hline 310 & $10^{\circ}$ & $9.62^{\circ}$ & $9.62^{\circ}$ & $10^{\circ}$ & 1.3478 & 1.4 & $0.38+3 i$ & $99.96 \%$ & $82.8 \%$ & $82.2 \%$ & $99.96 \%$ & $82.45 \%$ \\
\hline 354 & $10^{\circ}$ & $9.59^{\circ}$ & $9.59^{\circ}$ & $10^{\circ}$ & 1.3427 & 1.4 & $0.4+3.4 \mathrm{i}$ & $99.96 \%$ & $85.1 \%$ & $84.6 \%$ & $99.96 \%$ & $84.79 \%$ \\
\hline 381 & $10^{\circ}$ & $9.57^{\circ}$ & $9.57^{\circ}$ & $10^{\circ}$ & 1.3405 & 1.4 & $0.45+3.7 i$ & $99.95 \%$ & $85.5 \%$ & $85.1 \%$ & $99.95 \%$ & $85.19 \%$ \\
\hline 413 & $10^{\circ}$ & $9.56^{\circ}$ & $9.56^{\circ}$ & $10^{\circ}$ & 1.3385 & 1.4 & $0.5+4 i$ & $99.95 \%$ & $85.9 \%$ & $85.5 \%$ & $99.95 \%$ & $85.63 \%$ \\
\hline 451 & $10^{\circ}$ & $9.55^{\circ}$ & $9.55^{\circ}$ & $10^{\circ}$ & 1.3370 & 1.4 & $0.6+4.3 i$ & $99.95 \%$ & $85.3 \%$ & $84.8 \%$ & $99.95 \%$ & $84.97 \%$ \\
\hline 496 & $10^{\circ}$ & $9.53^{\circ}$ & $9.53^{\circ}$ & $10^{\circ}$ & 1.3352 & 1.4 & $0.75+4.8 i$ & $99.94 \%$ & $85.0 \%$ & $84.6 \%$ & $99.94 \%$ & $84.72 \%$ \\
\hline 550 & $10^{\circ}$ & $9.52^{\circ}$ & $9.52^{\circ}$ & $10^{\circ}$ & 1.3330 & 1.4 & $0.7+4.7 i$ & $99.94 \%$ & $85.4 \%$ & $85.0 \%$ & $99.94 \%$ & $85.10 \%$ \\
\hline 551 & $10^{\circ}$ & $9.52^{\circ}$ & $9.52^{\circ}$ & $10^{\circ}$ & 1.3330 & 1.4 & $0.85+5.5 i$ & $99.94 \%$ & $86.7 \%$ & $86.3 \%$ & $99.94 \%$ & $86.42 \%$ \\
\hline 600 & $10^{\circ}$ & $9.51^{\circ}$ & $9.51^{\circ}$ & $10^{\circ}$ & 1.3320 & 1.4 & $0.8+5 i$ & $99.94 \%$ & $85.2 \%$ & $84.8 \%$ & $99.94 \%$ & $84.88 \%$ \\
\hline 620 & $10^{\circ}$ & $9.51^{\circ}$ & $9.51^{\circ}$ & $10^{\circ}$ & 1.3320 & 1.4 & $1.05+6 i$ & $99.94 \%$ & $86.2 \%$ & $85.8 \%$ & $99.94 \%$ & $85.89 \%$ \\
\hline 650 & $10^{\circ}$ & $9.50^{\circ}$ & $9.50^{\circ}$ & $10^{\circ}$ & 1.3310 & 1.4 & $0.9+5.42 i$ & $99.94 \%$ & $85.7 \%$ & $85.3 \%$ & $99.94 \%$ & $85.35 \%$ \\
\hline 310 & $20^{\circ}$ & $19.22^{\circ}$ & $19.22^{\circ}$ & $20^{\circ}$ & 1.3478 & 1.4 & $0.38+3 i$ & $99.96 \%$ & $83.6 \%$ & $81.4 \%$ & $99.96 \%$ & $82.45 \%$ \\
\hline 354 & $20^{\circ}$ & $19.15^{\circ}$ & $19.15^{\circ}$ & $20^{\circ}$ & 1.3427 & 1.4 & $0.4+3.4 i$ & $99.96 \%$ & $85.8 \%$ & $84.0 \%$ & $99.96 \%$ & $84.78 \%$ \\
\hline 381 & $20^{\circ}$ & $19.12^{\circ}$ & $19.12^{\circ}$ & $20^{\circ}$ & 1.3405 & 1.4 & $0.45+3.7 i$ & $99.95 \%$ & $86.1 \%$ & $84.4 \%$ & $99.95 \%$ & $85.18 \%$ \\
\hline
\end{tabular}




\begin{tabular}{|c|c|c|c|c|c|c|c|c|c|c|c|c|}
\hline 413 & $20^{\circ}$ & $19.09^{\circ}$ & $19.09^{\circ}$ & $20^{\circ}$ & 1.3385 & 1.4 & $0.5+4 i$ & $99.95 \%$ & $86.5 \%$ & $84.9 \%$ & $99.95 \%$ & $85.62 \%$ \\
\hline 451 & $20^{\circ}$ & $19.06^{\circ}$ & $19.06^{\circ}$ & $20^{\circ}$ & 1.3370 & 1.4 & $0.6+4.3 i$ & $99.95 \%$ & $85.9 \%$ & $84.2 \%$ & $99.95 \%$ & $84.96 \%$ \\
\hline 496 & $20^{\circ}$ & $19.04^{\circ}$ & $19.04^{\circ}$ & $20^{\circ}$ & 1.3352 & 1.4 & $0.75+4.8 \mathrm{i}$ & $99.94 \%$ & $85.6 \%$ & $84.0 \%$ & $99.94 \%$ & $84.71 \%$ \\
\hline 550 & $20^{\circ}$ & $19.01^{\circ}$ & $19.01^{\circ}$ & $20^{\circ}$ & 1.3330 & 1.4 & $0.7+4.7 i$ & $99.94 \%$ & $86.0 \%$ & $84.4 \%$ & $99.94 \%$ & $85.09 \%$ \\
\hline 551 & $20^{\circ}$ & $19.01^{\circ}$ & $19.01^{\circ}$ & $20^{\circ}$ & 1.3330 & 1.4 & $0.85+5.5 i$ & $99.94 \%$ & $87.2 \%$ & $85.8 \%$ & $99.94 \%$ & $86.40 \%$ \\
\hline 600 & $20^{\circ}$ & $18.99^{\circ}$ & $18.99^{\circ}$ & $20^{\circ}$ & 1.3320 & 1.4 & $0.8+5 i$ & $99.94 \%$ & $85.8 \%$ & $84.2 \%$ & $99.94 \%$ & $84.87 \%$ \\
\hline 620 & $20^{\circ}$ & $18.99^{\circ}$ & $18.99^{\circ}$ & $20^{\circ}$ & 1.3320 & 1.4 & $1.05+6 i$ & $99.94 \%$ & $86.7 \%$ & $85.2 \%$ & $99.94 \%$ & $85.88 \%$ \\
\hline 650 & $20^{\circ}$ & $18.98^{\circ}$ & $18.98^{\circ}$ & $20^{\circ}$ & 1.3310 & 1.4 & $0.9+5.42 i$ & $99.94 \%$ & $86.2 \%$ & $84.7 \%$ & $99.94 \%$ & $85.34 \%$ \\
\hline 310 & $30^{\circ}$ & $28.77^{\circ}$ & $28.77^{\circ}$ & $30^{\circ}$ & 1.3478 & 1.4 & $0.38+3 i$ & $99.96 \%$ & $84.8 \%$ & $80.2 \%$ & $99.96 \%$ & $82.43 \%$ \\
\hline 354 & $30^{\circ}$ & $28.65^{\circ}$ & $28.65^{\circ}$ & $30^{\circ}$ & 1.3427 & 1.4 & $0.4+3.4 i$ & $99.95 \%$ & $86.8 \%$ & $82.8 \%$ & $99.95 \%$ & $84.75 \%$ \\
\hline 381 & $30^{\circ}$ & $28.60^{\circ}$ & $28.60^{\circ}$ & $30^{\circ}$ & 1.3405 & 1.4 & $0.45+3.7 i$ & $99.95 \%$ & $87.1 \%$ & $83.3 \%$ & $99.95 \%$ & $85.14 \%$ \\
\hline 413 & $30^{\circ}$ & $28.56^{\circ}$ & $28.56^{\circ}$ & $30^{\circ}$ & 1.3385 & 1.4 & $0.5+4 i$ & $99.94 \%$ & $87.5 \%$ & $83.8 \%$ & $99.94 \%$ & $85.57 \%$ \\
\hline 451 & $30^{\circ}$ & $28.52^{\circ}$ & $28.52^{\circ}$ & $30^{\circ}$ & 1.3370 & 1.4 & $0.6+4.3 i$ & $99.94 \%$ & $86.9 \%$ & $83.1 \%$ & $99.94 \%$ & $84.90 \%$ \\
\hline 496 & $30^{\circ}$ & $28.48^{\circ}$ & $28.48^{\circ}$ & $30^{\circ}$ & 1.3352 & 1.4 & $0.75+4.8 \mathrm{i}$ & $99.94 \%$ & $86.6 \%$ & $82.9 \%$ & $99.94 \%$ & $84.64 \%$ \\
\hline 550 & $30^{\circ}$ & $28.43^{\circ}$ & $28.43^{\circ}$ & $30^{\circ}$ & 1.3330 & 1.4 & $0.7+4.7 i$ & $99.93 \%$ & $87.0 \%$ & $83.3 \%$ & $99.93 \%$ & $85.03 \%$ \\
\hline 551 & $30^{\circ}$ & $28.43^{\circ}$ & $28.43^{\circ}$ & $30^{\circ}$ & 1.3330 & 1.4 & $0.85+5.5 \mathrm{i}$ & $99.93 \%$ & $88.1 \%$ & $84.8 \%$ & $99.93 \%$ & $86.34 \%$ \\
\hline 600 & $30^{\circ}$ & $28.41^{\circ}$ & $28.41^{\circ}$ & $30^{\circ}$ & 1.3320 & 1.4 & $0.8+5 i$ & $99.93 \%$ & $86.8 \%$ & $83.1 \%$ & $99.93 \%$ & $84.80 \%$ \\
\hline 620 & $30^{\circ}$ & $28.41^{\circ}$ & $28.41^{\circ}$ & $30^{\circ}$ & 1.3320 & 1.4 & $1.05+6 i$ & $99.93 \%$ & $87.6 \%$ & $84.2 \%$ & $99.93 \%$ & $85.81 \%$ \\
\hline 650 & $30^{\circ}$ & $28.38^{\circ}$ & $28.38^{\circ}$ & $30^{\circ}$ & 1.3310 & 1.4 & $0.9+5.42 i$ & $99.93 \%$ & $87.2 \%$ & $83.6 \%$ & $99.93 \%$ & $85.27 \%$ \\
\hline 310 & $40^{\circ}$ & $38.23^{\circ}$ & $38.23^{\circ}$ & $40^{\circ}$ & 1.3478 & 1.4 & $0.38+3 i$ & $99.95 \%$ & $86.5 \%$ & $78.5 \%$ & $99.95 \%$ & $82.42 \%$ \\
\hline 354 & $40^{\circ}$ & $38.06^{\circ}$ & $38.06^{\circ}$ & $40^{\circ}$ & 1.3427 & 1.4 & $0.4+3.4 i$ & $99.94 \%$ & $88.2 \%$ & $81.3 \%$ & $99.94 \%$ & $84.67 \%$ \\
\hline 381 & $40^{\circ}$ & $37.99^{\circ}$ & $37.99^{\circ}$ & $40^{\circ}$ & 1.3405 & 1.4 & $0.45+3.7 i$ & $99.93 \%$ & $88.5 \%$ & $81.8 \%$ & $99.93 \%$ & $85.03 \%$ \\
\hline 413 & $40^{\circ}$ & $37.92^{\circ}$ & $37.92^{\circ}$ & $40^{\circ}$ & 1.3385 & 1.4 & $0.5+4 i$ & $99.93 \%$ & $88.8 \%$ & $82.3 \%$ & $99.93 \%$ & $85.45 \%$ \\
\hline 451 & $40^{\circ}$ & $37.87^{\circ}$ & $37.87^{\circ}$ & $40^{\circ}$ & 1.3370 & 1.4 & $0.6+4.3 i$ & $99.92 \%$ & $88.2 \%$ & $81.5 \%$ & $99.92 \%$ & $84.75 \%$ \\
\hline 496 & $40^{\circ}$ & $37.81^{\circ}$ & $37.81^{\circ}$ & $40^{\circ}$ & 1.3352 & 1.4 & $0.75+4.8 \mathrm{i}$ & $99.92 \%$ & $88.0 \%$ & $81.2 \%$ & $99.92 \%$ & $84.47 \%$ \\
\hline 550 & $40^{\circ}$ & $37.74^{\circ}$ & $37.74^{\circ}$ & $40^{\circ}$ & 1.3330 & 1.4 & $0.7+4.7 i$ & $99.91 \%$ & $88.3 \%$ & $81.7 \%$ & $99.91 \%$ & $84.86 \%$ \\
\hline 551 & $40^{\circ}$ & $37.74^{\circ}$ & $37.74^{\circ}$ & $40^{\circ}$ & 1.3330 & 1.4 & $0.85+5.5 i$ & $99.91 \%$ & $89.3 \%$ & $83.3 \%$ & $99.91 \%$ & $86.15 \%$ \\
\hline 600 & $40^{\circ}$ & $37.70^{\circ}$ & $37.70^{\circ}$ & $40^{\circ}$ & 1.3320 & 1.4 & $0.8+5 i$ & $99.91 \%$ & $88.1 \%$ & $81.5 \%$ & $99.91 \%$ & $84.62 \%$ \\
\hline 620 & $40^{\circ}$ & $37.70^{\circ}$ & $37.70^{\circ}$ & $40^{\circ}$ & 1.3320 & 1.4 & $1.05+6 i$ & $99.91 \%$ & $88.9 \%$ & $82.7 \%$ & $99.91 \%$ & $85.61 \%$ \\
\hline 650 & $40^{\circ}$ & $37.67^{\circ}$ & $37.67^{\circ}$ & $40^{\circ}$ & 1.3310 & 1.4 & $0.9+5.42 i$ & $99.91 \%$ & $88.4 \%$ & $82.0 \%$ & $99.91 \%$ & $85.08 \%$ \\
\hline 310 & $50^{\circ}$ & $47.52^{\circ}$ & $47.52^{\circ}$ & $50^{\circ}$ & 1.3478 & 1.4 & $0.38+3 i$ & $99.90 \%$ & $88.4 \%$ & $76.7 \%$ & $99.90 \%$ & $82.42 \%$ \\
\hline 354 & $50^{\circ}$ & $47.28^{\circ}$ & $47.28^{\circ}$ & $50^{\circ}$ & 1.3427 & 1.4 & $0.4+3.4 \mathrm{i}$ & $99.88 \%$ & $89.9 \%$ & $79.5 \%$ & $99.88 \%$ & $84.52 \%$ \\
\hline 381 & $50^{\circ}$ & $47.18^{\circ}$ & $47.18^{\circ}$ & $50^{\circ}$ & 1.3405 & 1.4 & $0.45+3.7 i$ & $99.88 \%$ & $90.1 \%$ & $80.0 \%$ & $99.88 \%$ & $84.81 \%$ \\
\hline 413 & $50^{\circ}$ & $47.09^{\circ}$ & $47.09^{\circ}$ & $50^{\circ}$ & 1.3385 & 1.4 & $0.5+4 i$ & $99.87 \%$ & $90.3 \%$ & $80.5 \%$ & $99.87 \%$ & $85.16 \%$ \\
\hline 451 & $50^{\circ}$ & $47.02^{\circ}$ & $47.02^{\circ}$ & $50^{\circ}$ & 1.3370 & 1.4 & $0.6+4.3 i$ & $99.86 \%$ & $89.8 \%$ & $79.5 \%$ & $99.86 \%$ & $84.42 \%$ \\
\hline 496 & $50^{\circ}$ & $46.93^{\circ}$ & $46.93^{\circ}$ & $50^{\circ}$ & 1.3352 & 1.4 & $0.75+4.8 \mathrm{i}$ & $99.85 \%$ & $89.6 \%$ & $79.1 \%$ & $99.85 \%$ & $84.08 \%$ \\
\hline 550 & $50^{\circ}$ & $46.83^{\circ}$ & $46.83^{\circ}$ & $50^{\circ}$ & 1.3330 & 1.4 & $0.7+4.7 i$ & $99.84 \%$ & $89.8 \%$ & $79.6 \%$ & $99.84 \%$ & $84.48 \%$ \\
\hline 551 & $50^{\circ}$ & $46.83^{\circ}$ & $46.83^{\circ}$ & $50^{\circ}$ & 1.3330 & 1.4 & $0.85+5.5 i$ & $99.84 \%$ & $90.7 \%$ & $81.3 \%$ & $99.84 \%$ & $85.72 \%$ \\
\hline 600 & $50^{\circ}$ & $46.79^{\circ}$ & $46.79^{\circ}$ & $50^{\circ}$ & 1.3320 & 1.4 & $0.8+5 i$ & $99.84 \%$ & $89.7 \%$ & $79.3 \%$ & $99.84 \%$ & $84.20 \%$ \\
\hline
\end{tabular}




\begin{tabular}{|c|c|c|c|c|c|c|c|c|c|c|c|c|}
\hline 620 & $50^{\circ}$ & $46.79^{\circ}$ & $46.79^{\circ}$ & $50^{\circ}$ & 1.3320 & 1.4 & $1.05+6 i$ & $99.84 \%$ & $90.3 \%$ & $80.5 \%$ & $99.84 \%$ & $85.14 \%$ \\
\hline 650 & $50^{\circ}$ & $46.74^{\circ}$ & $46.74^{\circ}$ & $50^{\circ}$ & 1.3310 & 1.4 & $0.9+5.42 i$ & $99.83 \%$ & $90.0 \%$ & $79.9 \%$ & $99.83 \%$ & $84.63 \%$ \\
\hline 310 & $60^{\circ}$ & $56.48^{\circ}$ & $56.48^{\circ}$ & $60^{\circ}$ & 1.3478 & 1.4 & $0.38+3 i$ & $99.72 \%$ & $90.6 \%$ & $75.3 \%$ & $99.72 \%$ & $82.46 \%$ \\
\hline 354 & $60^{\circ}$ & $56.16^{\circ}$ & $56.16^{\circ}$ & $60^{\circ}$ & 1.3427 & 1.4 & $0.4+3.4 \mathrm{i}$ & $99.67 \%$ & $91.7 \%$ & $77.8 \%$ & $99.67 \%$ & $84.20 \%$ \\
\hline 381 & $60^{\circ}$ & $56.02^{\circ}$ & $56.02^{\circ}$ & $60^{\circ}$ & 1.3405 & 1.4 & $0.45+3.7 i$ & $99.64 \%$ & $91.8 \%$ & $78.0 \%$ & $99.64 \%$ & $84.32 \%$ \\
\hline 413 & $60^{\circ}$ & $55.89^{\circ}$ & $55.89^{\circ}$ & $60^{\circ}$ & 1.3385 & 1.4 & $0.5+4 i$ & $99.62 \%$ & $92.0 \%$ & $78.3 \%$ & $99.62 \%$ & $84.54 \%$ \\
\hline 451 & $60^{\circ}$ & $55.79^{\circ}$ & $55.79^{\circ}$ & $60^{\circ}$ & 1.3370 & 1.4 & $0.6+4.3 i$ & $99.61 \%$ & $91.6 \%$ & $77.1 \%$ & $99.61 \%$ & $83.69 \%$ \\
\hline 496 & $60^{\circ}$ & $55.68^{\circ}$ & $55.68^{\circ}$ & $60^{\circ}$ & 1.3352 & 1.4 & $0.75+4.8 \mathrm{i}$ & $99.58 \%$ & $91.4 \%$ & $76.4 \%$ & $99.58 \%$ & $83.19 \%$ \\
\hline 550 & $60^{\circ}$ & $55.55^{\circ}$ & $55.55^{\circ}$ & $60^{\circ}$ & 1.3330 & 1.4 & $0.7+4.7 i$ & $99.56 \%$ & $91.6 \%$ & $77.1 \%$ & $99.56 \%$ & $83.60 \%$ \\
\hline 551 & $60^{\circ}$ & $55.55^{\circ}$ & $55.55^{\circ}$ & $60^{\circ}$ & 1.3330 & 1.4 & $0.85+5.5 i$ & $99.56 \%$ & $92.3 \%$ & $78.6 \%$ & $99.56 \%$ & $84.71 \%$ \\
\hline 600 & $60^{\circ}$ & $55.48^{\circ}$ & $55.48^{\circ}$ & $60^{\circ}$ & 1.3320 & 1.4 & $0.8+5 i$ & $99.55 \%$ & $91.4 \%$ & $76.6 \%$ & $99.55 \%$ & $83.25 \%$ \\
\hline 620 & $60^{\circ}$ & $55.48^{\circ}$ & $55.48^{\circ}$ & $60^{\circ}$ & 1.3320 & 1.4 & $1.05+6 i$ & $99.55 \%$ & $91.9 \%$ & $77.7 \%$ & $99.55 \%$ & $84.05 \%$ \\
\hline 650 & $60^{\circ}$ & $55.42^{\circ}$ & $55.42^{\circ}$ & $60^{\circ}$ & 1.3310 & 1.4 & $0.9+5.42 i$ & $99.54 \%$ & $91.6 \%$ & $77.1 \%$ & $99.54 \%$ & $83.60 \%$ \\
\hline 310 & $70^{\circ}$ & $64.78^{\circ}$ & $64.78^{\circ}$ & $70^{\circ}$ & 1.3478 & 1.4 & $0.38+3 i$ & $98.77 \%$ & $92.7 \%$ & $75.1 \%$ & $98.77 \%$ & $81.84 \%$ \\
\hline 354 & $70^{\circ}$ & $64.32^{\circ}$ & $64.32^{\circ}$ & $70^{\circ}$ & 1.3427 & 1.4 & $0.4+3.4 i$ & $98.57 \%$ & $93.5 \%$ & $76.9 \%$ & $98.57 \%$ & $82.80 \%$ \\
\hline 381 & $70^{\circ}$ & $64.13^{\circ}$ & $64.13^{\circ}$ & $70^{\circ}$ & 1.3405 & 1.4 & $0.45+3.7 i$ & $98.49 \%$ & $93.6 \%$ & $76.6 \%$ & $98.49 \%$ & $82.57 \%$ \\
\hline 413 & $70^{\circ}$ & $63.95^{\circ}$ & $63.95^{\circ}$ & $70^{\circ}$ & 1.3385 & 1.4 & $0.5+4 i$ & $98.41 \%$ & $93.7 \%$ & $76.6 \%$ & $98.41 \%$ & $82.48 \%$ \\
\hline 451 & $70^{\circ}$ & $63.82^{\circ}$ & $63.82^{\circ}$ & $70^{\circ}$ & 1.3370 & 1.4 & $0.6+4.3 i$ & $98.35 \%$ & $93.4 \%$ & $74.9 \%$ & $98.35 \%$ & $81.39 \%$ \\
\hline 496 & $70^{\circ}$ & $63.66^{\circ}$ & $63.66^{\circ}$ & $70^{\circ}$ & 1.3352 & 1.4 & $0.75+4.8 \mathrm{i}$ & $98.27 \%$ & $93.2 \%$ & $73.7 \%$ & $98.27 \%$ & $80.57 \%$ \\
\hline 550 & $70^{\circ}$ & $63.47^{\circ}$ & $63.47^{\circ}$ & $70^{\circ}$ & 1.3330 & 1.4 & $0.7+4.7 i$ & $98.18 \%$ & $93.3 \%$ & $74.5 \%$ & $98.18 \%$ & $80.91 \%$ \\
\hline 551 & $70^{\circ}$ & $63.47^{\circ}$ & $63.47^{\circ}$ & $70^{\circ}$ & 1.3330 & 1.4 & $0.85+5.5 i$ & $98.18 \%$ & $93.9 \%$ & $75.7 \%$ & $98.18 \%$ & $81.73 \%$ \\
\hline 600 & $70^{\circ}$ & $63.39^{\circ}$ & $63.39^{\circ}$ & $70^{\circ}$ & 1.3320 & 1.4 & $0.8+5 i$ & $98.14 \%$ & $93.2 \%$ & $73.8 \%$ & $98.14 \%$ & $80.39 \%$ \\
\hline 620 & $70^{\circ}$ & $63.39^{\circ}$ & $63.39^{\circ}$ & $70^{\circ}$ & 1.3320 & 1.4 & $1.05+6 i$ & $98.14 \%$ & $93.6 \%$ & $74.3 \%$ & $98.14 \%$ & $80.88 \%$ \\
\hline 650 & $70^{\circ}$ & $63.30^{\circ}$ & $63.30^{\circ}$ & $70^{\circ}$ & 1.3310 & 1.4 & $0.9+5.42 i$ & $98.10 \%$ & $93.3 \%$ & $74.0 \%$ & $98.10 \%$ & $80.55 \%$ \\
\hline 310 & $80^{\circ}$ & $71.46^{\circ}$ & $71.46^{\circ}$ & $80^{\circ}$ & 1.3478 & 1.4 & $0.38+3 i$ & $91.35 \%$ & $94.5 \%$ & $76.8 \%$ & $91.35 \%$ & $71.49 \%$ \\
\hline 354 & $80^{\circ}$ & $70.82^{\circ}$ & $70.82^{\circ}$ & $80^{\circ}$ & 1.3427 & 1.4 & $0.4+3.4 \mathrm{i}$ & $90.46 \%$ & $95.1 \%$ & $77.5 \%$ & $90.46 \%$ & $70.62 \%$ \\
\hline 381 & $80^{\circ}$ & $70.56^{\circ}$ & $70.56^{\circ}$ & $80^{\circ}$ & 1.3405 & 1.4 & $0.45+3.7 i$ & $90.09 \%$ & $95.1 \%$ & $76.7 \%$ & $90.09 \%$ & $69.72 \%$ \\
\hline 413 & $80^{\circ}$ & $70.31^{\circ}$ & $70.31^{\circ}$ & $80^{\circ}$ & 1.3385 & 1.4 & $0.5+4 i$ & $89.74 \%$ & $95.2 \%$ & $76.1 \%$ & $89.74 \%$ & $68.99 \%$ \\
\hline 451 & $80^{\circ}$ & $70.13^{\circ}$ & $70.13^{\circ}$ & $80^{\circ}$ & 1.3370 & 1.4 & $0.6+4.3 i$ & $89.49 \%$ & $94.9 \%$ & $74.0 \%$ & $89.49 \%$ & $67.60 \%$ \\
\hline 496 & $80^{\circ}$ & $69.92^{\circ}$ & $69.92^{\circ}$ & $80^{\circ}$ & 1.3352 & 1.4 & $0.75+4.8 i$ & $89.19 \%$ & $94.7 \%$ & $72.0 \%$ & $89.19 \%$ & $66.28 \%$ \\
\hline 550 & $80^{\circ}$ & $69.67^{\circ}$ & $69.67^{\circ}$ & $80^{\circ}$ & 1.3330 & 1.4 & $0.7+4.7 i$ & $88.84 \%$ & $94.8 \%$ & $73.0 \%$ & $88.84 \%$ & $66.19 \%$ \\
\hline 551 & $80^{\circ}$ & $69.67^{\circ}$ & $69.67^{\circ}$ & $80^{\circ}$ & 1.3330 & 1.4 & $0.85+5.5 i$ & $88.84 \%$ & $95.2 \%$ & $73.3 \%$ & $88.84 \%$ & $66.51 \%$ \\
\hline 600 & $80^{\circ}$ & $69.55^{\circ}$ & $69.55^{\circ}$ & $80^{\circ}$ & 1.3320 & 1.4 & $0.8+5 i$ & $88.67 \%$ & $94.6 \%$ & $71.8 \%$ & $88.67 \%$ & $65.43 \%$ \\
\hline 620 & $80^{\circ}$ & $69.55^{\circ}$ & $69.55^{\circ}$ & $80^{\circ}$ & 1.3320 & 1.4 & $1.05+6 i$ & $88.67 \%$ & $95.0 \%$ & $71.5 \%$ & $88.67 \%$ & $65.43 \%$ \\
\hline 650 & $80^{\circ}$ & $69.43^{\circ}$ & $69.43^{\circ}$ & $80^{\circ}$ & 1.3310 & 1.4 & $0.9+5.42 i$ & $88.51 \%$ & $94.8 \%$ & $71.6 \%$ & $88.51 \%$ & $65.19 \%$ \\
\hline 310 & $90^{\circ}$ & $74.30^{\circ}$ & $74.30^{\circ}$ & $90^{\circ}$ & 1.3478 & 1.4 & $0.38+3 i$ & $0.00 \%$ & $95.4 \%$ & $78.3 \%$ & $0.00 \%$ & $0.00 \%$ \\
\hline 354 & $90^{\circ}$ & $73.55^{\circ}$ & $73.55^{\circ}$ & $90^{\circ}$ & 1.3427 & 1.4 & $0.4+3.4 \mathrm{i}$ & $0.00 \%$ & $95.8 \%$ & $78.5 \%$ & $0.00 \%$ & $0.00 \%$ \\
\hline 381 & $90^{\circ}$ & $73.24^{\circ}$ & $73.24^{\circ}$ & $90^{\circ}$ & 1.3405 & 1.4 & $0.45+3.7 i$ & $0.00 \%$ & $95.8 \%$ & $77.4 \%$ & $0.00 \%$ & $0.00 \%$ \\
\hline
\end{tabular}




\begin{tabular}{|c|c|c|c|c|c|c|c|c|c|c|c|c|}
\hline 413 & $90^{\circ}$ & $72.95^{\circ}$ & $72.95^{\circ}$ & $90^{\circ}$ & 1.3385 & 1.4 & $0.5+4 i$ & $0.00 \%$ & $95.8 \%$ & $76.5 \%$ & $0.00 \%$ & $0.00 \%$ \\
\hline 451 & $90^{\circ}$ & $72.74^{\circ}$ & $72.74^{\circ}$ & $90^{\circ}$ & 1.3370 & 1.4 & $0.6+4.3 i$ & $0.00 \%$ & $95.51 \%$ & $74.1 \%$ & $0.00 \%$ & $0.00 \%$ \\
\hline 496 & $90^{\circ}$ & $72.49^{\circ}$ & $72.49^{\circ}$ & $90^{\circ}$ & 1.3352 & 1.4 & $0.75+4.8 \mathrm{i}$ & $0.00 \%$ & $95.3 \%$ & $71.7 \%$ & $0.00 \%$ & $0.00 \%$ \\
\hline 550 & $90^{\circ}$ & $72.20^{\circ}$ & $72.20^{\circ}$ & $90^{\circ}$ & 1.3330 & 1.4 & $0.7+4.7 i$ & $0.00 \%$ & $95.4 \%$ & $72.7 \%$ & $0.00 \%$ & $0.00 \%$ \\
\hline 551 & $90^{\circ}$ & $72.20^{\circ}$ & $72.20^{\circ}$ & $90^{\circ}$ & 1.3330 & 1.4 & $0.85+5.5 i$ & $0.00 \%$ & $95.8 \%$ & $72.6 \%$ & $0.00 \%$ & $0.00 \%$ \\
\hline 600 & $90^{\circ}$ & $72.07^{\circ}$ & $72.07^{\circ}$ & $90^{\circ}$ & 1.3320 & 1.4 & $0.8+5 i$ & $0.00 \%$ & $95.3 \%$ & $71.3 \%$ & $0.00 \%$ & $0.00 \%$ \\
\hline 620 & $90^{\circ}$ & $72.07^{\circ}$ & $72.07^{\circ}$ & $90^{\circ}$ & 1.3320 & 1.4 & $1.05+6 i$ & $0.00 \%$ & $95.6 \%$ & $70.4 \%$ & $0.00 \%$ & $0.00 \%$ \\
\hline 650 & $90^{\circ}$ & $71.94^{\circ}$ & $71.94^{\circ}$ & $90^{\circ}$ & 1.3310 & 1.4 & $0.9+5.42 i$ & $0.00 \%$ & $95.4 \%$ & $70.9 \%$ & $0.00 \%$ & $0.00 \%$ \\
\hline$\frac{2}{3}$ & $\Phi$ & $\begin{array}{l}\Phi \\
\stackrel{\Phi}{\prime \prime} \\
\Phi \\
\Phi \\
\Xi\end{array}$ & 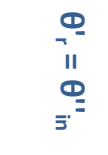 & $\stackrel{\Phi}{=}$ & $\begin{array}{l}\text { ग्र } \\
\text { న్⿱亠䒑 }\end{array}$ & 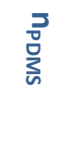 & $\frac{2}{3}$ & 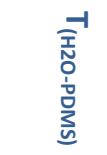 & 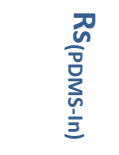 & 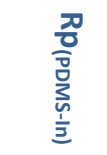 & 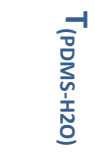 & 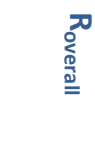 \\
\hline
\end{tabular}

The figures in this table show something quite interesting. In the first place, notice that for an angle of incidence under $80^{\circ}$, reflectivity is over $80 \%$ almost always, which is a pretty good value. To get a better idea of what is displayed in Table 3.2 we plotted the values in the last columns as a function of the incidence angle for a wavelength of $450 \mathrm{~nm}$ (Figure 3.9). It must be said that the reflectivity decay above $80^{\circ}$ of incidence can be fixed and avoided by removing the PDMS gap between the channel and the mirror, achieving then just what is displayed at Figure 3.9.b as the overall reflectivity. Although this measure has not been implemented for the experiments on this thesis, the process is explained in [4] and it is likely to be done in our future work.
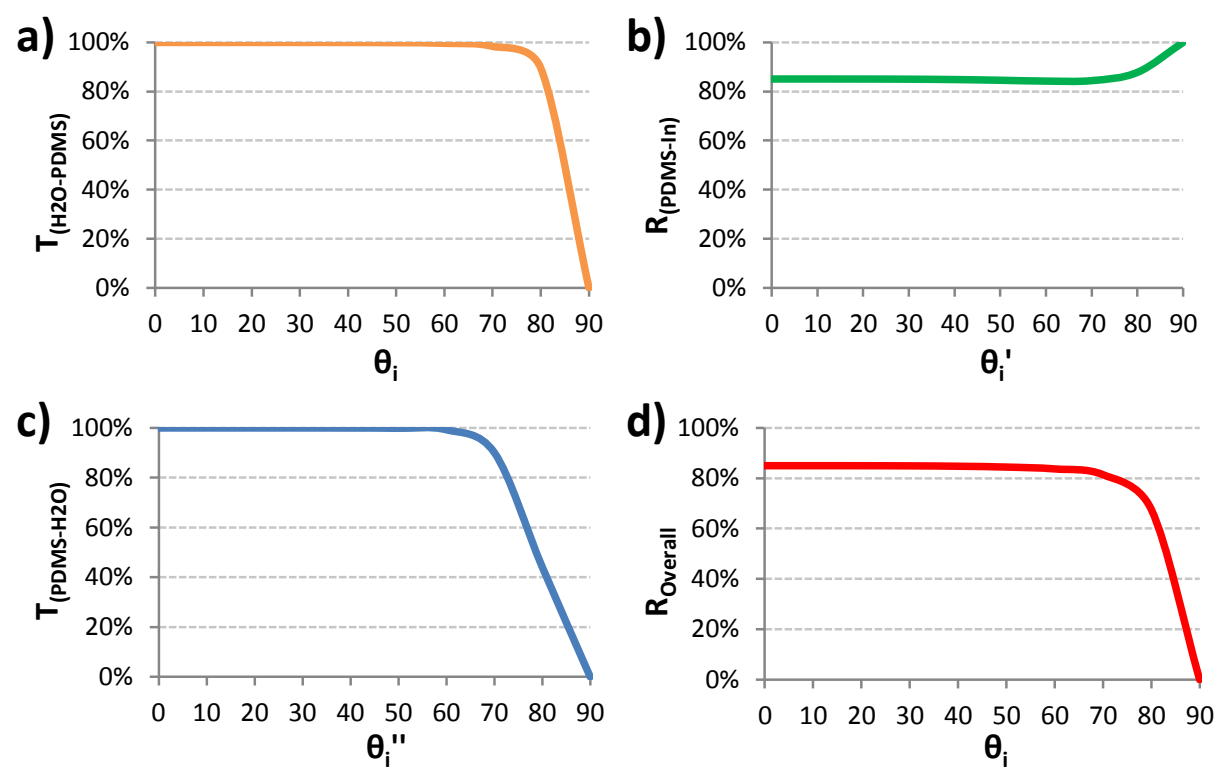

Fig. 3.9 Calculations of (b) transmittance from $\mathrm{H} 2 \mathrm{O}$ to PDMS, (c) reflectance at the PDMS-Indium interface, (d) transmittance from PDMS into water, and (e) overall reflectance-i.e., ratio of incident to reflected light in the aqueous medium. Calculations were performed for a wavelength of $450 \mathrm{~nm}$. 
Another thing in the table to notice about, is that the angle of the final reflected beam is the same as the first incidence angle $\left(\theta_{i}=\theta_{t}{ }^{\prime \prime}\right)$. This is interesting since for a multiple internal reflection (MIR) system with more than one mirror, the final light that is not lost in reflections will just be:

$$
I_{\text {ef }}=I_{0}\left(R_{\text {overall }}\right)^{N}
$$

Eq. 3.1 Amount of light that stays in a N-MIR system and is not lost in the reflection processes.

Being $\mathrm{I}_{0}$ the light introduced in the system, $\mathrm{N}$ the amount of mirrors in the layout and $R_{\text {overall }}$ the total reflectivity from a whole fluid-PDMS-mirror multilayer reflection, or in other words, the light that is not lost in a single reflection process (last column of Table 3.2). It is defined $I_{e f}$ as the useful light that stays in the system to be absorbed by the fluid.

\subsection{Measurement Configuration}

\subsubsection{Setup of the experiment}

All the measurements during this thesis will be taken using the configuration shown in Figure 3.10. Light from the white light source will be injected through an optical fiber into the input of the PDMS device. Another fiber at the output will collect the light back and send it to the spectrometer, which will send the obtained values of the intensity for each wavelength to the computer. Afterwards we will be able to manipulate and correlate all this data to make observations and extract conclusions.

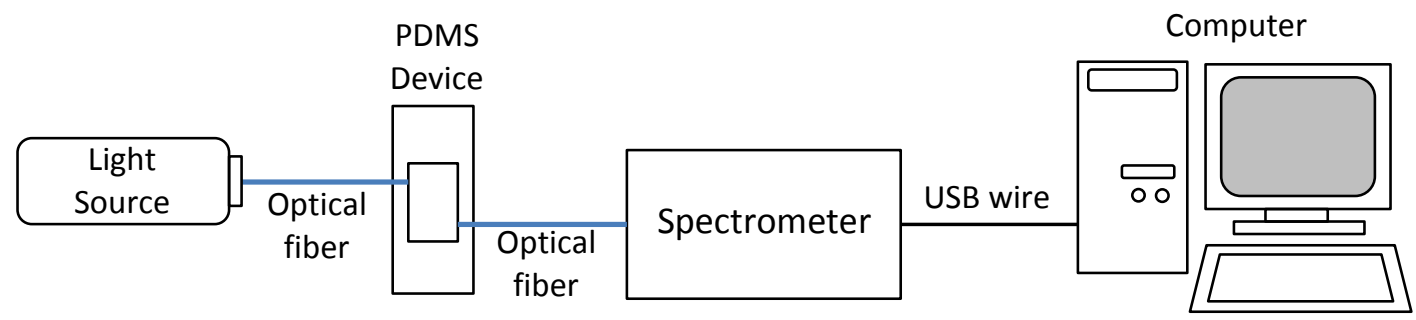

Fig. 3.10 Diagram of the experimental setup to perform measurements with the microfluidic device.

\subsubsection{Instrumentation used}

The instrumentation that we will be using is:

a) White light source: OceanOptics HL-2000-HP-FHSA [10]

b) Spectrometer: OceanOptics HR-4000 [11]

c) Acquisition software: SpectraSuite Spectroscopy Platform [12]

Additionally, while running all the measurements, the device will be mounted in a microscope plate to be able to check at any time the absence of bubbles in the sampling fluid and to plug and unplug the optical fibers into the device without damaging the PDMS (see Figure 3.11). 


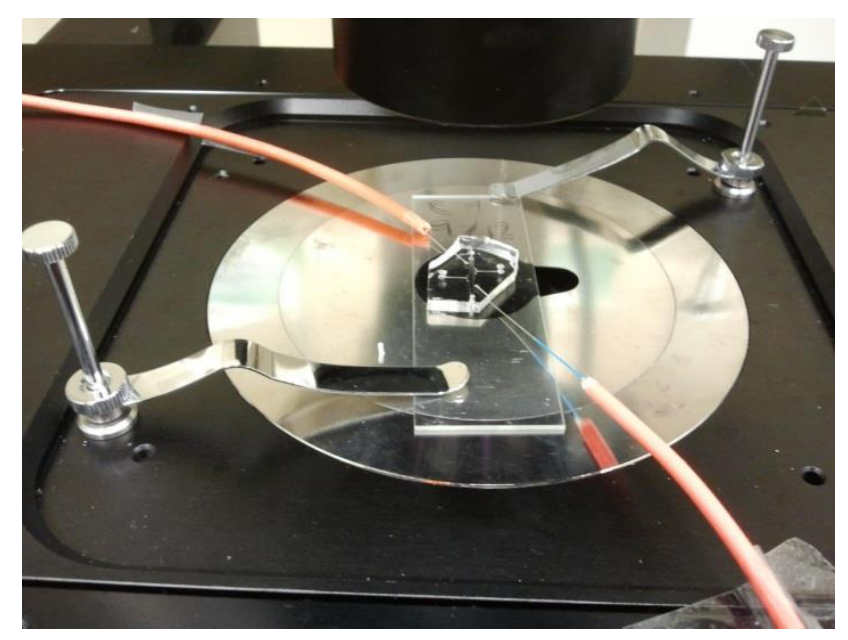

Fig. 3.11 Picture showing the microfluidic device mounted on the microscope platform and the input (top-left) and output (bottom-right) fibers that go to the light source and spectrometer respectively.

\subsection{References}

[1] A. B. Shrirao and R. Perez-Castillejos, "System and method for novel microfluidic device," \#61/414,237.

[2] R. Broto, "Design and Fabrication of Integrated Indium Micromirrors," MS thesis, Universitat Politècnica de Catalunya, Barcelona, 2012.

[3] D. Qin, Y. Xia, and G. M. Whitesides, "Soft lithography for micro and nanoscale patterning," Nature Protocols, vol. 5, pp. 491-502, 2010.

[4] A. B. Shrirao, "High aspect ratio electrodes for high yield electroporation of cells," Ph.D. dissertation, Biomedical Eng., New Jersey Institute of Technology, New Jersey, 2012.

[5] A. Llobera, S. Demming, R. Wilke, and S. Büttgenbach, "Multiple internal reflection poly(dimethylsiloxane) systems for optical sensing," Lab Chip, vol. 7, pp. 1560-1566, 2007.

[6] A. Llobera, R. Wilke, and S. Büttgenbach, "Enhancement of the response of poly(dimethylsiloxane) hollow prisms through air mirrors for absorbance-based sensing," Talanta, vol. 75, pp. 473-479, 2008.

[7] D. V. Vezenov, B. T. Mayers, R. S. Conroy, G. M. Whitesides, P. T. Snee, Y. Chan, D. G. Nocera, and M. G. Bawendi, "A Low-Threshold, High-Efficiency Microfluidic Waveguide Laser," Journal of the Americal Chemical Society, vol. 127, pp. 8952-8953, 2005.

[8] (2013). 2013 U.S. Mineral Commodity Summaries [Online]. Available: http://minerals.usgs.gov/minerals/pubs/mcs/2013/mcs2013.pdf

[9] E. D. Palik, "Introduction to the Data for Several Metals," in Handbook of Optical Constants of Solids. vol. 3, ed San Diego: Academic Press, 1998, pp. 261-268.

[10] OceanOptics. HL-2000 Tungsten Halogen Light Sources [Online]. Available: http://www.oceanoptics.com/products/hl2000.asp

[11] OceanOptics. HR4000 High-Resolution Spectrometer [Online]. Available: http://www.oceanoptics.com/Products/hr4000.asp

[12] OceanOptics. Spectra Suite Spectroscopy Platform [Online]. Available: http://www.oceanoptics.com/Products/spectrasuite.asp 


\section{Chapter 4: Characterization of the Indium Micromirror}

\subsection{Experimental design}

In this chapter we will characterize the fabricated indium mirrors in order to compare the achieved reflectivity values to those calculated in Chapter 3 (Section 3.2.3) for an ideally flat and smooth indium surface. Since due to the design limitations it is impossible to isolate one single mirror from the rest of the components of the device, we need to figure out a way to measure just the reflectivity of the micromirror inside the system, being able to separate it from any other losses introduced by the rest of the elements.

This means that the characterization of the indium will take place inside of the PDMS chip. The first step, then, will be deciding what substance we will put inside the microfluidic channel during the measurements. In order to be able to separate the losses generated by the absorption of the fluid we need it to present a well-known behavior for any incident wavelength in the visible spectrum. It appears that water absorption spectrum has been fully characterized by many research groups [1] and absorbance coefficients for any visible wavelength are available in the literature [2]. We also know that distilled water does not present any fluorescence phenomena[3] or important scattering effects [4]. For all these reasons we choose $\mathrm{H}_{2} \mathrm{O}$ to be the substance present in the microchannel during the measurements to characterize the mirror reflectivity.

In the available literature, we dispose of the water absorbance coefficients $\mu_{a}(\lambda)$ in an e-base exponential format, as shown in Equation 4.1.

$$
\frac{I}{I_{0}}=e^{-\mu_{a}(\lambda) l}
$$

Eq. 4.1 Exponential expression of the absorption of light, being $\mu_{a}(\lambda)$ the absorption coefficient with units of $\mathrm{cm}^{-1}$, at a particular wavelength $(\lambda)$, $I$ the transmitted intensity of light, $I_{0}$ the incident intensity and $l$ the optical path length.

Having that information we design the scenario in which the experiment will take place. For this purpose we need to predict all the losses that the different elements in the system may introduce. This is shown in Figure 4.1.a. The parameter $l$ is the optical path length only through water, which is the same both in the incident and reflected beam. We call $L_{\text {in }}$ and $L_{\text {out }}$ to the transmittance from PDMS to water and from water to PDMS that take place in the input and in the output, respectively, together with all the possible insertion losses. Since we do not know the value of these losses - we can make a prediction for the transmittances but not for all the rest of insertion losses-, we need to repeat the experiment in the same input/output conditions but without a mirror. This way we will be able to separate the light lost in the reflection from the light lost in the insertion/reception ports.

This means that we need to design and fabricate another device that just injects light at one side of the microchannel and collects it at the other side (Figure 4.1.b). Thus, we will get a reading just with the losses introduced by the input and output (and the well known 
absorption of water), and we will be able to compare it to the measurements taken with a one-mirror device, which include the input/output and the reflection losses. The result of this comparison will give us the value of the reflectivity of an indium mirror.

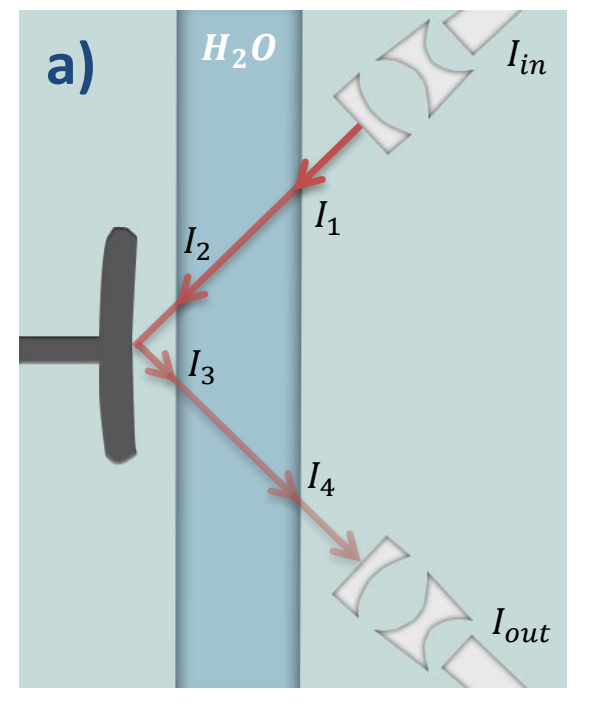

$$
\begin{aligned}
& I_{1}=I_{\text {in }} L_{\text {in }} \\
& I_{2}=I_{\text {in }} L_{\text {in }} e^{-\mu_{a} l} \\
& I_{3}=I_{\text {in }} L_{\text {in }} e^{-\mu_{a} l} R_{m} \\
& I_{4}=I_{\text {in }} L_{\text {in }} e^{-2 \mu_{a} l} R_{m} \\
& I_{\text {out }}=I_{\text {in }} L_{\text {in }} e^{-2 \mu_{a} l} R_{m} L_{\text {out }}
\end{aligned}
$$

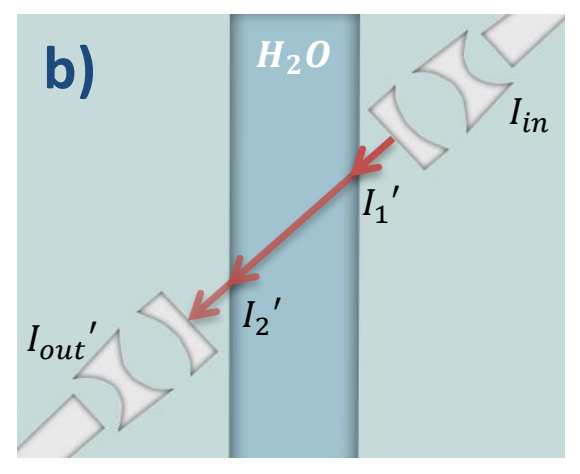

$$
\begin{aligned}
& I_{1}{ }^{\prime}=I_{\text {in }} L_{\text {in }} \\
& I_{2}{ }^{\prime}=I_{\text {in }} L_{\text {in }} e^{-\mu_{a} l^{\prime}} \\
& I_{\text {out }}{ }^{\prime}=I_{\text {in }} L_{\text {in }} e^{-\mu_{a} l^{\prime}} L_{\text {out }}
\end{aligned}
$$

Fig. 4.1 Diagram indicating all the losses of light intensity that take place inside a (a) single-mirror microfluidic device and (b) a no-mirror microfluidic device.

Note that the values $l$ and $l^{\prime}$ in figure 4.1.a and 4.1.b are not the same length. This is mainly because the device without mirrors was fabricated with a wider channel, but it is not a problem since we can calculate the two different values for water absorption. A microscope top view of the two devices that were fabricated to perform these tests can be seen in Figure 4.2. See that to perform the measurements for the single-mirror optical path we are using a chip that correspond to the layout of case number 8 (see Chapter 3, Section 3.1.3). 


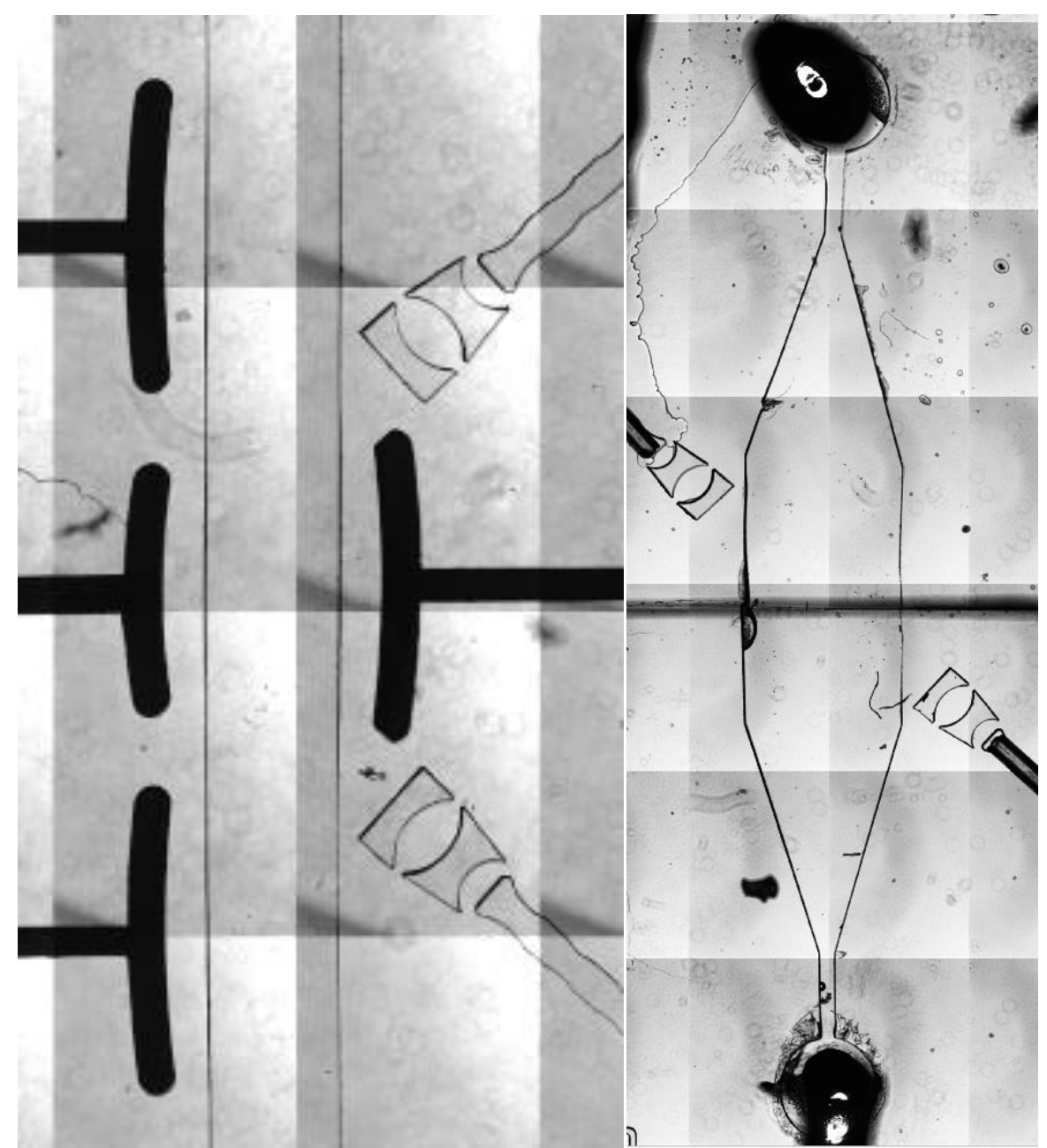

(a)

(b)

Fig. 4.2 Microscope top view of a real fabricated (a) single-mirror microfluidic device and (b) no-mirror microfluidic device.

Taking into account the expressions of the intensity shown in Figure 4.1 we can now design an experiment that allows us to extract from the measurements of these two chips the reflectivity of an indium micromirror. This can be done by calculating the quotient between the results obtained with both devices, as shown in equation 4.2.

$$
\frac{I_{\text {out }}}{I_{\text {out }}{ }^{\prime}}=\frac{I_{\text {in }} L_{\text {in }} e^{-2 \mu_{a} l} R_{m} L_{\text {out }}}{I_{\text {in }} L_{\text {in }} e^{-\mu_{a} l^{\prime}} L_{\text {out }}}=\frac{e^{-2 \mu_{a} l} R_{m}}{e^{-\mu_{a} l^{\prime}}}=e^{-\mu_{a}\left(2 l-l^{\prime}\right)} R_{m}
$$

Eq. 4.2 Quotient between the theoretical intensity of light received in a single-mirror microfluidic device and a no-mirror microfluidic device. As can be seen, the final term only depends on the difference between the two optical path lengths, $\mu_{a}(\lambda)$-which is the absorption coefficient for water with units of $\mathrm{cm}^{-1}$ - and the reflectivity of the indium mirror $R_{m}$. 


\subsection{Experimental Results}

We measured the light received from a microfluidic device with one mirror and another without mirrors for the visible spectrum ( 400 to $1000 \mathrm{~nm}$ ) and with the same input intensity for both cases. The results obtained are plotted in Figure 4.3.a. Then we obtained the quotient between both results $\left(I_{\text {single-mirror }} / I_{\text {no-mirror }}\right)$, which is displayed in Figure 4.3.b.
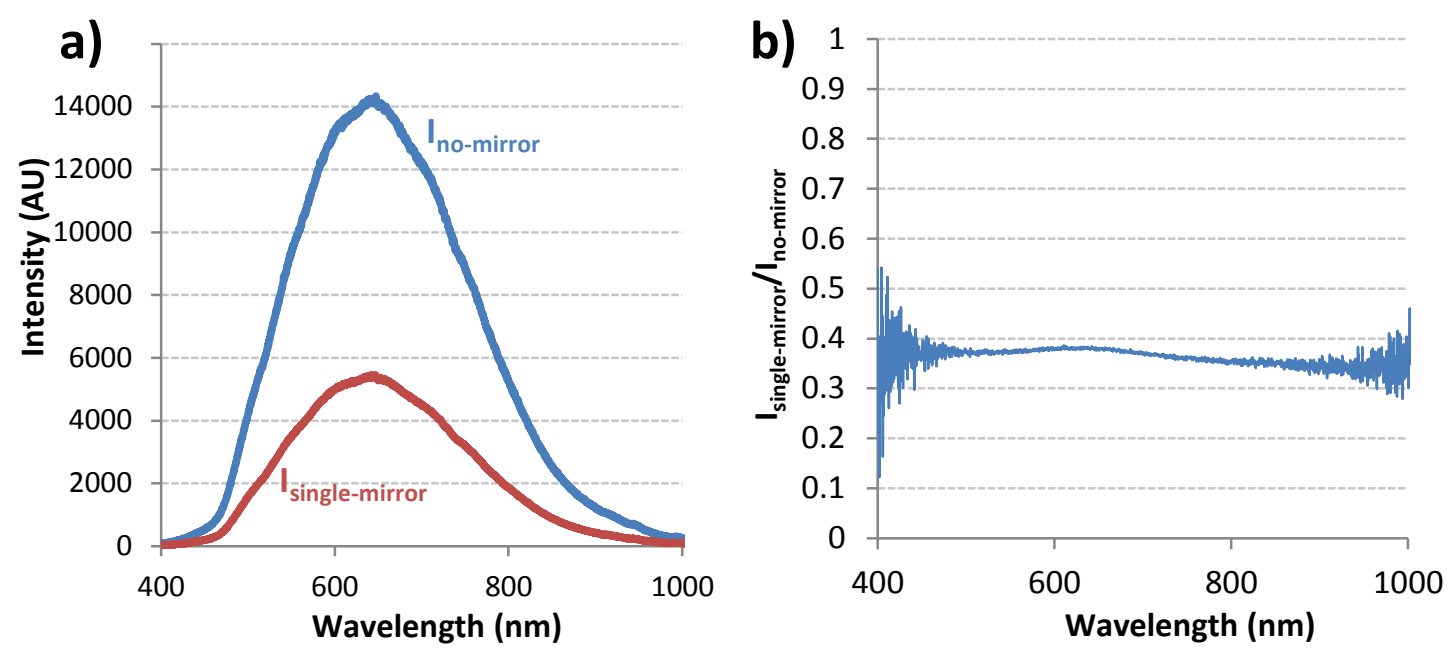

Fig. 4.3 (a) Plot of the received intensity from a single-mirror and a no-mirror microfluidic device as a function of the wavelength. (b) Quotient of the values displayed in (a).

As can be seen in Equation 4.2, the values displayed in Figure 4.3.b -which are compressed between 0.31 and 0.39 - are a function of the absorbance of water (which depends on $\lambda$ and the path length) and the reflectivity of an indium micromirror. The mean value of the curve during the interval for valid data -from 500 to $850 \mathrm{~nm}$, since there is too much noise for the rest of the spectrum- is $36.8 \%$. This is the average loss generated by our mirror and by water absorption. Thus, if we divide the values plotted in Figure 4.3.b by the attenuation generated by the water, we will get the target value of the mirror reflectivity as a function of wavelength.

Next step, then, is calculating the value of $e^{-\mu_{a}\left(2 l-l^{\prime}\right)}$ that Equation 4.2 defines. As the singlemirror device corresponds to a configuration of the case 8 layout explained in Chapter 3 (Section 3.1.3), we can get from the fifth column of Table 3.1 that $2 l=2470 \mu \mathrm{m}$. The value of $l^{\prime}$ was measured with the microscope software, obtaining a length of $2460 \mu \mathrm{m}$. Then, $2 l-l^{\prime}=10 \mu \mathrm{m}=1 \cdot 10^{-3} \mathrm{~cm}$ (since the units of $\mu_{a}$ are $\left.\mathrm{cm}^{-1}\right)$.

We calculated then $e^{-\mu_{a}(\lambda) 10^{-3}}$ for all the values available in [2], and placed them in Table 4.1. It can be observed that if we round the values to five significant figures, we will always get 0.99999, which is negligible in front of the losses measured in our experiment. So we can conclude that the values plotted in Figure 4.3.b (which correspond to $I_{\text {single-mirror }} /$ $I_{n o-m i r r o r}$ ) are actually the real value of the reflectivity of one indium micromirror. 
Table 4.1

Tabulated values for the water absorbance coefficients in $\mathrm{cm}^{-1}\left(2^{\text {nd }}\right.$ column $)$ and the corresponding calculation of the absorbed percentage of light absorbed in a single-mirror device divided by the percentage absorbed in a no-mirror device ( $3^{\text {rd }}$ column).

\begin{tabular}{|c|c|c|}
\hline$\lambda(\mathrm{nm})$ & $-\mu_{a}\left(\mathrm{~cm}^{-1}\right)$ & $e^{-\mu_{a}(\lambda) 10^{-3}}$ \\
\hline 400 & $6.63 \mathrm{E}-05$ & 0.99999993 \\
\hline 402.5 & 5.79E-05 & 0.99999994 \\
\hline 405 & 5.30E-05 & 0.99999995 \\
\hline 407.5 & 5.03E-05 & 0.99999995 \\
\hline 410 & 4.73E-05 & 0.99999995 \\
\hline 412.5 & 4.52E-05 & 0.99999995 \\
\hline 415 & 4.44E-05 & 0.99999996 \\
\hline 417.5 & 4.42E-05 & 0.99999996 \\
\hline 420 & 4.54E-05 & 0.99999995 \\
\hline 422.5 & 4.74E-05 & 0.99999995 \\
\hline 425 & 4.78E-05 & 0.99999995 \\
\hline 427.5 & $4.82 \mathrm{E}-05$ & 0.99999995 \\
\hline 430 & 4.95E-05 & 0.99999995 \\
\hline 432.5 & 5.04E-05 & 0.99999995 \\
\hline 435 & 5.30E-05 & 0.99999995 \\
\hline 437.5 & 5.80E-05 & 0.99999994 \\
\hline 440 & $6.35 \mathrm{E}-05$ & 0.99999994 \\
\hline 442.5 & $6.96 \mathrm{E}-05$ & 0.99999993 \\
\hline 445 & 7.51E-05 & 0.99999992 \\
\hline 447.5 & 8.30E-05 & 0.99999992 \\
\hline 450 & $9.22 \mathrm{E}-05$ & 0.99999991 \\
\hline 452.5 & 9.69E-05 & 0.99999990 \\
\hline 455 & $9.62 \mathrm{E}-05$ & 0.99999990 \\
\hline 457.5 & 9.57E-05 & 0.99999990 \\
\hline 460 & 9.79E-05 & 0.99999990 \\
\hline 462.5 & 1.01E-04 & 0.99999990 \\
\hline 465 & 1.01E-04 & 0.99999990 \\
\hline 467.5 & $1.02 \mathrm{E}-04$ & 0.99999990 \\
\hline 470 & 1.06E-04 & 0.99999989 \\
\hline 472.5 & 1.09E-04 & 0.99999989 \\
\hline 475 & $1.14 \mathrm{E}-04$ & 0.99999989 \\
\hline 477.5 & $1.21 \mathrm{E}-04$ & 0.99999988 \\
\hline 480 & 1.27E-04 & 0.99999987 \\
\hline 482.5 & 1.31E-04 & 0.99999987 \\
\hline 485 & $1.36 \mathrm{E}-04$ & 0.99999986 \\
\hline 487.5 & $1.44 \mathrm{E}-04$ & 0.99999986 \\
\hline 490 & $1.50 \mathrm{E}-04$ & 0.99999985 \\
\hline 492.5 & $1.62 \mathrm{E}-04$ & 0.99999984 \\
\hline 495 & 1.73E-04 & 0.99999983 \\
\hline 497.5 & 1.91E-04 & 0.99999981 \\
\hline
\end{tabular}

\begin{tabular}{|c|c|c|}
\hline$\lambda(\mathrm{nm})$ & $-\mu_{a}\left(\mathrm{~cm}^{-1}\right)$ & $e^{-\mu_{a}(\lambda) 10^{-3}}$ \\
\hline 500 & 2.04E-04 & 0.99999980 \\
\hline 502.5 & $2.28 \mathrm{E}-04$ & 0.99999977 \\
\hline 505 & $2.56 \mathrm{E}-04$ & 0.99999974 \\
\hline 507.5 & 2.80E-04 & 0.99999972 \\
\hline 510 & $3.25 \mathrm{E}-04$ & 0.99999968 \\
\hline 512.5 & 3.72E-04 & 0.99999963 \\
\hline 515 & 3.96E-04 & 0.99999960 \\
\hline 517.5 & 3.99E-04 & 0.99999960 \\
\hline 520 & 4.09E-04 & 0.99999959 \\
\hline 522.5 & 4.16E-04 & 0.99999958 \\
\hline 525 & 4.17E-04 & 0.99999958 \\
\hline 527.5 & 4.28E-04 & 0.99999957 \\
\hline 530 & 4.34E-04 & 0.99999957 \\
\hline 532.5 & 4.47E-04 & 0.99999955 \\
\hline 535 & 4.52E-04 & 0.99999955 \\
\hline 537.5 & 4.66E-04 & 0.99999953 \\
\hline 540 & 4.74E-04 & 0.99999953 \\
\hline 542.5 & 4.89E-04 & 0.99999951 \\
\hline 545 & 5.11E-04 & 0.99999949 \\
\hline 547.5 & 5.37E-04 & 0.99999946 \\
\hline 550 & 5.65E-04 & 0.99999944 \\
\hline 552.5 & 5.93E-04 & 0.99999941 \\
\hline 555 & 5.96E-04 & 0.99999940 \\
\hline 557.5 & $6.06 \mathrm{E}-04$ & 0.99999939 \\
\hline 560 & $6.19 \mathrm{E}-04$ & 0.99999938 \\
\hline 562.5 & $6.40 \mathrm{E}-04$ & 0.99999936 \\
\hline 565 & $6.42 \mathrm{E}-04$ & 0.99999936 \\
\hline 567.5 & $6.72 \mathrm{E}-04$ & 0.99999933 \\
\hline 570 & $6.95 \mathrm{E}-04$ & 0.99999931 \\
\hline 572.5 & 7.33E-04 & 0.99999927 \\
\hline 575 & $7.72 \mathrm{E}-04$ & 0.99999923 \\
\hline 577.5 & 8.36E-04 & 0.99999916 \\
\hline 580 & 8.96E-04 & 0.99999910 \\
\hline 582.5 & 9.89E-04 & 0.99999901 \\
\hline 585 & 1.10E-03 & 0.99999890 \\
\hline 587.5 & $1.22 \mathrm{E}-03$ & 0.99999878 \\
\hline 590 & $1.35 \mathrm{E}-03$ & 0.99999865 \\
\hline 592.5 & $1.52 \mathrm{E}-03$ & 0.99999848 \\
\hline 595 & 1.67E-03 & 0.99999833 \\
\hline 597.5 & 1.93E-03 & 0.99999808 \\
\hline
\end{tabular}




\begin{tabular}{|c|c|c|}
\hline$\lambda(\mathrm{nm})$ & $-\mu_{a}\left(\mathrm{~cm}^{-1}\right)$ & $e^{-\mu_{a}(\lambda) 10^{-3}}$ \\
\hline 600 & $2.22 \mathrm{E}-03$ & 0.99999778 \\
\hline 602.5 & $2.47 \mathrm{E}-03$ & 0.99999753 \\
\hline 605 & $2.58 \mathrm{E}-03$ & 0.99999742 \\
\hline 607.5 & 2.63E-03 & 0.99999737 \\
\hline 610 & 2.64E-03 & 0.99999736 \\
\hline 612.5 & 2.67E-03 & 0.99999734 \\
\hline 615 & $2.68 \mathrm{E}-03$ & 0.99999732 \\
\hline 617.5 & 2.71E-03 & 0.99999729 \\
\hline 620 & $2.76 \mathrm{E}-03$ & 0.99999725 \\
\hline 622.5 & 2.81E-03 & 0.99999719 \\
\hline 625 & $2.83 \mathrm{E}-03$ & 0.99999717 \\
\hline 627.5 & 2.90E-03 & 0.99999710 \\
\hline 630 & 2.92E-03 & 0.99999708 \\
\hline 632.5 & 3.00E-03 & 0.99999701 \\
\hline 635 & 3.01E-03 & 0.99999699 \\
\hline 637.5 & $3.08 \mathrm{E}-03$ & 0.99999692 \\
\hline 640 & $3.11 \mathrm{E}-03$ & 0.99999689 \\
\hline 642.5 & $3.22 \mathrm{E}-03$ & 0.99999678 \\
\hline 645 & $3.25 \mathrm{E}-03$ & 0.99999675 \\
\hline 647.5 & $3.35 \mathrm{E}-03$ & 0.99999665 \\
\hline 650 & $3.40 \mathrm{E}-03$ & 0.99999660 \\
\hline 652.5 & $3.58 \mathrm{E}-03$ & 0.99999642 \\
\hline 655 & 3.71E-03 & 0.99999629 \\
\hline 657.5 & 3.93E-03 & 0.99999607 \\
\hline 660 & 4.10E-03 & 0.99999590 \\
\hline 662.5 & 4.24E-03 & 0.99999576 \\
\hline 665 & 4.29E-03 & 0.99999571 \\
\hline
\end{tabular}

\begin{tabular}{rrr}
\hline $\boldsymbol{\lambda}(\mathrm{nm})$ & $-\boldsymbol{\mu}_{\boldsymbol{a}}\left(\mathrm{cm}^{-\mathbf{1}}\right)$ & $\boldsymbol{e}^{-\boldsymbol{\mu}_{\boldsymbol{a}}(\boldsymbol{\lambda}) \mathbf{1 0}^{-3}}$ \\
\hline $\mathbf{6 6 7 . 5}$ & $4.36 \mathrm{E}-03$ & 0.99999564 \\
$\mathbf{6 7 0}$ & $4.39 \mathrm{E}-03$ & 0.99999561 \\
$\mathbf{6 7 2 . 5}$ & $4.48 \mathrm{E}-03$ & 0.99999552 \\
$\mathbf{6 7 5}$ & $4.48 \mathrm{E}-03$ & 0.99999552 \\
$\mathbf{6 7 7 . 5}$ & $4.61 \mathrm{E}-03$ & 0.99999539 \\
$\mathbf{6 8 0}$ & $4.65 \mathrm{E}-03$ & 0.99999535 \\
$\mathbf{6 8 2 . 5}$ & $4.78 \mathrm{E}-03$ & 0.99999522 \\
$\mathbf{6 8 5}$ & $4.86 \mathrm{E}-03$ & 0.99999514 \\
$\mathbf{6 8 7 . 5}$ & $5.02 \mathrm{E}-03$ & 0.99999498 \\
$\mathbf{6 9 0}$ & $5.16 \mathrm{E}-03$ & 0.99999484 \\
$\mathbf{6 9 2 . 5}$ & $5.38 \mathrm{E}-03$ & 0.99999462 \\
$\mathbf{6 9 5}$ & $5.59 \mathrm{E}-03$ & 0.99999441 \\
\hline $\mathbf{6 9 7 . 5}$ & $5.92 \mathrm{E}-03$ & 0.99999408 \\
$\mathbf{7 0 0}$ & $6.24 \mathrm{E}-03$ & 0.99999376 \\
\hline $\mathbf{7 0 2 . 5}$ & $6.63 \mathrm{E}-03$ & 0.99999337 \\
$\mathbf{7 0 5}$ & $7.04 \mathrm{E}-03$ & 0.99999296 \\
$\mathbf{7 0 7 . 5}$ & $7.56 \mathrm{E}-03$ & 0.99999244 \\
$\mathbf{7 1 0}$ & $8.27 \mathrm{E}-03$ & 0.99999173 \\
$\mathbf{7 1 2 . 5}$ & $9.14 \mathrm{E}-03$ & 0.99999086 \\
$\mathbf{7 1 5}$ & $1.01 \mathrm{E}-02$ & 0.99998993 \\
$\mathbf{7 1 7 . 5}$ & $1.12 \mathrm{E}-02$ & 0.99998881 \\
$\mathbf{7 2 0}$ & $1.23 \mathrm{E}-02$ & 0.99998769 \\
$\mathbf{7 2 2 . 5}$ & $1.36 \mathrm{E}-02$ & 0.99998644 \\
\hline & $1.49 \mathrm{E}-02$ & 0.99998511 \\
\hline $\mathbf{7 2 5}$ & $1.68 \mathrm{E}-02$ & 0.99998322 \\
\hline
\end{tabular}

\subsection{Discussion}

If we compare the experimental reflectivity of the mirrors ( $37 \%)$ to the theoretical one ( $83 \%)$, we actually find out that there is a very big discrepancy, and that we are losing in the reflection a $55 \%$ of the light that was supposed to be reflected back according to our calculations (see Equation 4.3).

$$
1-\frac{R_{\text {measured }}}{R_{\text {calculated }}}=1-\frac{0.37}{0.83}=55 \%
$$

Eq. 4.3 Calculation for the discrepancy between the theoretical and measured value of the reflectivity of an indium micromirror. That $55 \%$ is the amount of light that is not supposed to be absorbed by the mirror and that is lost elsewhere.

That loss of the $55 \%$ of expected light can be due to several things. It has to be said that the theoretical calculations were made for infinite perfectly smooth and flat indium and PDMS interfaces, and without taking into account the dispersion of the light beam. If we introduce 
real conditions into the picture -namely, the mirrors are not perfectly flat, they are not infinite, the light can disperse during its propagation through the fluid...- we can identify two main causes for our losses.

a) All the light is not hitting the mirror: The mirror can be misplaced or it might not be big enough, or the light beam can be too wide due to dispersion to fit in the mirror.

b) The mirror is not ideal: Due to the limitations of the fabrication methods the mirror has imperfections and a surface that is not flat and smooth, and can cause a shift in the practical reflectivity from its theoretical value.

To check the impact of cause $A$-defined above- in the measured value of the reflectivity we will run a quick experiment that will allow us to observe the optical path across the chip and its shape and behavior.

\subsubsection{Ray tracing test}

Herein we will run a test to check if the indium micromirrors behave as expected, if the light follows the designed path [5] and what shape has the beam -i.e. the widening due to dispersion-. In order to do this we will need to choose a suitable substance to be in the microfluidic channel of the device. With a non-fluorescent molecule we will not be able to see the optical path from a top view of the device, since all the light is directed towards the mirrors and the output, and none upwards (see Figure 4.4.a). That is why we decided to use a $1 \mathrm{mM}$ fluorescein aqueous solution, which has an excitation band in the blue region (around $490 \mathrm{~nm}$ ) and an emission band around $520 \mathrm{~nm}$. This phenomenon will allow us to see the illuminated light path thanks to the isotropic radiation of fluorescein (Figure 4.4.b).

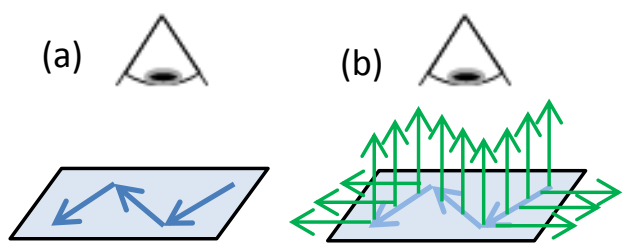

Fig. 4.4 Schematic that shows the need of a fluorescent molecule to be able to see the optical path from a top view of the device. (a) Non-fluorescent fluid; (b) fluorescent fluid.

The results of this experiment can be seen in the picture taken by the microscope shown in Figure 4.5. The device used corresponds to a case 1 layout, which was used in order to be able to see more than one reflection. It can be observed that the light reflects in the indium mirrors as expected, and follows the right optical path. This means that the mirrors have the correct shape and positioning. However, we can see that due to the widening of the light beam caused by dispersion, there is a significant amount of light that does not hit the mirror. 

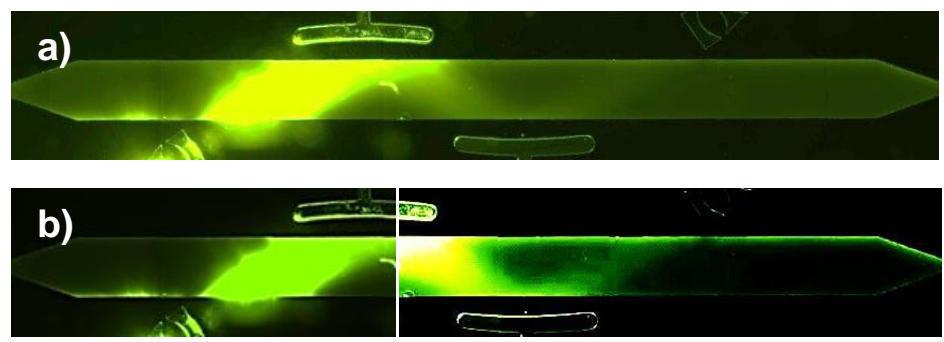

Fig. 4.5 (a) Top view of the microfluidic device filled with a 1 $\mathrm{mM}$ fluorescein aqueous solution while injecting broadband light via the optical input. (b) As an aid to eye, the contrast was enhanced in the right side of the image.

To get an approximation on the quantity of light that falls out of the mirror we used image processing software with the picture in Figure 4.5 to measure the width of the light beam -in pixels-and then we calculated the percentage that overflows the mirror. Figure 4.6 shows how we obtained the width of the beam and how we measured the relative amount of light that was not hitting the mirror. The results in pixels are also displayed in the image.

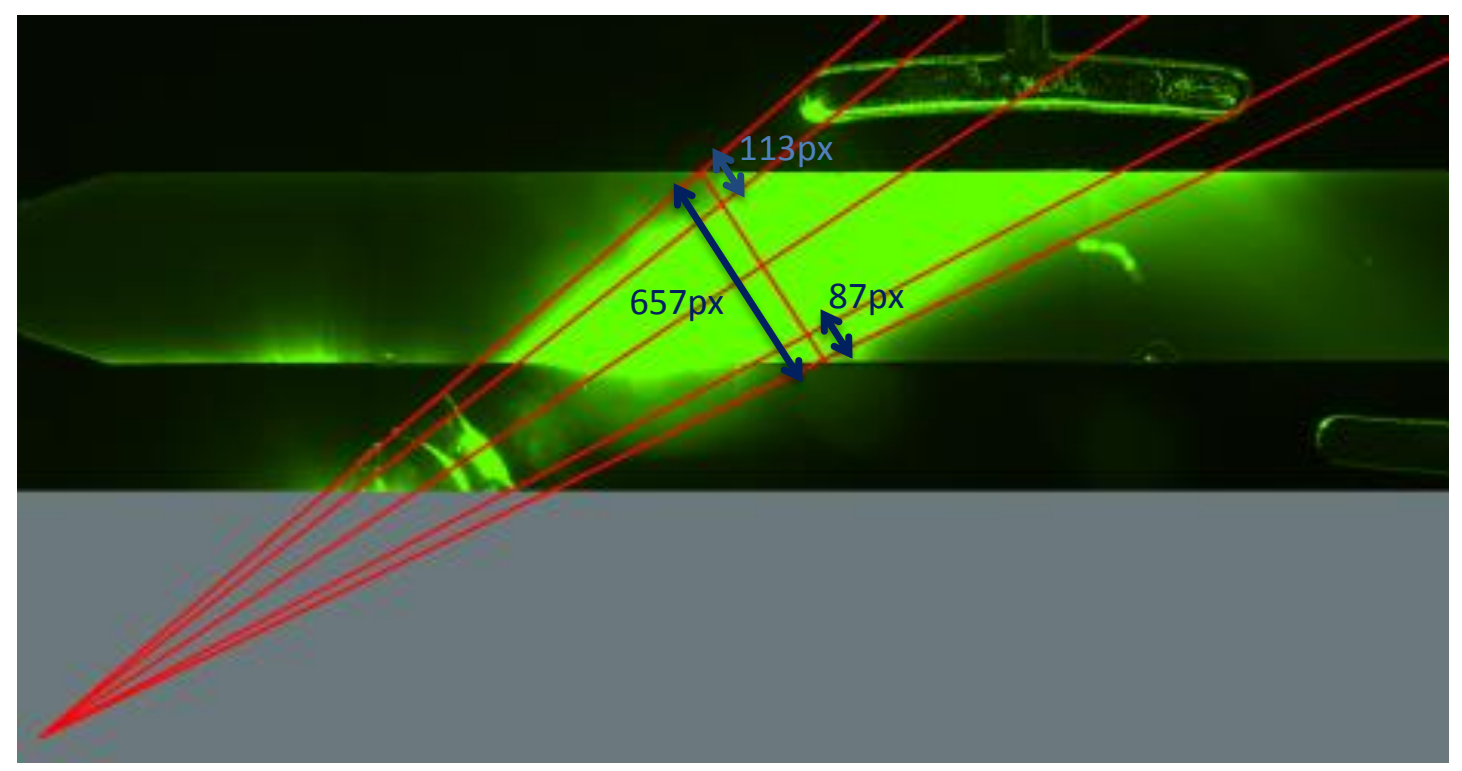

Fig. 4.6 Measurements taken to the picture of the incident light beam performed with image processing software. All the values were measured upon a perpendicular section of the conical light beam and the fractions were found by tracing straight lines that converge in the cone vertex.

It has to be said that this is not intended to be an accurate measurement since we are not taking into account the Gaussian behavior of the intensity of light as the radius of the beam increases. With this experiment we are just trying to get an approximate idea of the light that is being wasted. So, after making all the calculations (Equation 4.3), we find out that about a $30 \%$ of the emitted light is overflowing the mirror. 
$\frac{\text { Overflowing width }}{\text { Total width }}=\frac{113 p x+87 p x}{657 p x}=30.44 \%$

Eq. 4.3 Calculation of the fraction of the emitted light that does not hit de mirror due to the widening of the beam, according to the measurements in Figure 4.6.

According to these results, we can tell that at least about $1 / 3$ of the losses are due to the factor that the beam is wider than the mirror at the moment of the reflection. With this rough measurement we cannot give a numerical value for the real reflectivity $\left(\boldsymbol{R}_{\text {real }}=\boldsymbol{R}_{\text {measured }} \cdot\right.$ Losses $)$ that could be taken into account seriously. To have a more accurate value of the amount of light lost for this reason we would need to know the function of the light intensity as we move away from the center of the beam.

So far, the only think we can tell is that the major part of the discrepancy between the expected and the obtained reflectivity value is because a considerable amount of light coming out from the input is not hitting our mirror. To fix this issue that is causing so many losses in our overall reflectivity we would need a lens in our optical input able to focus light in the center of the mirror.

\subsection{Conclusions}

The real reflectivity of an indium micromirror should be by far over $0.4-45 \%$ of the theoretical value-, but once integrated in the microfluidic device it becomes 0.37 due to the light that does not hit the mirror because of widening of the beam (dispersion).

The final conclusion is that the mirror itself appears to have a good reflectivity. However, we need a better lens if we want to get a more accurate value of this parameter and, of course, if we want to take advantage of all the injected light in the system when performing absorbance measurements with the device. The current lens is intended to focus all the light at the input into parallel beams. A better design of it would focus the light to converge some point near the mirror, so none is lost due to dispersion across the fluid.

\subsection{References}

[1] (1998). Optical Absorption of Water Compendium [Online]. Available: http://omlc.ogi.edu/spectra/water/abs/index.html

[2] R. M. Pope and E. S. Fry, "Absorption spectrum (380 - $700 \mathrm{~nm}$ ) ofpure water. II. Integrating cavity measurements," Applied Optics, vol. 36, pp. 8710-8723, 1997.

[3] V. Drozdowska, S. Babichenko, and A. Lisin, "Natural water fluorescence characteristics based on lidar investigations of a surface water layer polluted by an oil film; the Baltic cruise - May 2000," Oceanologia, vol. 44, pp. 339-354, 2002.

[4] J. E. Tyler, "Scattering properties of distilled and natural waters.," Limnology and Oceanography, vol. 6, pp. 451-456, 1961. 
[5] R. Broto, "Design and Fabrication of Integrated Indium Micromirrors," MS thesis, Universitat Politècnica de Catalunya, Barcelona, 2012. 


\section{Chapter 5: Absorbance Measurements}

\subsection{Experimental design}

After having characterized the indium mirrors in our microfluidic device and proved that they work, the next step is to test if the device itself can be used for its purpose -i.e. if the chip can perform absorbance measurements correctly-. In this chapter we use our microfluidic device to measure the absorbance of light through diverse aqueous fluorescein dilutions and we check if the Beer-Lambert law is followed as it would be expected.

\subsubsection{Selection of the device layout}

For this test, once more we use the simplest configuration that we have, which is the singlereflection one, integrated in the layout of case number 8 (see Chapter 3, Section 3.1.3). This means that the optical path length (present in the equation of the Beer-Lambert's law) will be $2470 \mu \mathrm{m}$.

\subsubsection{Selection of the analyte}

Since this are not real absorbance measurements, but a test to check if the device works properly we decide to use fluorescein as the sampling fluid. This choice was made in order to be able to see the optical path from a top view of the chip while running the measurements, as explained in Chapter 4 (Section 4.3.1). This is interesting since it will allow us to check that at every time the optical path takes the expected form and no deviations happen due to air bubbles, particles of dust or damage in the PDMS (that can happen when plugging/unplugging optical fibers or injecting the analyte). Fluorescein will also help us to detect possible microbubbles or particles that cause scattering, since they will appear notably brighter than the rest of the optical path.

\subsubsection{Spectral band}

As said in Chapter 3 (Section 3.3.2), we are going to be using the OceanOptics HL-2000-HPFHSA white light lamp [1], which has a range of emission from 300 to $1000 \mathrm{~nm}$ approximately, with a maximum around $600 \mathrm{~nm}$.

However, we are not going to be able to make use of this whole spectral region since fluorescein is our analyte and it has an excitation band around $490 \mathrm{~nm}$ and an emission band around 520nm [2]. Above these wavelengths the variations on the received light intensity are not only due to absorption, but also to excitation (which causes an additional attenuation) and emission (which causes an amplification of the received light) of the fluorescein molecules (see Figure 5.1). This results in a function that is hard to predict, since as concentration increases, emission of the molecule will push the intensity up while absorbance of light will pull it down.

Thus, although our light source has a wider emission spectrum, our range of operation will never exceed the $480 \mathrm{~nm}$, being our interest spectral band the region between 400 -since the signal level at $300 \mathrm{~nm}$ is too low- and $480 \mathrm{~nm}$ (Figure 5.1). 


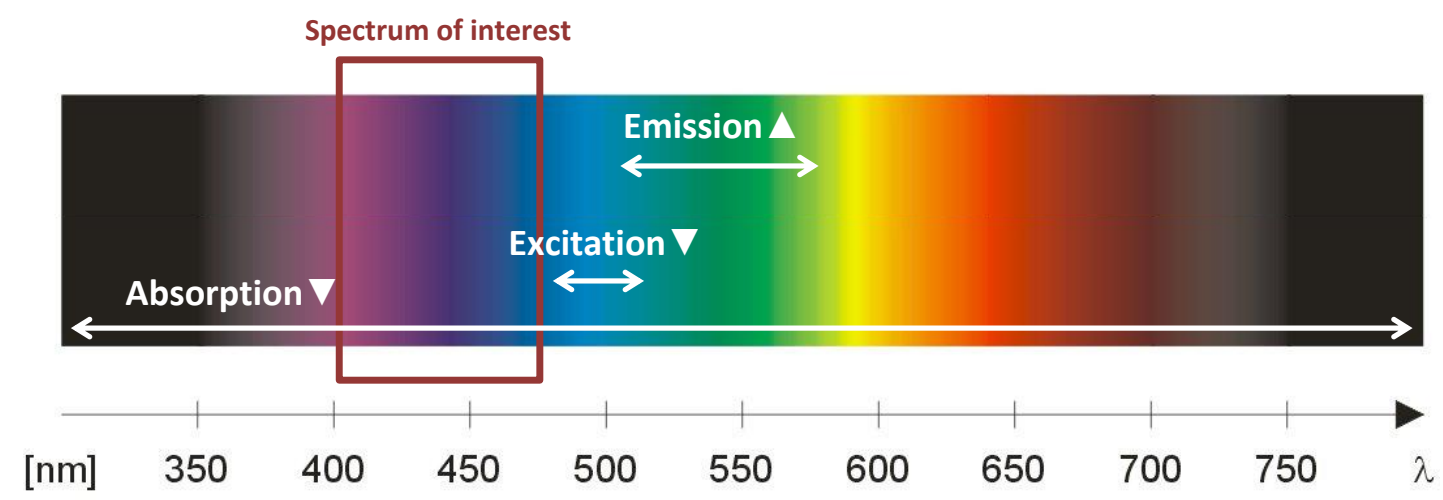

Fig. 5.1 Visible spectrum with the emission, absorption and excitation bands of fluorescein and their influence in the received intensity (marked with triangles that point down if the effect is attenuation and up if it is amplification). It also displays the wavelength range in which we are going to operate to avoid all other phenomena but absorption.

\subsubsection{Range of concentrations}

We assume that or device will only be able to perform absorbance measurements in a certain range of concentrations for a given fluid. This range will be delimited below by the limit of detection [3] of the device (which may change with the different layouts and different amounts of mirrors) and above by saturation of the device or the non-linearity of BeerLambert's law (which varies depending on the absorbing molecule and the experimental conditions) [4].

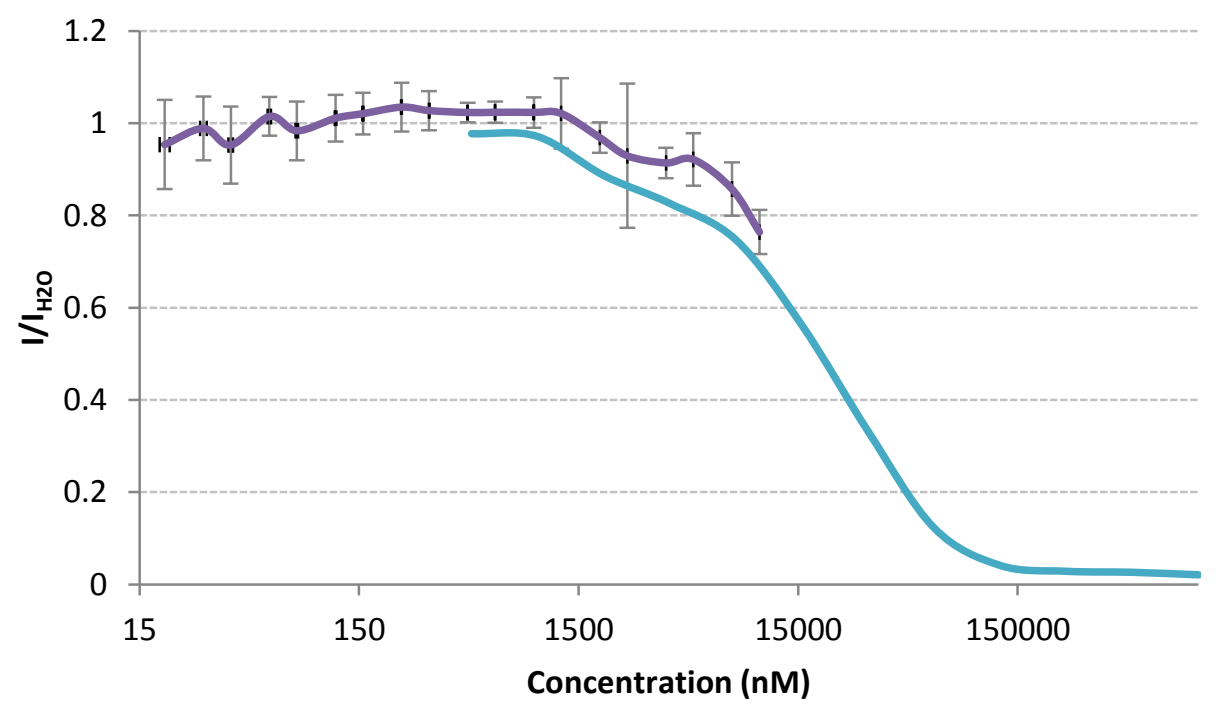

Fig. 5.2 Curves of the absorbed light intensity (quotient of intensity received with a certain fluorescein concentration over the intensity received with water) for a range of concentrations from $20 \mathrm{~nm}$ to $10000 \mathrm{~nm}$ (purple line) and from $400 \mathrm{~nm}$ to $1 \mathrm{mM}$ (blue line).

That is why we ran some previous absorbance experiments to give us an approach of what would be a valid concentration range to operate with fluorescein. We measured the absorption of fluorescein dilutions in water from $20 \mathrm{~nm}$ to $1 \mathrm{mM}$. This was made in two 
different sets of experiments: one from $20 \mathrm{~nm}$ to $10000 \mathrm{~nm}$ and the other one from $480 \mathrm{~nm}$ to $1 \mathrm{mM}$. The results of the absorbed intensity $\left(I_{\text {fluorescein }} / I_{\text {water }}\right)$ are plotted in Figure 5.2 in a logarithmic scale in order to check the linearity of absorbance -which is the logarithm of the absorbed intensity- with concentration.

From these results we can foresee that there is a range of concentrations for which the absorbance appears to be lineal. This gives us a clue that our microfluidic device operates properly and also allows us to make a good choice on our final concentrations to test the design of the chips in this chapter. To make a good characterization we want to go from the low flat region to high flat region in Figure 5.2, which means, from $250 \mathrm{nM}$ to $250000 \mathrm{nM}$ $(0.25 \mathrm{mM})$.

We used a series of fluorescein concentrations using as a starting point the $1 \mathrm{mM}$ fluorescein dilution in water (used previously for the ray tracing test in Chapter 4, Section 4.3.1). From this concentration we started to decrease the value in $1 / 2$ ratios, which is an easy way to go down with simple calculations and short room for imprecisions. The final series of concentrations used are shown in Figure 5.3.

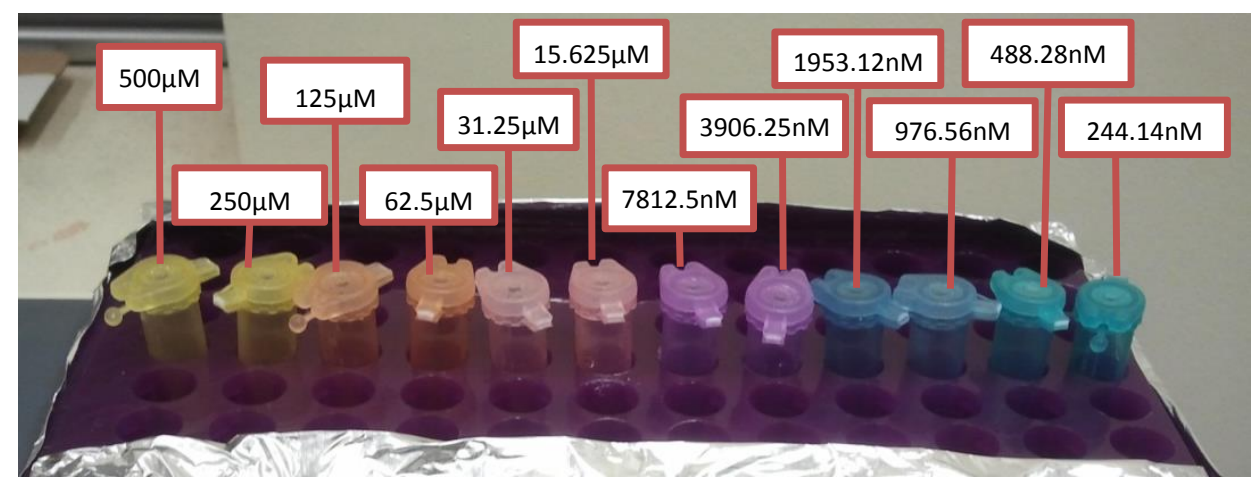

Fig. 5.3 Picture of all the aqueous fluorescein concentrations labeled, starting from $500 \mu \mathrm{M}$ and going down till $250 \mathrm{nM}$.

Now that we have a device, a fluid, a spectral band and a range of concentrations it is the time to make our definitive absorbance measurements to test our microfluidic device.

\subsection{Experimental results}

The instrumentation and setup of the following experiments was done as explained in the corresponding section of Chapter 3 (Section 3.3). For each concentration listed above, the average of 10 consecutive scans of the spectrometer was taken, using an integration time of $300 \mathrm{~ms}$, and then the intensity results for a wavelength interval between 200 and $1100 \mathrm{~nm}$ were stored in the computer and processed.

As it is said above, not all the spectrum will be useful for us, but only those wavelengths below $480 \mathrm{~nm}$. Figure 5.4.a shows the received intensity as a function of the wavelength for all the different dilutions used. It can be observed that above $500 \mathrm{~nm}$ all concentrations present almost the same light intensity level. This, as has already been explained, is because at those bands absorption pulls down and emission pushes up as concentration increases. Thus, one thing compensates the other resulting in very similar levels for all the different dilutions. 
A detail of the interest spectral band is displayed in Figure 5.4.b, showing how at those frequencies the received light intensity decreases as concentration -and thus, absorptionincreases.

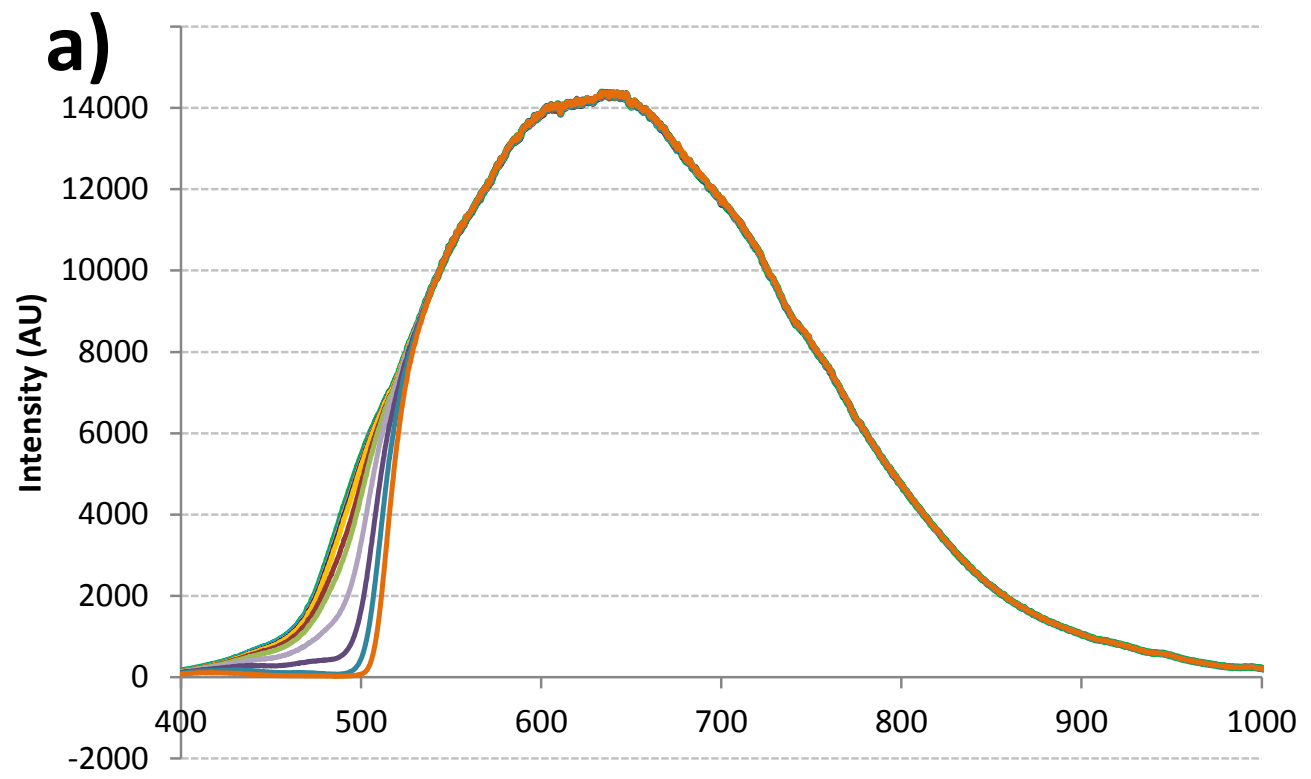

Wavelength (nm)
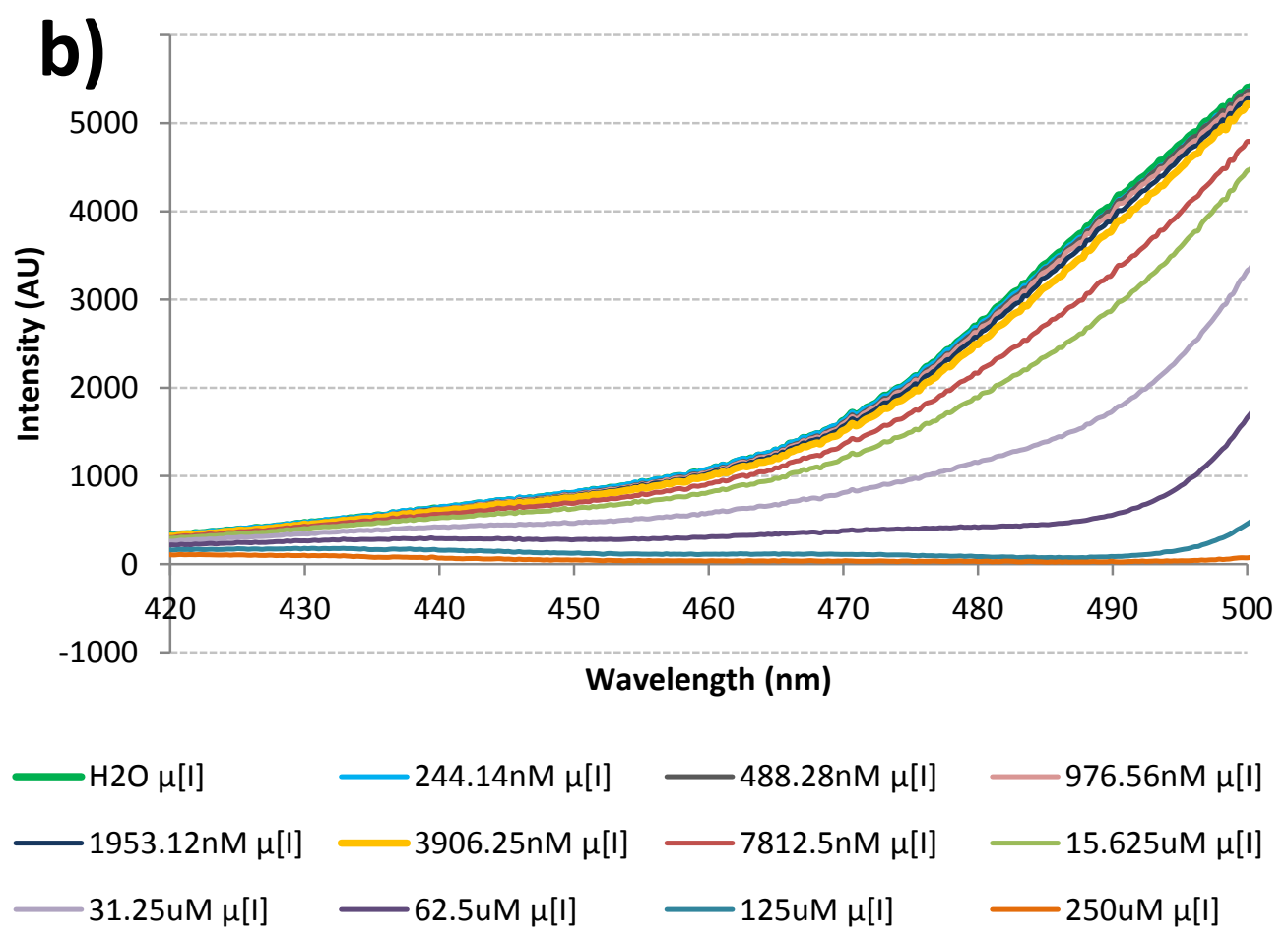

Fig. 5.4 Curves of the received light intensity as a function of the wavelength -through all the fluorescein dilutions- for (a) the whole spectrum covered by our lamp and (b) the interest band where no emission of fluorescein takes place.

We can see in Figure 5.4.a that as concentration increases, the received intensity decreases, and this means that there is more absorption, fitting correctly with what Beer-Lambert law 
says. However, this only gives us a qualitative approach that the theory is being followed, but in order to get a quantitative demonstration we need to plot the absorbance $\left(-\log \left[I_{\text {fluorescein }} / I_{\text {water }}\right]\right)$ as a function of the concentration and see if it follows a linear tendency. We do this for an intermediate wavelength of our operating range, namely, $450 \mathrm{~nm}$. The result is plotted in Figure 5.5.b.
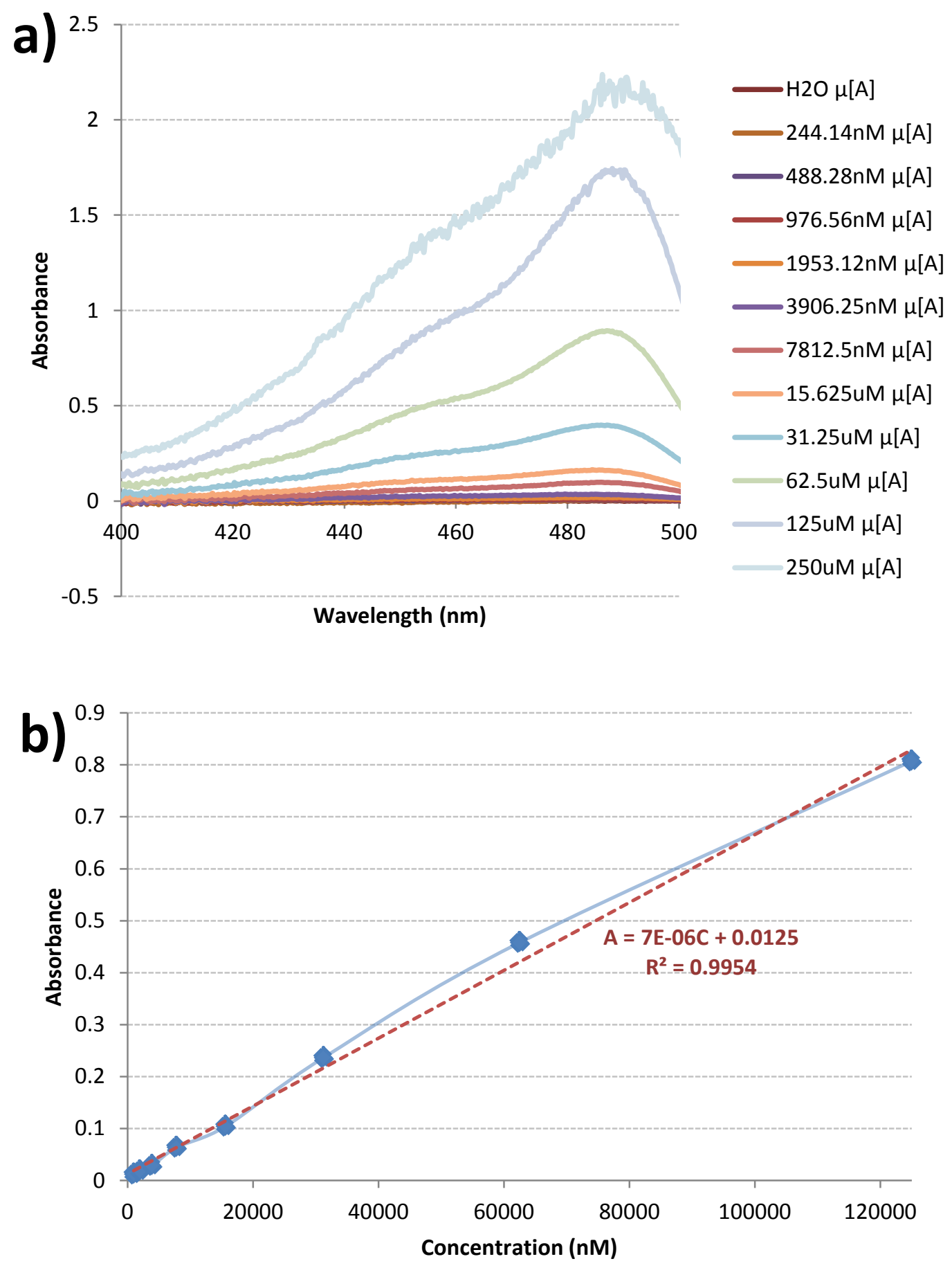

Fig. 5.5 (a) Absorbance as a function of the wavelength -note that at values near to $500 \mathrm{~nm}$ distortions due to emission start to take place and all the plots decay rapidly-. (b) Absorbance as a function of concentration for a wavelength of $450 \mathrm{~nm}$, proving the linearity according to BeerLambert's law and displaying the calculated trendline. 
Notice that the first two dilutions and the last one have been removed from the graphic of Figure 5.4.b. Remember that when we picked our concentration range we went from the nonlinear region below to the non-linear region above. The measurements of the samples corresponding to these regions are the ones that have not been included in the plot, since they would affect to the slope of the calculated trendline for the linear region. This slope $(\mathrm{m})$ will allow us to calculate the concentration of any given fluorescein dilution -as long as it belongs to the region of linearity- just with an absorbance reading:

$$
A=m C \Rightarrow \frac{A}{m}=C
$$

Eq. 5.1 Calculation of the concentration of a given sample by the absorbance reading and the slope of the experimental trendline.

Note that the slope ' $\mathrm{m}$ ' is indeed the product of the molar absorptivity of the analyte and the optical path length (Chapter 1, Equation 1.2). This means that the value of ' $\mathrm{m}$ ' is particular to the microfluidic device layout -i.e. the path length and number of mirrors- (Chapter 3, Section 3.1.3) and the fluid measured.

\subsection{Conclusions}

We performed successfully absorbance measurements and were able to prove that they followed the Beer-Lambert law -i.e. that concentration is proportional to absorbance- for the interval compressed between the limit of detection of the microfluidic device and the lineal limit of the law.

This makes our device capable to give measurements of concentration of fluids directly from an absorbance reading as long as the fluid has been properly characterized -i.e. that the linear slope for it has been calculated-.

Plus, if we superpose the measurements taken in this chapter (Section 5.2) with the ones taken previously to select an appropriate concentration range -i.e. the ones in the graphic of Figure 5.2- we see that they follow a very similar tendency (Figure 5.6). With this we prove the repeatability of the measurements taken with our microfluidic device, since these three sets were recorded in different days and with different chips.

Further improvements will target to lower the limit of detection of the device, mainly by comparing experimental data from the different layouts in Chapter 3 (Section 3.1.3) and finding out the optimal number of mirrors and optical path length. 


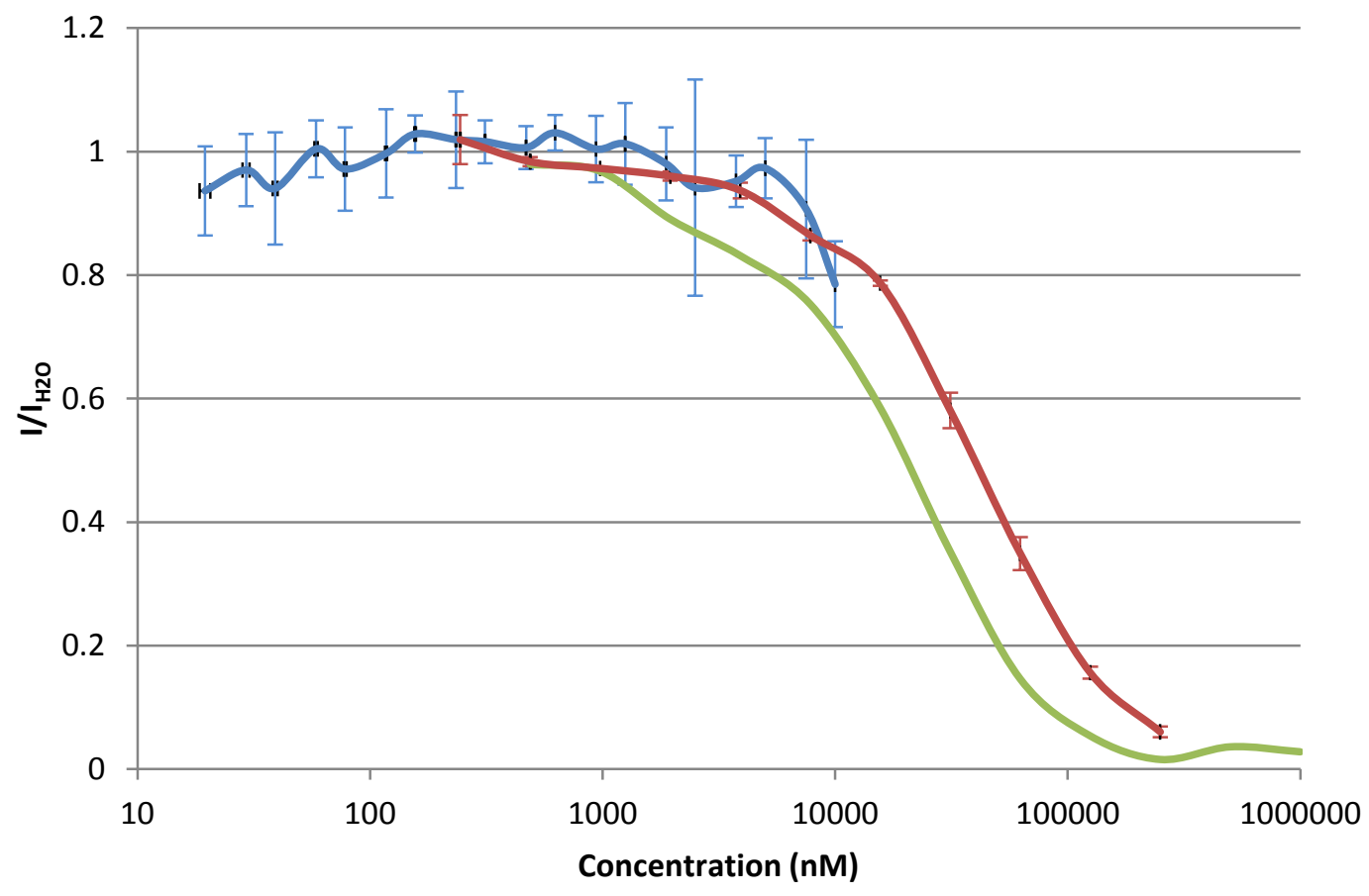

Fig. 5.6 Three plots of the absorbed intensity as a function of the concentration for a wavelength of $450 \mathrm{~nm}$ from three different sets of measurements taken with the microfluidic device in different days. The blue line corresponds to measurements with concentrations under the limit of detection; the green line, to concentrations a bit over the linear region for Beer-Lambert law; and the red line corresponds to the optimal range of concentrations.

\subsection{References}

[1] OceanOptics. HL-2000 Tungsten Halogen Light Sources [Online]. Available: http://www.oceanoptics.com/products/hl2000.asp

[2] Life-Technologies. Fluorescence SpectraViewer [Online]. Available: http://www.invitrogen.com/site/us/en/home/Products-andServices/Applications/Cell-Analysis/Labeling-Chemistry/FluorescenceSpectraViewer.html

[3] V. Thomsen, D. Schatzlein, and D. Mercuro, "Limits of Detection in Spectroscopy," Spectroscopy, vol. 18, pp. 112-114, 2003.

[4] A. Mehta. (2012). Ultraviolet-Visible (UV-Vis) Spectroscopy - Limitations and Deviations of Beer-Lambert Law [Online]. Available: http://pharmaxchange.info/press/2012/05/ultraviolet-visible-uv-vis-spectroscopy\%E2\%80\%93-limitations-and-deviations-of-beer-lambert-law/ 


\section{Chapter 6: Conclusions and future work}

We successfully developed a microfluidic PDMS device based in soft lithography that incorporates embedded metallic (indium) micromirrors. We did this following a monolithic approach and with a simple and inexpensive fabrication process. In this thesis, basically we characterize the implemented indium micromirrors and we prove that the device is able to perform absorbance measurements.

\subsection{Conclusions on indium micromirrors}

The calculated value of the reflectivity ( $R$ ) for a PDMS-Indium multilayer interface was around $85 \%$ for all the incident angles and wavelengths from 300 to $700 \mathrm{~nm}$. The integrated indium micromirrors were tested and the measured $\mathrm{R}$ was at least over a $40 \%$. This slight difference respect the calculated value is not necessarily due to a lack of quality in the fabricated mirrors, but also part of it can be because the light beam was wider than the mirror itself, so some light was escaping from our system.

Thus it is not the mirror that needs to be fixed but our focusing system. This can be solved just with a lens able to focus the light more precisely into the indium region. Future work to improve the reflectivity of the system will go through running simulations and design a better focusing lens at the input and output fiber ends, so all the light is directed and focused to hit the indium micromirrors.

\subsection{Conclusions on absorbance measurements}

The device was able to perform absorbance measurements properly and we could prove that it verified the Beer-Lambert's law with notorious precision for a determinate range of concentrations. This range depends on the fluid used and also on the layout of the microfluidic chip we are working with. It is delimited below by the limit of detection of the device (which depends on the optical path length and thus, on the amount of mirrors in the layout) and above by the non-linear region of Beer-Lambert's law (which depends mainly on the absorbing fluid).

Future work on characterizing this microfluidic device for absorbance measurements will focus on find out an optimal layout that lets us reach the lowest possible limit of detection and the most compact realization of the device. This will be done by adjusting the number of mirrors implemented and the angle of incidence of light. Note that there is a trade-off between this two variables, since lower angles result in shortening the path length since the fluidic channel is crossed less obliquely by the light. 


\section{Acknowledgements}

This thesis would not have been possible without the help and backup of some people. This section is dedicated to give them a space in this publication and express my gratitude to them.

In the first place, my gratitude goes to Dr. Raquel Perez-Castillejos, my project advisor. Thank you for accepting me in your lab, for the opportunity given, for all the advice and guidance and for all the work that you also put in this thesis.

Secondly I am really thankful to Raul Broto Cervera. He is the one who went through all the design of the device that was the object of study of this thesis and is consequently referenced in several chapters. However, he also shared spaces with me in the same lab for a couple of months; he made me a participant of his work, presented me the chips I was going to study on and taught me how to use the instrumentation in the lab.

I also want to express my gratitude to Steven Manole, mechanical engineering student in NJIT and a good friend of mine, who spent long hours of discussion with me when I was stuck on my work and gave me the final push that allowed me to fully characterize the indium micromirrors. Thank you for all the input and all the ideas you gave me, and all the whiteboards we filled together.

I am also very thankful to New Jersey Institute of Technology (NJIT) and Universitat Politècnica de Catalunya (UPC) for the opportunity given to me and the facilities that I have been disposing with to perform all my research.

And finally, I want to express my gratitude to my family, without whose moral and economic support would not have been possible this work. I also want to make special mention of the encouragement and moral back up that my girlfriend Anna always gave to me. 